\title{
Construction, assembly and tests of the ATLAS electromagnetic barrel calorimeter
}

\author{
ATLAS Electromagnetic Barrel Liquid Argon Calorimeter \\ Group*
}

\begin{abstract}
The construction and assembly of the two half barrels of the ATLAS central electromagnetic calorimeter and their insertion into the barrel cryostat are described. The results of the qualification tests of the calorimeter before installation in the LHC ATLAS pit are given.
\end{abstract}

(submitted to Nucl. Instrum. Methods A)

*) See next pages for the list of authors 
B. Aubert, B. Beaugiraud, J. Colas, P. Delebecque,

L. Di Ciaccio, M. El Kacimi ${ }^{a, b, c}$, P. Ghez, C. Girard

M. Gouanère, D. Goujdami ${ }^{a, b, c}$, A. Jeremie, S. Jézéquel, R. Lafaye, N. Massol, P. Perrodo, H. Przysiezniak, G. Sauvage ${ }^{1}$

J. Thion, I. Wingerter-Seez, R. Zitoun, Y. Zolnierowski

Laboratoire de Physique de Particules (LAPP), IN2P3-CNRS, F-74941 Annecy-le-Vieux Cedex, France ${ }^{b, c}$.

R. Alforque, H. Chen, J. Farrell, H. Gordon, R. Grandinetti, R. Hackenburg, A. Hoffmann, J. Kierstead, J. Koehler, F. Lanni, D. Lissauer, H. Ma, D. Makowiecki, T. Muller, S. Norton, V. Radeka, D. Rahm, M. Rehak, S. Rajagopalan, S. Rescia, K. Sexton, J. Sondericker, I. Stumer, H. Takai Brookhaven National Laboratory (BNL) Upton, Ny 11973-5000, USA.

A. Belymam, D. Benchekroun, C. Driouichi, A. Hoummada, M. Hakimi

Faculté des Sciences Aïn Chock,Casablanca, Morocco ${ }^{b, d}$.

M. Knee, R. Stroynowski, B. Wakeland

Southern Methodist University, Dallas, Texas 75275-0175, USA.

V. Datskov, V. Drobin

Joint Institute for Nuclear Research, Dubna.

M. Aleksa, J. Bremer, T. Carli, M. Chalifour ${ }^{e}$, J.L. Chevalley, F. Djama ${ }^{f}$, L. Ema, C. Fabre, P. Fassnacht, F. Gianotti, A. Gonidec, J.B. Hansen ${ }^{g}$,

L. Hervas, T. Hott, C. Lacaste, C.P. Marin, P. Pailler, A. Pleskatch ${ }^{h}$, D. Sauvaget, G. Vandoni, V. Vuillemin, H. Wilkens

European Laboratory for Particle Physics (CERN), CH-1211 Geneva 23, Switzerland. 
S. Albrand, B. Belhorma, J. Collot, P. de Saintignon, D. Dzahini, A. Ferrari, J. Fulachier, M.L. Gallin-Martel, J.Y. Hostachy, G Laborie, F Ledroit-Guillon, P. Martin, J.F. Muraz, F. Ohlsson-Malek, S. Saboumazrag, S. Viret

Laboratoire de Physique Subatomique et de Cosmologie (LPSC), Université Joseph Fourier, IN2P3-CNRS, F-38026 Grenoble, France ${ }^{b, d}$.

R. Othegraven, C. Zeitnitz

Johannes Gutenberg-Universität Mainz, Institut für Physik, D-55099 Mainz, Germany.

D. Banfi, L. Carminati, D. Cavalli, M. Citterio, G. Costa, M. Delmastro, M. Fanti, L. Mandelli, M. Mazzanti, F. Tartarelli

Dipartimento di Fisica dell'Università di Milano and INFN

I-20133 Milano, Italy.

E. Augé, S. Baffioni, J. Bonis, W. Bonivento ${ }^{i}$, C. Bourdarios, C. De la Taille, L. Fayard, D. Fournier, G. Guilhem, P. Imbert, L. Iconomidou-Fayard, G. Le Meur, M. Mencik, J-M. Noppe, G. Parrour, P. Puzo, D. Rousseau, A-C. Schaffer, N. Seguin-Moreau, L. Serin, G. Unal, J-J. Veillet, F. Wicek, D. Zerwas

Laboratoire de l'Accélérateur Linéaire, Université de Paris-Sud, IN2P3-CNRS, F-91898 Orsay Cedex, France.

F. Astesan, W. Bertoli, B. Canton, F. Fleuret, D. Imbault, D. Lacour, B. Laforge, Ph. Schwemling ${ }^{1}$ Laboratoire de Physique Nucléaire et de Hautes Energies, Université Paris VI et VII, IN2P3-CNRS, F-Paris, France.

E.M. Abouelouafa, A. Ben Mansour, R. Cherkaoui, Y. El Mouahhidi, H. Ghazlane, A. Idrissi

Faculté des Sciences and Centre National de l'Energie, des Sciences et Techniques Nucléaires, Rabat, Morocco ${ }^{b, d}$. 
K. Bazizi, D. England, V. Glebov, T. Haelent, F. Lobkowicz $\dagger$, P. Slattery

University of Rochester, River Campus, B\&LL Building, Rochester, New York 14627-0171, USA.

J. Belorgey, N. Besson, M. Boonekamp, D. Durand,

J. Ernwein, B. Mansoulié, F. Molinié, J.P. Meyer,

P. Perrin, J. Schwindling, J.P. Taguet, H. Zaccone

DAPNIA, CEA Saclay, F-91191 Gif-sur-Yvette Cedex, France.

B. Lund-Jensen, S. Rydström, Y. Tayalati

Royal Institute of Technology, Stockholm, Sweden ${ }^{b}$.

B. Botchev, G. Finocchiaro, J. Hoffman, R.L. McCarthy, M. Rijssenbeek, J. Steffens, M. Zdrazil

State University of New York, Stony Brook, New York 11794, USA.

\author{
H.M. Braun \\ University of Wuppertal, Wuppertal, Germany.
}

(a) visitor from LPHEA, FSSM-Marrakech (Morocco).

(b) partly supported by the "Calorimétrie électromagnétique à argon liquide d'ATLAS" GDRI between IN2P3/CNRS, the universities Joseph Fourier of Grenoble, of Méditerranée of Aix-Marseille II, and of Savoie, the Moroccan

CNRST and KTH Sweden.

(c) partly supported by the PAI MA/02/38.

(d) partly supported by the PAI MA/01/05.

(e) also CEA-Saclay.

(f) on leave from CPPM, Marseille.

(g) now at Niels Bohr Institute, Copenhagen.

(h) on leave from IFVE, Russia.

(i) now at INFN-Cagliari.

$\dagger$ Deceased. 


\section{Introduction}

The electromagnetic barrel calorimeter of the ATLAS experiment at CERN's future proton-proton collider, the LHC, is a lead-liquid argon sampling calorimeter with accordion shaped absorbers and electrodes. Calorimetry will be a crucial tool for the understanding of proton-proton collisions at the LHC, since many physics processes will manifest themselves through final states with electrons or photons: $\mathrm{W}$ and $\mathrm{Z}$ production, $H \rightarrow \gamma \gamma, H \rightarrow 4 e, H \rightarrow W W$. Precise measurements of the properties of the electrons and photons is of utmost importance, and dynamic range, resolution and uniformity are the main parameters to be optimized. From physics simulations[1], it has been determined that the electromagnetic calorimeter should meet the following requirements:

- For a Higgs boson decaying to two photons or to four electrons, in the mass range from 90 to $180 \mathrm{GeV} / c^{2}$, ATLAS should measure the Higgs mass with $1 \%$ precision using the calorimeter system alone. This translates into the requirements of a sampling term of less than $10 \% / \sqrt{E}$, associated with a constant term better than $1 \%$.

- The calorimeter must be able to separate the two photons from a $\pi^{0}$ decay up to transverse energies of $60 \mathrm{GeV}$.

- The dynamic range has to cover $30 \mathrm{MeV}$ up to $1 \mathrm{TeV}$, i.e. from the typical noise level in one single cell up to the single cell energy deposition expected in the case of the decay of a heavy $Z^{\prime}$ or $W^{\prime}$ with masses up to $6 \mathrm{TeV} / \mathrm{c}^{2}$.

Liquid argon calorimetry has been chosen for ATLAS because of its intrinsic linear behaviour, stability of the response over time and radiation tolerance. The accordion geometry has been chosen because it allows a very high granularity and a very good hermeticity, since the high voltage and signal cables run only at the front and back faces of the detector. In addition, the accordion geometry minimizes inductances in the signal paths, allowing the use of the fast shaping needed for operation with $25 \mathrm{~ns}$ bunch intervals between collisions at LHC.

The first studies of liquid argon calorimetry for LHC date back to 1990[2-5]. A "2 meter" long prototype was built by the R\&D collaboration RD-3, to evaluate the performances of liquid argon calorimetry for a LHC experiment. After the choice of the liquid argon technology by the ATLAS collaboration for its electromagnetic calorimetry in 1995, the geometry of the detector and

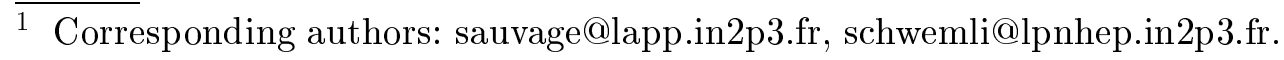


the design of its elements (absorbers, electrodes, G10 bars, rings, etc.) were reassessed in view of assembly line production, using the experience gained with the 1992 prototype. The assembly line production concept was validated by the construction in 1998 and extensive testing of a second prototype, called Module 0, using final or close to final series elements for its construction. The fabrication of some of the elements of the calorimeter, like the absorbers, started in the beginning of 2000, and the stacking of the modules took place between 2001 and mid-2003. During the stacking, four modules were studied in beam tests.

The paper is organized as follows. In Section 2, a general description of the barrel calorimeter is given and the construction of the main elements necessary to assemble a barrel module is described. The stacking of the barrel modules is the subject of section 3. The cold electronics and the cabling of a module are described in section 4 . The different monitors associated with the calorimeter are detailed in section 5. A description of the barrel cryostat and associated feedthroughs is given in section 6. The assembly of 16 modules in a half barrel is described in section 7 . The insertion of the two half barrels in the barrel cryostat and the qualification tests performed at room and liquid argon temperature on the entire calorimeter are the subject of section 8 . The main results of the calibration of the 4 modules tested with an electron beam are summarized in section 9. Finally, the conclusions are given in section 10.

\section{Calorimeter description}

The ATLAS electromagnetic barrel calorimeter, shown in Fig. 1 and described in detail in [6], is made of two half barrels, centered around the z-axis (ATLAS beam axis). One half barrel covers $z>0$ (pseudorapidity $\eta>0$ ) and the other one $z<0(\eta<0)$, from $|\eta|=0$ to $|\eta|=1.475$; the length of each half barrel is $3.2 \mathrm{~m}$, the inner and outer diameters are $2.8 \mathrm{~m}$ and $4 \mathrm{~m}$ respectively. The calorimeter is located behind the superconducting solenoid, in the same cryostat and weighs 114 ton. Since the material in front of the calorimeter amounts to about $1.5 X_{0}$ on average, the barrel calorimeter is complemented with a liquid argon presampler detector placed in front of its inner surface, over the full $\eta$ range.

A half barrel is made of 1024 accordion shaped absorbers, interleaved with readout electrodes. The electrodes are kept in the middle of the gap by spacers. The drift gap on each side of the electrode is $2.1 \mathrm{~mm}$, which corresponds to a total drift time of about $450 \mathrm{~ns}$ for an operating voltage of $2000 \mathrm{~V}$.

Once assembled, there is no discontinuity along the azimuthal angle $\phi$; but for ease of construction, a half barrel is divided into 16 modules. The total 
thickness of a module is at least 22 radiation lengths $\left(\mathrm{X}_{0}\right)$, increasing from $22 \mathrm{X}_{0}$ to $30 \mathrm{X}_{0}$ between $|\eta|=0$ and $|\eta|=0.8$, and from $24 \mathrm{X}_{0}$ to $33 \mathrm{X}_{0}$ between $|\eta|=0.8$ and $|\eta|=1.3$.

A module (Fig. 2) has three compartments in depth (front, middle, back). The readout granularity of the different compartments is shown in Table 1 for $\eta<1.3$. In total there are 3424 readout cells per module, including the presampler cells.

Table 1

Readout segmentation and depth (in $\mathrm{X}_{0}$ ) of the compartments of a barrel module and its associated presampler sectors for $\eta<1.3$.

\begin{tabular}{|l|c|c|c|}
\hline Compartment & $\Delta \eta$ & $\Delta \phi$ & $\mathrm{X}_{0}$ \\
\hline Presampler & 0.025 & $2 \pi / 64$ & 0.08 to 0.15 \\
Front & $0.025 / 8$ & $2 \pi / 64$ & 2.5 to 4.5 \\
Middle & 0.025 & $2 \pi / 256$ & 16.5 to 19 \\
Back & 0.050 & $2 \pi / 256$ & 1.4 to 7 \\
\hline
\end{tabular}

\subsection{Module components}

The main components of a module are listed below:

- Absorbers: the absorbers (Fig. 2 and 3) are made of lead plates (thickness $1.53 \mathrm{~mm}$ for $\eta<0.8,1.13 \mathrm{~mm}$ for $\eta>0.8$ ), glued between two $0.2 \mathrm{~mm}$ thick stainless steel sheets by resin-impregnated glass fiber fabric. The two lead thicknesses have been chosen to ensure a depth of at least $22 \mathrm{X}_{0}{ }^{2}$ for the calorimeter. The decrease in lead thickness after $\eta=0.8$ limits the decrease of the sampling fraction at high $\eta$. The fiber fabric compensates for the difference in thickness of the two types of lead plates so that the nominal thickness of an absorber is $2.2 \mathrm{~mm}$. The stainless steel sheets provide mechanical strength to the absorber.

- G10 precision bars: at the inner and outer edges, each absorber is encased into the groove of a precision machined G10 fiberglass-epoxy composite bar. The purpose of these bars (Fig.4) is to position each absorber with respect to its neighbours, and also to provide space for the connectors of the electrodes. The ultimate geometrical accuracy of the assembled cylinders is a direct consequence of the precise machining of the azimuthal contact surfaces of the bars.

$\overline{2}$ Including the material in front, the minimal depth is $24 \mathrm{X}_{0}$. 
- Outer and inner rings: 7 stainless steel outer rings support and give rigidity to a half barrel. Each ring (Fig. 5) is made of 16 ring-pieces corresponding to the 16 modules. Similarly, 8 composite inner rings help to define the inner geometry of a half barrel. Each inner ring (Fig. 6) is also made of 16 ring-pieces.

- Electrodes: the readout electrodes (Fig. 7) consist of three conductive copper layers separated by insulating polyimide sheets. The two outer layers are at the high voltage potential; the inner one is used for reading out the signal through capacitive coupling. Each gap between two absorbers is equipped with two electrodes: type A $(\eta<0.8)$ and type B $(\eta>0.8)$.

- Spacers: the spacers keep the electrodes centered between two consecutive absorbers. Each spacer (Fig. 8) consists of strips of honeycomb laid on the flat sections of the accordion absorber.

\subsection{Presampler}

The presampler is needed to correct for the energy lost upstream of the calorimeter, especially at low energy. For this purpose a separate liquid argon presampler, providing shower sampling in a thin liquid argon layer (11 $\mathrm{mm}$ in depth), is placed in front of the electromagnetic calorimeter inside the common barrel cryostat. It is made of 64 identical azimuthal sectors (i.e. 32 for a half barrel). Each sector is $3.1 \mathrm{~m}$ long and $0.28 \mathrm{~m}$ wide thus covering the half barrel length and giving a coverage in $\eta$ and $\phi$ of 1.52 and 0.2 respectively. It is composed of eight different sized modules with a length increasing with $\eta$ to obtain the same $\eta$ coverage of 0.2 for each module except the one at the end of the barrel whose $\eta$ coverage is reduced to 0.12 . The eight modules of a sector are housed in an external envelope which consists of a $0.4 \mathrm{~mm}$ thin glass-epoxy shell. Details of the contruction can be found in ref.[7].

The modules are made of interleaved cathode and anode electrodes glued between $\mathrm{FR} 4^{3}$ plates. The electrode spacing slightly varies with presampler module type from 1.9 and $2.0 \mathrm{~mm}$. The cathodes are $(270 \pm 30) \mu \mathrm{m}$ thick, double-sided printed circuit boards while the $\left(330 \pm_{40}^{30}\right) \mu \mathrm{m}$ thick anodes have 3 conductive layers separated by nominally 150 and $130 \mu \mathrm{m}$ FR4 layers. The required granularity $(\Delta \eta=0.025, \Delta \phi=2 \pi / 64)$ for each module is obtained by putting together the appropriate number of anodes in the $\eta$ (or $z$ ) direction and by subdividing (i.e. etching) each anode into two halves in the $\phi$-direction. $\mathrm{A}+2 \mathrm{kV}$ high voltage potential is applied to the outer layers of the anodes and the signal is readout through capacitive coupling to the central layer at ground potential. Each anode has four $1 \mathrm{M} \Omega$ surface-mounted resistors to feed the high voltage. Quality assurance tests of the anodes prior to module

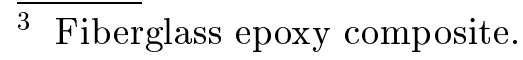


production include a $>24$ hour $\mathrm{HV}$ test at $3 \mathrm{kV}$, a measurement of the leakage current at this voltage and a RC-measurement to verify the connectivity of the different layers and the soldered resistors. The anode production and quality is documented separately [8]. Fig. 9 shows a presampler module.

A mother board which is a five layers printed circuit is mounted on each module. The mother boards, which come in eight different sizes to match the modules, collect signals from the readout cells. In addition the mother boards are equipped with a set of accurate surface-mounted resistors $(0.1 \%$ and $25 \mathrm{ppm} /{ }^{\circ} \mathrm{C}$ ) for the injection of calibration pulses.

The same type of HV, calibration and signal cables (see subsection 4.2.1) as for the calorimeter are used. Two separate HV lines per pair of modules, connected to the two sides of the anodes, are used to prevent the complete loss of a module in case of short-circuits.

Two assembly sites were used, each equipped with a station for testing sectors in liquid nitrogen. Each sector was first measured at room temperature with a test bench where the response to a low frequency, $10 \mathrm{kHz}$, sinusoidal signal injected through each of the HV cables was registered (called TBF test later). To eliminate deviations due to different read out amplifiers being used for different signal channels, the response was also measured when the same sinusoidal signal was injected through the calibration cables. This allowed the verification of the connectivity of all cables and anodes, including the 4 resistors on each anode. The test also included registering the response to a triangular test pulse injected through the calibration cables. The same tests were then repeated at liquid nitrogen temperature. Any failed connectivity was, if possible, repaired and the cold test repeated. Prior to the cold test, a voltage of $1 \mathrm{kV}$ was applied to the anodes, for at least one night. Finally each sector was tested at $2 \mathrm{kV}$ in liquid nitrogen for at least 24 hours.

A total of 66 sectors, including 2 spares, have been produced and validated. The validated sectors were stored in dry nitrogen gas inside a box made of an anodized extruded aluminium profile into which the sector could slide. This limited the humidity until installation on the barrel calorimeter.

\subsection{Absorbers}

\subsubsection{Lead sheet production}

Thickness variations of the lead plates used to build the absorbers induce local variations of the calorimeter response, which contribute to the constant term. Typically, an increase of $1 \%$ of the lead plate thickness leads to a $0.5 \%$ decrease of the measured signal. 
The flat lead sheets (1.53 and $1.13 \mathrm{~mm}$ thick) were produced by a rolling mill in industry. The thickness tolerance that the manufacturer was ready to guarantee, was $\pm 30 \mu \mathrm{m}$ (rms of $17 \mu \mathrm{m}$ ). This would translate into a contribution of about $1 \%$ to the constant term in the worst case, when neighbouring absorbers had correlated thickness fluctuations. To avoid such contributions to the constant term, two steps were taken:

- An X-ray measurement system was set up and used during the rolling operation to help the manufacturer in reducing the dispersion of lead thicknesses within the allowed tolerance interval. With this real time monitoring of the thickness, the operator could react very fast to drifts observed in the sheet thickness. In addition, the measurement system provided the possibility to reject out-of-tolerance lead. In this way, the lead sheets delivered to the collaboration had a thickness r.m.s variation of $6.6 \mu \mathrm{m}$.

- An ultrasound measurement system, with an accuracy of a few $\mu \mathrm{m}$ was used to produce detailed thickness maps (on a $5 \times 5 \mathrm{~cm}^{2}$ grid) for each lead plate. Using the fact that a typical shower develops, on average, in five consecutive absorbers, the detailed thickness maps of the plates have been used to optimize the arrangement of consecutive absorbers, by ensuring that neighbouring absorbers have opposite thickness fluctuations. It was established [9] that the constant term could be estimated from the r.m.s. variation of the normalized thickness of the lead plates, averaged over 5 consecutive plates. From the thickness measurements, it can be estimated that the residual contribution to the global constant term arising from the lead thickness fluctuations has been reduced to $0.19 \%$ (Fig. 10).

\subsubsection{Absorber fabrication}

First, a flat sandwich of stainless steel, resin-impregnated glass fiber fabric and lead plates was assembled from the separate parts. Then the flat sandwich was bent to an accordion shape, using a bending machine with the following principle: the upper and lower bending knives of the machine were positioned with a precision of $0.1 \mathrm{~mm}$ with respect to each other and to the central top knife, which was fixed. The other knives could be freed to move laterally. The sandwich first got pinched between the two sets of knives. At this stage, the knives were freed, and the upper knives were pushed down by the press, while simultaneously all the knives moved laterally towards the fixed knife in the center. The driving force was the friction between the surface of the sandwich and the tips of the knives. After bending, the sandwich was cured at $125^{\circ} \mathrm{C}$ in a heating press which consisted of two single-piece steel matrices machined with a $0.02 \mathrm{~mm}$ tolerance with respect to the absorber shape at $125^{\circ} \mathrm{C}$. This heating press applied a programmable pressure on the finished sandwich, up to 7 bars, using mechanical jacks, and followed a computer-controlled curing cycle. Since the final shape of the absorber was defined by the parameters 
of the heating press rather than the bending press, it had been chosen to use only one heating press (Fig. 11) to produce all the absorbers, to ensure the best possible reproducibility of the finished absorber shape. After curing and cooling down to room temperature, the G10 bars were glued to each absorber, using rotating stands, that allowed the accordion to compress or extend slightly to bring it to its nominal span. The curing of the glue was done at room temperature and took one day.

Final control of the geometry of the absorber was done using a 3D coordinate measurement machine. The measured geometrical quantities were : distance between bars, length of folds, thicknesses at several places, absorber width and angles between folds. Over the whole production period, typical measurements like distances between bars, absorber width, etc., whose stability was essential to ensure trouble-free stacking of the absorbers, have shown stability of the order of $0.1 \%$, whereas the stability of the absorber thickness ${ }^{4}$ (Fig. 12) measurement was at the level of $1 \%$. In a manner similar to the lead plates, it was possible to deduce from the fluctuations of the absorber averaged thickness, which induce correlated gap variations, a contribution to the constant term of $0.07 \%$ (Fig. 13). The absorber thickness has also shown a consistent pattern over time, with the middle of the absorbers being typically $40 \mu \mathrm{m}$ thicker than the extremities. The correlated gap variation induces a $0.29 \%$ maximal variation of the signal along $\eta$ direction which can be corrected for in the data analysis.

\subsection{G10 precision bars}

On both sides, at small (inner) and large (outer) radii, each absorber is glued, over its full length, to a G10 precision bar (Fig.4). A detailed drawing of these inner and outer bars can be found in [6]. These bars are azimuthally in contact with each other and define the exact cylindrical geometry of the whole calorimeter at warm temperature. Their role is multiple: they maintain the read-out electrodes at their correct $z$-position, they protect and hold the signal connectors, they fasten the embedded absorbers radially ensuring a minimal sagitta under gravity.

The material of the bars is fiberglass-epoxy, but with quite specific requirements on the temperature expansion coefficients. The external and internal bars must ideally have the same longitudinal contraction as the absorbers $\left(3 \times 10^{-3}\right.$, i.e. $3 \mathrm{~mm} / \mathrm{m}$ going from room temperature to liquid argon temperature). In addition, the external bars must have the same contraction coefficient in $\phi$ as the outer rings which are stainless steel $\left(2.5 \times 10^{-3}\right)$ whereas a smaller contraction coefficient for the inner bars is needed in order to keep them in

4 The nominal thickness is $2.21 \mathrm{~mm}$. 
contact when cold. This has been achieved by industry, producing large sheets of material made out of numerous tissue layers, each woven with symmetrically placed glass fibers. With an angle of $60^{\circ}$ between the two directions, values of $(3.01 \pm 0.05) \times 10^{-3}$ and $(2.25 \pm 0.10) \times 10^{-3}$ respectively for the longitudinal and the transverse coefficients, have been achieved. The thermal conductivity of the bars, important for the cool down and for the temperature uniformity of the calorimeter was measured to be $0.35 \mathrm{~W} \mathrm{~m}^{-1} \mathrm{~K}^{-1}$ at room temperature.

To get the best-possible geometrical accuracy of the cylinder, the thickness of the bars as well as the projective angles of both faces had to be rectified with high accuracy. After machining, the cumulative thickness of the $3 \mathrm{~m}$ long G10 precision bars was controlled for each batch of 16 bars. Fig. 14 shows, as an example, the distribution of 60 batches of 16 bars. A precision better than $0.02 \mathrm{~mm}$ for 16 bars (typically $5 \mu \mathrm{m}$ per bar) has been achieved. The bars are positioned in $z$ and radius with respect to each other by pins passing through precision holes drilled in the bars. A precision better than $0.01 \mathrm{~mm}$ in radius and $0.03 \mathrm{~mm}$ in $z$ has been achieved. The signal outputs of the read-out electrodes [12] pass through grooves machined radially in the bars. Cut-outs were machined on the outside faces (inner and outer radius of the detector) to fit the connectors. The two electrodes, type A and type B, were positioned by means of precision holes $(0.1 \mathrm{~mm}$ accuracy) drilled into the electrodes and the bars.

After a pre-production order to qualify the bars on the Module 0 [28] the final order for the full batch of 4096 bars was placed to industry. The manufacturing of the raw material, the rectangular raw bars preparation, the groove for gluing the absorber, the precise hole drilling and the machining of the notches and of the cut-outs were performed by industry. The grinding of both faces has been done at CERN with an in-house developed grinding machine. The production

of the raw material and machining of the bars has taken two years.

\subsection{Outer and inner rings}

\subsubsection{Outer rings}

The 1024 absorbers making one half barrel calorimeter are screwed onto seven non-magnetic stainless-steel ${ }^{5}$ rings [6]. The aim of these supporting rings is to give the necessary rigidity to the entire half barrel and to define precisely its geometry. These rings stand on the cryostat rails.

One ring is made of 16 ring-pieces, $22.5^{\circ}$ in $\phi$, a ring-piece corresponding to

5 INOX $316 \mathrm{LN}$. 
one module of 64 absorbers. The 16 ring-pieces are screwed together with $\mathrm{Cu}$-Be bolts to form a complete circle (the $\mathrm{Cu}$-Be alloy has a slightly higher thermal coefficient than stainless steel, such that the ring-pieces are even more tightened at cold). The 16 ring-pieces are identical and have a cross-sectional shape of an I-beam (Fig. 5) except for the two ring-pieces at the level of the cryostat rail (see section 6). The dimensions have been chosen so that the deformation under the weight of the calorimeter is tolerable, i.e. less than $4 \mathrm{~mm}$ (see Fig. 15).

The rings are spaced approximately 0.2 units of rapidity apart to ease the cabling of the electronics. The weights supported by the seven rings are in tons, respectively, 5.8, 6.7, 8.3, 9.1, 8.6, 9.1, and 9.4 from $\eta=0$ to $\eta=1.2$, the weight of a half barrel being 57 ton. The cross-sectional shape of the first ring, close to $\eta=0$, has been modified to give the necessary clearance for the middle flange of the cold outer wall of the barrel cryostat.

For the assembly phase, the inner radius of the ring was the reference radius on which the external G10 bars of the 1024 absorbers were mounted. Special care has been taken to get a reference radius as close as possible to its nominal value $\left({ }_{-0.1}^{+0.0} \mathrm{~mm}\right)$ in order to avoid any clearance between the external G10 bars.

To assemble a module, the ring-pieces of the 6 rings from $\eta=0.2$ to $\eta=1.2$ were fixed onto a module assembly backbone. The role of this backbone was to give rigidity to the ring-pieces, to give a reference in $\phi$ and to allow the mounting of a half barrel with 16 modules. In order to maintain the reference radius, the outer radius of the ring-piece and the inner radius of the backbone supporting part were machined to the same accuracy as the internal ring radius. To have a unique $\phi$ reference for the 16 modules of a half barrel, a blank assembly of the 16 backbones and of the $6 \eta \neq 0$ rings resting on them was performed. A dowel pin going through each ring-piece and the corresponding supporting part of each backbone maintained the reference in $\phi$ during stacking.

A special jig to measure the deformation and the stresses of a ring under the weight of the calorimeter has been built. The radial deformations under a load of 10 ton ( 5 ton) of the $12 \eta \neq 0$ rings ( $2 \eta=0$ rings) have been measured [11]. The maximal deformations were observed at the top (decreased radius) and at the bottom (increased radius) of the ring. The averaged values and the rms for the $12 \eta \neq 0$ rings were respectively $(-2.00 \pm 0.15) \mathrm{mm}$ and $(3.20 \pm 0.15) \mathrm{mm}$. The upper plot of Fig. 15 shows the radial deformation measured at the middle of each ring-piece of the prototype ring compared to the results of the finiteelement calculation. 


\subsubsection{Inner rings}

The internal rings [6] fulfil four functions. During the assembly phase they ensured the mechanical rigidity of the module at the internal radius level. At cold they provide a small tension of the absorbers in such a way that all absorbers are in extension, even those which are located at the bottom of the calorimeter. At cold, also, they index the absorbers so that the gaps appearing at cold are equally distributed between absorber G10 bars. Finally they support the presampler rails.

The 8 rings of one half barrel are placed close to every $\eta=0.2$ to ease the cabling of the electronics. They are also made of 16 ring-pieces, $22.5^{\circ}$ in $\phi$. Bumps at the inner face allow the fixing of the presampler rails. These ringpieces (Fig. 6) are $40 \mathrm{~mm}$ wide and $10 \mathrm{~mm}$ thick, except at the level of the bumps where the thickness is $20 \mathrm{~mm}$. The ring close to $\eta=0$ has a width of $20 \mathrm{~mm}$ only. The space between the presampler and the rings, about $10 \mathrm{~mm}$ in radius, allows the passage of the electronic cables connecting the mother boards to the patch panel mounted at the end of the module. The gear-wheel shape of the ring at its outer face allows the indexing of the absorbers. Every second absorber is screwed on this internal ring. The ring-pieces are made of composite material. Two titanium dowel pins are inserted at both ends to allow the junction of two ring-pieces with titanium closing links.

The different thermal expansion coefficients of the stainless-steel external rings, of the absorbers, of the internal G10 bars and of the inner ring-pieces are such that a small gap $(0.024 \mathrm{~mm})$ appears at cold between two consecutive inner G10 bars. The gear-wheel shape of the inner ring at outer radius ensures a regular sharing of these gaps. Furthermore, the inner rings are in extension to avoid the compression of the absorbers located at the bottom of the calorimeter. To fulfil the preceding conditions, the inner ring-pieces are made of unidirectional G10. A special jig has been built to qualify each inner ring-piece at their nominal tension: 2.3 ton for $\eta>0$ ring-pieces and 0.8 ton for $\eta=0$ ring-pieces. The tolerance on the teeth of the ring-gear, $15 \mu \mathrm{m}$, has to allow the mounting of the module and has to be as small as possible, since this tolerance contributes directly to the gap variation.

\subsection{Electrodes}

The readout electrodes, $275 \mu \mathrm{m}$ thick, consist of three conductive copper layers separated by insulating polyimide sheets. The two outer layers are at the high voltage potential and the inner one is used for reading out the signal through capacitive coupling. The granularity of the calorimeter in $\eta$ and in depth is obtained by etched patterns on the different layers (Fig. 7). Each gap between 
two absorbers is equipped with two electrodes: type A $(|\eta|<0.8)$ and type B $(|\eta|>0.8)$.

The three longitudinal compartments have the following characteristics:

- The first compartment has been optimized for $\gamma / \pi^{0}$ identification. Its depth is around $6 X_{0}$, including about $1.6 X_{0}$ of dead material in front of the active part of the calorimeter. It is very fine grained in $\eta$, with a granularity of $\Delta \eta=0.025 / 8$.

- The second compartment, from 6 to $24 X_{0}$ has been optimized to contain photon showers up to $50 \mathrm{GeV}$. Its granularity is $\Delta \eta=0.025$.

- The third compartment has an additional $2 X_{0}$ or more and has a granularity of $\Delta \eta=0.05$. This compartment helps to reconstruct high energy showers and to discern between hadronic and electromagnetic showers.

Seven different types of electrodes would have been needed to cover the entire $\eta$ range of a half calorimeter, if the electrodes were to be within "industry standard" dimensions $\left(\simeq 100 \times 60 \mathrm{~cm}^{2}\right)$. To avoid difficulties with the precision mounting of the electrodes and to reduce the number of transition regions, we have chosen to develop and produce "large" electrodes of about $200 \times 100 \mathrm{~cm}^{2}$, to cover the calorimeter with only two electrode types.

The distribution of the high voltage to the outer layers of the electrodes is done by means of resistive silk-screened pads of carbon-loaded epoxy ink. The layout of the resistors and the properties of the ink have been defined with the following main constraints in mind:

- Avoid cross-talk between calorimeter cells in depth and protect the input of the preamplifiers in case of accidental discharges.

- Avoid more than one per mil voltage drop due to ion build up in the liquid argon at high luminosity. To meet these two opposite constraints, the resistivity of the ink has to be between 0.1 and $1 \mathrm{M} \Omega / \square$.

- Provide some redundancy in the high-voltage distribution, where possible.

Flat electrodes were produced in industry. The electrodes were firstly checked visually for misalignment between the layers, bad etching and gluing, silkscreening defects, mis-manipulation during fabrication. Then the resistance of all the resistive pads and the capacitance of all the cells were measured using automated test benches. These measurements were also used to search for short-circuits, open connections and other anomalies. Finally, each electrode had also to pass a high-voltage test.

Flat electrodes have to be bent to accordion shape. The first technique used implied bending the electrode bend-by-bend, with vertical knives activated one after the other to form folds at increasing radii, pushing the electrode into a mould made to the final shape of the bent electrode. However, the elasticity 
of the electrode material tends to increase the fold angles after bending. To compensate for this, the electrodes were cured a few hours in elastomer moulds at a temperature of $140^{\circ} \mathrm{C}$.

Later on, a different technique was applied. A press with a single knife was used. The knife was pressed against the electrode, with the electrode lying on a hard rubber base. In this way, the folds could be overbent, so that once the knife was removed, the required final shape was obtained without curing. It turned out that this second method was better adapted to a good bending than the first one.

After bending, electrodes were equipped with grounding springs, and the same electrical measurements as the ones performed on flat electrodes were redone, to check for any damage during bending, especially on the silk-screened resistors. Increases in the resistance value were indications that the resistor had started to crack. Such cracked resistors were repaired using silver ink. Finally, high voltage tests were again performed, including a long duration test at $2.5 \mathrm{kV}$, before shipping the electrodes to the three stacking sites. More details on the design, production and testing of the electrodes can be found in [12].

\subsection{Spacers}

The electrodes are kept centered in argon gap by the spacers. Nomex ${ }^{\circledR} 6$ paper impregnated with phenol-formaldehyde resin and with a honeycomb structure was produced and cut into sheets by Hexcel Composites (Belgium). The sheets, $(1.75 \pm 0.1) \mathrm{mm}$ thick, were then cut into strips, to be put on the flat parts of the accordion straight sections, on both sides of the electrodes. The honeycomb cells are $3 / 8$ inches wide for most strips, but we used honeycomb with $3 / 16$ inches wide cells for the strips in the first and last half folds of the accordion. The strips were assembled into accordion shaped sheets with the help of 14 glue impregnated glass-fiber threads, put on one side of the honeycomb (Fig. 8). A sheet is about half the module length, and two sheets are juxtaposed in $z$ in a half gap. To avoid damaging the electrodes, the thread is always put on the absorber side during stacking. Thus, two types of spacers ("A" and "B" types, with thread at the "top" or at the "bottom") were needed. The threads were put in place by the use of the tool shown in Fig. 16. To reflect the $0.16 \mathrm{~mm}$ opening of the gap along a fold, the tool had an opening gap geometry, and the thread was more or less pressed into the honeycomb. Four such tools were put together in an oven to polymerize the glue in the threads. This allowed four spacers (2 As and $2 \mathrm{Bs}$ ) to be built in one thermal cycle of 2 hours, leading to a typical rate of 16 spacers manufactured

${ }^{6}$ Nomex ${ }^{\circledR}$ is a registered trademark of DuPont Company. 
per day ${ }^{7}$. The thickness of one spacer out of ten was inspected on 28 spots on the threads. This allowed us to monitor small (around $0.1 \mathrm{~mm}$ ) drifts in the thickness and to readjust the tools. The averaged density of the spacer (or its transparency) is about $32 \mathrm{~kg} / \mathrm{m}^{3}$.

While stacking the first production module, high voltage problems were experienced, which could be attributed to surface contamination of the spacers. We thus decided to clean spacers individually in the laboratories by blowing dry nitrogen and by vacuum cleaning, and to test all of them at $3 \mathrm{kV}$ before stacking. In parallel, the fabrication in industry was transferred to a cleaner environment. The high voltage test was performed as follows: each spacer was put individually between two half-absorbers. The high-voltage was raised to $3 \mathrm{kV}$ and the spacer was given four chances to sustain this voltage during 3 minutes. About 90 to $95 \%$ of the spacers were accepted with this criteria and put in the modules. The remaining 5 to $10 \%$ were then cured by being held at $3.5 \mathrm{kV}$ for ten minutes. A large number of sparks was observed, but with no degradation of the spacer quality (no carbon deposit). After curing, the spacers underwent a new $3 \mathrm{kV}$ test, and most of them (95\%) were accepted. With these procedures, the number of gaps not holding $2 \mathrm{kV}$ which had to be restacked was down to a few per module.

\section{Module assembly}

\subsection{Cleaning}

Upon reception at the assembly site, the absorbers were thoroughly cleaned and stored in a clean area. Just before stacking, absorbers and electrodes were cleaned again. The spacer cleaning described in the preceding section was also repeated just before stacking. All the stacking took place in a class ISO 8 clean room, temperature controlled $\left((20 \pm 1)^{\circ} \mathrm{C}\right)$ and humidity controlled $(<$ $50 \%$ ).

\subsection{Stacking bench}

The stacking bench allowed for a relatively easy stacking, a precise mechanical assembly, and a number of mechanical and electrical controls. The bench base received the backbone onto which the modules were to be assembled. The bench could be rotated by $90^{\circ}$ around the longitudinal axis: in the horizontal

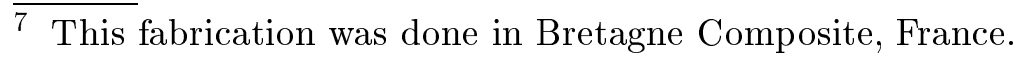


position one could present a new absorber (or a new spacer or electrode) and place it naturally on top of the previously stacked pieces. The vertical position was used to perform mechanical measurements and to tighten the fixation screws, so that the geometry of the module was minimally affected by the sagging of the absorbers and by the fact that the module was not yet complete. The control of the thickness of the stack is essential for the detector uniformity and for the final assembly of modules in a half barrel. Electrical tests were performed during stacking. The typical assembly rate was 4 gaps/day for each bench.

\subsection{Procedure}

The external ring pieces were first bolted to the backbone. Their position in $\phi$ was fixed by a hole-and-pin in the backbone and the ring piece as described above in section 2.5.1. Nevertheless a careful inspection and adjustment was performed before fixing $( \pm 0.2 \mathrm{~mm})$. The backbone was installed on the base of the bench, and adjusted in all directions $( \pm 0.2 \mathrm{~mm})$. The $z$ stop was adjusted, which defined the $z$ location of the absorbers. These adjustments were done with the help of precision jigs which gave the theoretical shape of a module. The first absorber was placed on the bench (horizontal), then the bottom spacers, then the electrodes A and B, then the top spacers; the elastic pins were put in place, then the second absorber. The fixation screws, between the absorber G10 bars and between the ring-pieces and the external G10 bars, were set in place. The bench was rotated to vertical. Two rows of pneumatic jacks pressed on the external and inner bars of the stack. The fixation screws were tightened at a controlled torque. The process was repeated 64 times. In addition in gap 32, 6 temperature probes were glued on the absorber. In the last gap, which would be later opened for the final half barrel assembly, the spacers were specially fixed.

\subsection{Mechanical and electrical controls during stacking}

The sagitta of the last stacked absorber was measured at $3 z$ positions. Every 4 absorbers, the thickness of the stack was measured at the inner and external bars, at 3 positions along $z$, using jigs as a reference $( \pm 0.02 \mathrm{~mm})$. If the stack was too thin by more than $0.05 \mathrm{~mm}$, the next gap was shimmed with $0.05 \mathrm{~mm}$ thick kapton pieces put on G10 bars.

Once a new gap was stacked, the high voltage was set to $2.2 \mathrm{kV}$ for a short duration and the connectivity was checked with the TBF test: a low frequency sinusoidal signal was fed to the HV lines and read out on the signal lines. The response was checked against a reference pattern. Every night, the HV was 
set to $2.2 \mathrm{kV}$ on the gaps stacked during the day (typically 4). The currents were recorded and checked in the morning. Each week-end a longer HV test at $2 \mathrm{kV}$ on the gaps stacked during the week (typically 16) took place. Once a module was fully stacked, a longer $\mathrm{HV}$ test at $2 \mathrm{kV}$ of at least 3 days on the 64 gaps of the module took place.

\subsection{Complete stack equipment and test}

Once the 64 gaps were stacked, the geometry of the module was measured. As there was still some elasticity of the inner radius thickness, it was fixed to the nominal value by external means. The inner rings were brought in. To ensure a precise location of the absorbers in the full detector, there must be no play between the indexing teeth of the inner rings and the absorber locations. Since the rings could not be machined with teeth to the needed precision, the teeth were first set in place separately, then the inner ring was set in place and glued to the teeth.

The gap capacitance was measured in each sector of $\Delta \eta=0.2$. The uniformity of gap thicknesses was checked (Fig. 17). Using the observed dispersions of the sliding average over 5 consecutive gaps, which were in the range $0.5 \%$ to $0.7 \%$ depending on the module, a global contribution to the constant term of $0.16 \%$ was estimated, using the 0.3 proportional factor between gap thickness variation and response variation[10]. Table 2 summarizes the different mechanical contributions to the constant term.

Table 2

Contributions to the constant term of the different mechanical non-uniformities

\begin{tabular}{|c|c|c|}
\hline lead thickness & absorber thickness & gap thickness \\
\hline $0.19 \%$ & $0.07 \%$ & $0.16 \%$ \\
\hline
\end{tabular}

\section{Cold electronics and cabling}

\subsection{Summing boards and mother boards}

The front section of the EM barrel calorimeter is read out at the inner radius, whereas the middle and back sections are read out from the back (outer radius). The readout is accomplished by several interconnected boards. These include summing boards that generate analog sums from adjacent calorimeter 
gaps and mother boards for analog sums readout and for calibration pulses distribution.

The summing boards are connected to the electrode connectors grouping the signals in $\phi$ to the desired readout granularity (Table 1). Each summing board services 16 electrodes $(\Delta \phi=0.1)$. In the front section all 16 electrodes are summed into one readout cell while the sums in the middle and back have finer granularity $(\Delta \phi=0.025$, or 4 adjacent electrodes). The boards are 10 layer PCBs with low-impedance $15 \Omega$ strip-lines whose lengths are equalized in $\phi$ in order to ensure a low, close to uniform, inductance of $25 \mathrm{nH}$ across all readout sections of a summing board.

A mother board provides readout for a $\Delta \eta \times \Delta \phi$ of $0.2 \times 0.2$ zone. It can cover anywhere between 4 summing boards (typically in the back section) to 16 summing boards (in the high rapidity region of the front section). Since each mother board reads out a constant rapidity region, the physical size of the mother board increases with rapidity. There are 7 different sizes varying in rapidity for the back section, and 8 for the front section, for a total of 14 back and 16 front mother boards for each barrel module.

The receptacles at the output of the summing board and the input of the mother board are connected via pins mounted on 2 thin PC-boards. Each channel is then connected twice. Each pin requires an insertion/exertion force of over 100 grams and there are over 100 pin connections between each mother board and summing board. For the outer radius, one out of every two PCboards also houses a low capacitance transient voltage suppressor (TVS) [13] with a turn-on at $6 \mathrm{~V}$ to protect against accidental discharges that may damage the calibration resistors mounted on the mother board. A thin G10 board electrically separates the summing board and the mother board.

The mother boards route the outputs to the readout cables through a "low profile" connector designed to minimize cross-talk between readout channels. The mother boards also include $0.1 \%$ at $90 \mathrm{~K}$ precision tantalum nitride calibration resistors (non-hygroscopic with a temperature coefficient of $70 \pm 5 \mathrm{ppm} /{ }^{\circ} \mathrm{C}$ ) to distribute calibration pulses to all readout channels. The value of the calibration resistor in various parts of the detector are chosen to match the expected currents from particle showers: the strip section uses $R_{\text {inj }}=3 \mathrm{k} \Omega$, the back section uses $R_{\text {inj }}=1 \mathrm{k} \Omega$ while the middle section uses $1 \mathrm{k} \Omega$ for $\eta<0.8$ and $500 \Omega$ at higher rapidities. The calibration pulse is injected at the ana$\log$ input to the mother board, the closest point to the origin of the analog signals from the electrodes. The length of the traces from the precision resistive network to the injection point are equalized for timing and terminated with $50 \Omega$ to avoid any reflections. The differences in the time-of-flight of particles coming from the vertex to different rapidity points in the calorimeter are compensated by adjusting the path lengths of the calibration signals on 
the mother board. This ensures that the relative timing between the trigger system and the signal are the same for calibration and for particles.

\subsection{Cables and connectors}

\subsubsection{Signal cables}

In order to cope with the $25 \mathrm{~ns}$ interval between bunch collisions of the LHC, a fast shaping of the calorimeter signals is necessary. For this purpose, the time constant of the $\mathrm{CR}-\mathrm{RC}^{2}$ shaping amplifier following the preamplifier on the same electronics board was chosen to be $15 \mathrm{~ns}$. At this frequency, the cable between the detector cell (a capacitance) and the preamplifier behaves mainly as a transmission line. This means, in particular that its capacitance does not contribute significantly to the noise, even for cable lengths of several meters. Taking advantage of this fact, it was decided, for better reliability, to put preamplifiers in the warm, in front-end crates located on cryostat feedthroughs.

A careful optimization of the cable and preamplifier, for the different detector cell capacitances and cable lengths (up to $4 \mathrm{~m}$ ) lead to the choice of two groups of cable impedance and preamplifier: $50 \Omega$ for the presampler and calorimeter strip section and $25 \Omega$ for the middle and back sections. Cables of $50 \Omega$ impedance are also used to carry calibration signals produced on dedicated boards, in the Front-End crates, down to the precision resistors located on the mother boards.

For practical matters, the choice was made to use mini coaxial cables, grouped in bundles of 64 , called harnesses hereafter, with a connection at the module end on patch panels. Harnesses A go from mother boards to patch panels, and harnesses $B$ from patch panels to cryostat feedthroughs. The feedthroughs themselves include short sections $(\simeq 25 \mathrm{~cm})$ of flat cables of $33 \Omega$ impedance (a compromise allowing a single type to be used for both the $25 \Omega$ and the $50 \Omega$ cables) which are described in subsection 6.2.

\subsubsection{Cable design}

The main constraints imposed on the cables were:

- Compatibility with liquid argon operation: mechanical flexibility at low temperature, no pollution of the liquid argon.

- Resistance to radiation: stable electrical performances, correct mechanical behaviour and absence of liquid pollution up to 10 MRads and $5 \times 10^{15} \mathrm{n} / \mathrm{cm}^{2}$.

- Small diameter. 
- Low attenuation, low cross-talk, negligible pick-up.

- Impedance uniform to better than $\pm 10 \%$.

- Propagation speed constant so as to induce less than 2 ns time dispersions for $6 \mathrm{~m}$ cables.

- Affordable cost.

After several tests, the choice was made to use $\mathrm{Cu}$-Ag coaxial cables with rolled polyimide as dielectric. For both $25 \Omega$ and $50 \Omega$ cables, the external diameter is $1.2 \mathrm{~mm}$ with a braided outer conductor of $63 \mu \mathrm{m}$ wires. The central conductor is a single wire $200 \mu \mathrm{m}$ in diameter for $50 \Omega$ cables, and a bundle of 7 wires, $127 \mu \mathrm{m}$ diameter each for the $25 \Omega$ cables.

\subsubsection{Connectors}

The end of A harnesses connected to mother boards is a low profile connector, $5 \mathrm{~mm}$ thick, this choice minimizing the dead liquid argon thickness in front of the module. At the other end, at the patch panel, the A and B harnesses are connected through a male-female pair of 100-pin micro-D connectors ${ }^{8}$. While 64 pins are used for signal transmission, ground connections are grouped by 2 on one pin. All grounds are anyhow common at the mother board and at the feedthrough, (see subsection 6.2). Micro-D connectors were chosen for their small size and proven reliability. The last connector, between harness B and the feedthrough is an ATI custom developed connector. Two rows of 32 pins carry the signals, while the ground is channelled on each side of the connector by a spring blade, to the pin carrier of the corresponding feedthrough slot. All contact surfaces and pins are gold plated.

\subsubsection{Quality control}

An automated test bench [14] at the cable production plant ${ }^{9}$ monitored the quality of all cables. Cable coils and/or harnesses not fulfilling the criteria were rejected. Fig. 18 shows the impedance of each cable coil and Fig. 19 shows the distributions obtained for the propagation time along $4 \mathrm{~m}$ long cables. Radiation resistance was assessed by measuring the electrical properties of a given harness before and after irradiation at the Dubna IBR2 reactor. Absence of liquid argon pollution under irradiation was verified using a dedicated setup [18] exposed to a neutron beam at CERI (Orléans, France).

\footnotetext{
8 Micro-D twistpin connectors from ITT/Cannon.

9 Axon' cable SA.
} 


\subsubsection{High voltage cables}

In order to avoid possible HV discharges or Corona effects in coaxial cables, single conductor, PEEK insulated cables were selected for HV connections (see subsection 6.3). HV cables were grouped in harnesses of 8. Each HV cable was soldered to dedicated HV boards at the module level. At the patch panel level, an 8-point custom developed ATI connector ${ }^{10}$ was chosen.

\subsection{Cabling procedure and electrical tests}

The inner radius face of each module was cabled first [15]. To start the summing boards were plugged into the electrode connectors. There are 64 summing boards for the region $0<\eta<0.8$, 96 for the region $0.8<\eta<1.4$ and 8 for the region $1.4<\eta<1.475$. This gives a summation of 16 electrodes in $\phi$ leading to a $\Delta \phi=0.1$ granularity. An electric continuity test ensured that no signal channel was grounded. Thin $(0.3 \mathrm{~mm}) \mathrm{G} 10$ boards (one for each mother board) completely covering the summing boards were installed. The aim of this board is to protect the cables from the contact of the numerous solderings on the summing boards. They were bored with holes for signal and ground connections between the summing and the mother boards. The mother boards were installed by plugging pin carriers going through the mother to the summing boards via the G10 boards. The cable harnesses for signal readout and calibration were installed, the low profile connectors plugged into the mother boards on one end, the other end being mounted on a metallic patch panel located in the large $|z|$ region on the module. The cables were fixed to the thin G10 boards using polyethylene cable ties.

As the calibration pattern shows regularity and periodicity, a connector permutation might not be seen by the electrical tests described below. To eliminate this problem which has consequences on the trigger summation for physics signals, a continuity test was performed, checking that any low profile connector was at its expected position. After the tests were performed (described below), thick ( $2 \mathrm{~mm}$ ) G10 covers were screwed on the internal rings to maintain and protect the front cables.

The outer radius was cabled after putting the module into a manipulation frame and rotating it around the $\phi$ axis. 56 summing boards were installed, summing 4 electrodes in $\phi$ which leads to a $\Delta \phi=0.025$ granularity. This was followed by a shorts-to-ground test. 14 high voltage buses were installed, grouping 32 electrodes in $\phi$ (corresponding to a $\Delta \eta \times \Delta \phi=0.2 \times 0.2$ zone) with 2 lines supplying independently the 2 sides of each electrode. Then 14 thin G10 boards were positioned followed by as many mother boards connected

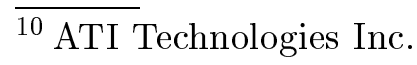


to the summing boards by pin carriers with TVS to protect the calibration resistors against sparks. Six metallic cable trays were placed into the holes in the external rings and fixed in one point using a G10 piece to ensure electrical insulation. In addition they were isolated from the 6 external rings using kapton foil pieces. Signal and calibration cables were placed in the cable trays between the mother boards (Fig. 20) and the patch panels [15]. High voltage cables were installed similarly and were soldered on each high voltage bus.

The zero potential of the calorimeter (absorbers) and the ground of the rings and of the cryostat are connected at the feedthrough level [16]. The cryostat in ATLAS is electrically insulated from its support and from the cryogenic lines.

At this stage three electrical tests were performed [17]:

- A first test (called TPA) consisted in pulsing successively each calibration line with a step pulse (rise time $1 \mathrm{~ns}$, amplitude $40 \mathrm{~V}$ ) and reading all signal lines expecting a signal according to the calibration scheme. Exponential type shaped signals corresponding to the charge of the detector capacitors through the calibration injection resistors were recorded. The analysis of the maximum of the signal shaped with an $\mathrm{CR}-\mathrm{RC}^{2}$ function with $13 \mathrm{~ns}$ shaping time [6] tested the integrity of the whole circuitry, e.g. the calibration resistor system or the connection of the detector capacitor to the mother board. In practice the step signal was generated by mercury relays followed by a $(2 \times$ 64) multiplexer to pulse all calibration lines of half a module. The signal lines were also multiplexed $(25 \times 64)$ and read out by a digital oscilloscope with an automatic procedure. Fig. 21 shows the results of this test for one module. Several types of problems could be detected: no signal showing a break in the readout path, a too high signal showing a reduced detector capacitance due to the detection cell being disconnected from the readout system. Finally a too low signal corresponded usually to a slow rising signal, likely associated with a channel where the braid of the cable was not connected to the detector ground. All these problems were repaired during the cabling steps.

- The second test (called TBF) consisted in injecting a sinusoidal signal into the high voltage line and reading out the signal in the middle layer which has a large detector capacitance. This test checks the connection of the high voltage from the high voltage connector to the electrode high voltage layer. A frequency between 1 and $20 \mathrm{~Hz}$ was shown to be sensitive to one missing electrode out of 4 in one middle cell[17]. Fig. 22 shows the results of this test. The two high voltage lines show different amplitudes due to different values of the decoupling capacitances for the two electrode high voltage sides [12], useful for finding inverted cabling. For sectors cut in 2 parts in $\phi$ the effect on the TBF test is very clear. The case of one isolated electrode is also clearly detectable.

- The third test consisted in applying high voltage to the module. As the 
calibration resistances are sensitive to sparks, this test was performed for only 5 minutes at $1 \mathrm{kV}$ when the readout lines were open at the end and $1.4 \mathrm{kV}$ when they were connected to their characteristic impedance (in order to absorb the spark energy). During the ramping up of the high voltage $(2 \mathrm{~V} / \mathrm{s})$ the currents were $\approx 10 \mu \mathrm{A}$ and the leakage currents $\approx 100 \mathrm{nA}$ afterwards. A high voltage line might show a trip or a short. If it happened at warm, the faulty electrode sector $(\Delta \eta=0.2)$ could be isolated and put on two separate high voltage lines (for the two electrode faces). If a problem appeared during the cold test and disappeared at warm, a high voltage of $2 \mathrm{kV}$ was applied on this zone (shorting the calibration resistors to protect them) to attempt to burn the cause of the problem. To reduce the size of the potentially problematic HV zone, the latter was divided in two independant high voltage zones in $\phi$, which required two additional spare lines for the two electrode sides.

\subsection{Module cold test.}

After having passed the electrical tests performed during the cabling phase, the module performance was verified under cold conditions in liquid argon. Four modules, out of 32, had gone through beam tests in a liquid argon filled cryostat at CERN. The results are reported in section 9. All other modules have been tested in liquid argon at the three assembly sites. The cold tests were performed on dedicated test stations composed of a vertical cryostat with feedthroughs to connect all HV, signal and calibration lines of the module. Unlike the other sites, the test station at CERN used the test-beam cryostat without a beam, with the module in a horizontal position. The tests included:

- High voltage at $2 \mathrm{kV}$ for at least 3 days.

- Electrical continuity verification with a sinusoid wave sent through the HV lines with signal readout (TBF test).

- Calibration circuit verification with a square signal sent through the calibration lines with signal readout (TPA test).

The liquid argon test started with the cool-down at a rate of $2 \mathrm{~K} /$ hour performed by circulating liquid nitrogen in a cooling circuit. This limited the temperature gradient to $40 \mathrm{~K}$ over the whole module. During the tests in liquid argon, all HV currents and test waveforms were recorded. The purity of the argon bath was measured during the test with an ATLAS-type purity monitor (see subsection 5.1), to check against an accidental pollution of the module. The warm-up operation was performed with heaters at a rate of $2 \mathrm{~K} / \mathrm{hour}$ from $90 \mathrm{~K}$ to $300 \mathrm{~K}$. After the test, repairs were done, as explained in the previous section, before shipping the module to CERN, where it was stored in containers permanently flushed with dry nitrogen. 


\section{$5 \quad$ Monitoring instruments}

The cold monitoring components on the barrel calorimeter are of several types: purity monitors, temperature probes, strain gauges and position gauges.

\subsection{Monitoring of the purity of the liquid argon}

Although the sensitivity to electronegative impurities in the argon is reduced by the fast shaping ${ }^{11}$, they can still reduce the amplitude of the collected charge and degrade the performance of the calorimeter. In order to control the argon purity, 10 purity monitors have been installed on the outer radius of the calorimeter between the support rings. They are located in the median azimuthal plane and at the top and the bottom of the half barrels.

The impurity measurement is based on the energy deposition by radioactive sources in the liquid argon. The ionisation charge is collected by an electric field and measured by a cold pre-amplifier. Each device consists of two radioactive sources: an ${ }^{241} \mathrm{Am}$ source emits $5.5 \mathrm{MeV} \alpha$-particles and a ${ }^{207} \mathrm{Bi}$ source $1 \mathrm{MeV}$ conversion electrons. The setup of the monitor is shown in Fig. 23. It consists of signal gaps for the two sources, which are made out of polyimide, high voltage filters and distribution circuits and a single charge sensitive pre-amplifier. Both signals (in the range $2-4 \mathrm{fC}$ ) are read out by this pre-amplifier at the same time. The distinction of the cell is done by the polarity of the signal.

In order to extract the absolute oxygen content in the liquid argon, the ratio of the measured charges $Q_{B i} / Q_{A m}$ is used. This is possible because the dependence of the charge on the impurity level is different for the two sources. An empirical calibration of the signal ratio of the two sources has been performed and the result is shown in Fig. 24. The obtained parametrization as a function of the temperature and the field strength has a total error of $15 \%$ on the absolute level of the oxygen content. Relative changes can be measured with a precision of better than $10 \mathrm{ppb} \mathrm{O}_{2}$-equivalent contamination. More details about the methods used and the performance of the devices can be found in [19].

\footnotetext{
${ }^{11} \mathrm{~A}$ variation of $\pm 0.1 \mathrm{ppm}$ around $0.5 \mathrm{ppm} 0_{2}$-equivalent purity and a shaping time of $20 \mathrm{~ns}$ translates in a $\pm 0.05 \%$ signal variation.
} 


\subsection{Other monitors}

Temperature probes (PT100 platinum resistors) are located in the liquid argon gap, glued on the G10 bar and the absorber in order to measure the liquid argon temperature where the ionisation takes place. There are 6 such calibrated [20] $(\Delta T=10 \mathrm{mK})$ probes per module located in its median azimuthal plane, 3 at $R=1500 \mathrm{~mm}$ and 3 at $R=2000 \mathrm{~mm}$ regularly distributed in $z$, for a total of 192 probes for the whole barrel. In addition 40 probes are located on the cable trays in order to measure the temperature outside of the calorimeter and 32 probes are located on the two rings at largest $|z|$ to measure their temperatures during the cooling process. Finally, 8 probes have been installed on the 4 rails supporting the two barrels to monitor the cooling process.

Strain gauges (16 in total) are glued on the external rings 2 and 5 at azimuthal positions $|\Delta \phi|=\pi / 8$ apart from the horizontal plane where the maximum effect is expected. These gauges are monitored when a half barrel is rotated from the vertical to the horizontal position.

In each half barrel, 4 LVDT type position monitors are fixed on the $\eta=0$ external ring. They allow to measure the distance of this external ring to the cryostat middle flange. They have been beforehand adjusted in such a way that one is able to position the beginning of the absorber G10 bars at $|z|=2 \mathrm{~mm}$. They are used while the half barrels are being inserted into the cryostat and also during the cooling process and during the transportation towards the experimental zone.

\section{Cryostat and feedthroughs}

\subsection{Barrel cryostat}

The barrel cryostat ${ }^{12}$ (Fig. 25) [21] can be described as a ring dewar made out of 4 nested aluminium cylinders named respectively outer warm, outer cold, inner cold and inner warm cylinder ${ }^{13}$. Aluminium ${ }^{14}$ has been chosen in order to reduce the total number of radiation lengths.

\footnotetext{
${ }^{12}$ Overall length $\sim 6.80 \mathrm{~m}$, the diameter of the inner and outer warm cylinders are respectively $\sim 2.30 \mathrm{~m}$ and $\sim 4.50 \mathrm{~m}$.

13 The inner warm cylinder supports in addition the central solenoid on its exterior, i.e. the central solenoid is housed in the insulating vacuum of the cryostat. The inner warm cylinder will also support the ATLAS inner detector in its inner bore.

${ }^{14}$ Aluminium 5083.
} 
The outer and inner cold cylinder are closed at both ends with a bulkhead creating the so-called cold vessel. In the same manner the outer warm cylinder and inner warm cylinder are closed with two bulkheads creating the surrounding warm vessel. All bulkheads are circular shaped plates with passage holes to allow the insertion of the inner detector into the inner warm cylinder bore. The cold bulkheads are bolted and welded ${ }^{15}$ to the cold cylinders, whereas the warm ones are bolted and vacuum sealed with O-rings. All four bulkheads and the inner cold vessel have tapered walls to minimize the material between the interaction region and the electromagnetic calorimeters.

The cold vessel rests on four feet made out of G10 providing electrical and thermal insulation. They will shrink during cool down causing the cold vessel to sag by about $4 \mathrm{~mm}$ eventually. Three keys, two acting laterally and one acting longitudinally maintain its position. Clearances between keys and keyways were necessary to allow the assembly of the cryostat. These horizontal clearances will shrink during cryostat cool down but there will be remaining plays of about $\pm 1.5 \mathrm{~mm}$ - they must be regarded as position uncertainties. Therefore, position sensors have been installed between warm and cold vessel to measure possible movements of the cold vessel, allowing to eliminate these position uncertainties for the alignment in ATLAS. In order to reduce radiative heating of the cold mass and thus the required cooling power, a multi-layer insulation system is installed on the cold vessel walls.

In ATLAS, the barrel cryostat will be collinearly centred in the inner bore of the tile barrel calorimeter. Because the barrel cryostat must not touch the tile barrel calorimeter for mechanical and electrical reasons, the design includes a surrounding gap of about $30 \mathrm{~mm}$. With the aim to achieve an optimal force transmission for a 4-point support, the outer warm cylinder is equipped at the lower half of its two ends with four support interfaces at $\pm 45^{\circ}$, providing sliding surfaces at $\mp 45^{\circ}$. The entire barrel cryostat load ${ }^{16}$ is transferred via these interfaces and four support plates (Fig. 26) directly to the saddles that support the tile barrel, so that no load is applied to the tile barrel itself.

The cryostat was subjected to numerous tests. Several leak checks and pressure tests have been performed, among them a 3.5 bar (25\% above the maximum operating value) test for the cold vessel. The measured bulkhead and cylinder deflections were all found to be in agreement with finite element calculations. The cryostat was cooled down to $80 \mathrm{~K}$ and filled half-way with $\ell \mathrm{N}_{2}$. No cold spots were found, demonstrating the effectiveness of the insulation in particular at the level of centring keys and feet. The heat conductance was estimated to be no more than $1 \mathrm{~W} / \mathrm{m}^{2}$.

\footnotetext{
${ }^{15}$ For cold testing however, the cold vessel was sealed with provisional Omega seals.

16 The overall weight is about 206t - cryostat $\sim 25 \mathrm{t}, 2$ half barrel calorimeters $\sim 2 \times 57 \mathrm{t}$, liquid argon $\sim 56 \mathrm{t}$, central solenoid $\sim 6 \mathrm{t}$ and inner detector $\sim 5 \mathrm{t}$.
} 


\subsection{Signal feedthroughs}

64 signal feedthroughs bring 122880 signal, monitoring, calibration and spare lines through an insulating vacuum, out of the liquid argon environment, into the warm one. To have connection lines as short as possible, there are 32 signal feedthroughs for each half of the barrel calorimeter, distributed radially around each end of the barrel cryostat. They have been designed to minimize heat conductance, to accommodate $\mathrm{a} \approx 1.5 \mathrm{~cm}$ displacement during cooldown and to minimize the cable lengths as well as the cross-talk level while ensuring an excellent ground return.

A feedthrough, Fig. 27, consists primarily of a warm flange and a cold flange, with a flexible bellows welded between them and pumped down to about $10^{-3}$ mbar vacuum. Each flange houses 4 pin carriers with a total of 30 insulating glass rows, with 64 gold-plated pins per row, providing a total of 1920 signal connections per feedthrough. Heaters $(15 \mathrm{~W})^{17}$ located on the warm flange prevent condensation and frosting. Both flanges are interconnected with flat, polyimide strip-line flexible $33 \Omega$ cables. Special monitoring and control lines for the feedthroughs, which do not enter the cryostat volume, are passed through a filter and follow strict grounding protocols, to prevent noise from being introduced to the signal readout. The feedthrough design is robust in that a few feedthroughs with substantial leaks in their bellows volume can be accommodated without compromising the operation of the calorimeter.

Each signal feedthrough was cold-cycled and thoroughly leak-tested. A full set of electrical tests was performed during these test cycles as well as during the entire assembly and installation process: the electrical continuity and characteristics of each line were tested with commercial continuity and timedomain-reflectometry testing equipment. More details on the construction and the tests of signal feedthroughs can be found in [22].

\subsection{High voltage feedthroughs}

A single HV feedthrough port [23] is mounted on each end of the barrel cryostat, close to its highest point. Each port holds about $840 \mathrm{HV}$ wires, divided over four wire feedthroughs. Of these, 714 wires serve the standard HV lines ${ }^{18}$, the remainder is spares and used to supply single electrodes or split sectors (see Section 4.3). Each port consists of a single warm bulkhead erected on

\footnotetext{
${ }^{17} \mathrm{Up}$ to $200 \mathrm{~W}$ can be provided in case of a leaking feedthrough. 18 The half barrel segmentation is $32 \times 7 \times 2=448$ channels $(\phi \times \eta \times 2$ electrode sides), the corresponding preshower segmentation is $32 \times 4 \times 2=256$ channels and the purity monitors need $10 \mathrm{HV}$ lines.
} 
the warm vessel from which the wire bundle leads to the calorimeter via a tube connected to the cold vessel by stainless steel bellows, which provide the bulk of the heat resistance. The bellows accommodate the relative $\approx 1.5 \mathrm{~cm}$ displacement of the cryostat vessels during cooldown. The liquid rises up to the bellows followed by a $70 \mathrm{~cm}$ high column of argon gas at 1.25 bar. Under these conditions, the heat leak is calculated to be $1 \mathrm{~W}$ per port. Warm O-rings and RF gaskets between the ports and the warm vessel ensure gas-tightness and close the Faraday cage at the level of the HV filters mounted on top of the HV feedthrough ports.

The HV wire consists of a solid $0.41 \mathrm{~mm}$ diameter constantan conductor ${ }^{19}$ with a $0.30 \mathrm{~mm}$ thick PEEK (PolyEther-Ether-Ketone) cladding ${ }^{20}$, chosen for its radiation hardness. All wires are stripped inside the wire feedthroughs and potted, insuring a full wire continuity between the warm connectors ${ }^{21}$ and the cold connectors on the calorimeter patch-panels. As there is no ground return on the $\mathrm{HV}$ wires, great care has been taken in certifying the wire for absence of corona.

The wire feedthroughs have been extensively tested, in particular with argon at standard temperature and pressure on the cold side and $\geq 60 \% \mathrm{RH}$ air on the warm side of the wire feedthrough. $3 \mathrm{kV}$ was applied at each individual $\mathrm{HV}$ wire in turn, keeping all other wires at ground. Wires showing more than $2 \mathrm{nA}$ of leakage current were either repaired or rejected (less than 5 such wires per feedthrough found). After assembly, the feedthrough bulkheads were fully He leak checked. After installation, the wiring was tested again at $2.5 \mathrm{kV}$, and checked for correct mapping and electrical continuity. More details on the HV feedthroughs can be found in [23].

\section{$7 \quad$ Half barrel assembly}

\subsection{From the modules to the final half barrel}

16 modules are required to assemble a cylinder, also named 'half barrel'. The assembly was done in vertical position inside a clean room ${ }^{22}$ that provided sufficient space for module+backbone manipulation, for cylinder assembly on a special table and for storing a half barrel on a support stand after rotating it into horizontal position.

\footnotetext{
19 The resistor is $3 \Omega / \mathrm{m}$.

${ }^{20}$ Habia Cable, Söderfors, Sweden.

${ }^{21}$ REDEL Kft S-series 51-channel 3kV connectors, model SLA.H51.LLZG, Lemo SA, Switzerland.

${ }^{22}$ Class ISO 8, temperature $(20 \pm 1)^{\circ} \mathrm{C}$ and humidity $(<50 \%)$ controlled.
} 
To ensure the closing of the cylinder, a geometrical survey in $R, r, z, \phi$ (large and small cylinder radii, cylinder axis and azimuthal angle) was put in place. The first step consisted in the materialization of the cylinder axis by creating a set of reference points in the clean room. Then reference points were also placed on the external and internal ring-pieces of each module to be able to position each module to a precision better than $0.3 \mathrm{~mm}$ in space.

Before rotating the module+backbone set to the vertical position, spurious groundings were searched for and eliminated, and an HV test at $1000 \mathrm{~V}$ was performed. The first module+backbone set was put in vertical position and secured on the assembly table. This table is made of 2 half stands, one was fixed on the floor and the other was mobile to allow the opening or closing of the cylinder. The next module+backbone set was prepared and just before being put vertical, the 65th absorber of the preceding module was removed, opening the gap common to the two modules (Fig. 29). Elastic dowel pins were inserted in the G10 bars and stainless steel dowel pins were inserted in the external ring-pieces in order to block the radial translation of one module with respect to the other. The positions in $R$ and $z$ were given by the indexation of dowel pins common to two consecutive backbones. The backbones have been indexed during their machining in the factory. The two modules were joined inside the clean room using a crane and the hinges on the rear face of the backbones. When the hinges were in contact, they were used as a rotation axis, the closing plane going from the backbone rear diameter towards the internal radius of the module, then fitting in regularly the accordion zigzag. The screws linking the backbones were tightened and the $\mathrm{Cu}-\mathrm{Be}$ bolts linking the external ring-pieces were put in place. The linking of the internal ringpieces was left free and the clearance at small and large radius, $r$ and $R$, was measured and registered. An additional HV test at $1000 \mathrm{~V}$ was performed to check that the gap between the two modules which had been opened still held the high voltage.

The difficulty was to close the cylinder with a minimal clearance at the internal radius $r$. This clearance was due not only to the mounting clearance (varying with $z$ ) but also to the variation of the absorber span due to the cold test in the test cryostats and to the bulging in vertical position. These parameters had been carefully measured after the cold test. Furthermore, once a new module+backbone set was added to the cylinder, the clearance with respect to the preceding module and the position of the new module was measured. Taking into account all these parameters the position of the next module was defined. The first 15 modules were assembled as defined above. For the last module (because of accordion shape), one needed to reopen the cylinder ( 8 modules on the fixed half stand and 7 on the mobile half stand) in order to be able to position the last module. Once done, the mobile half stand was moved to close the cylinder again. 
When fully assembled, the screws between backbones and those between external ring-pieces were tightened to the desired torque. A general map of the clearances at small radius was measured. Jigs installed on the internal ringpieces ensured that the clearances were equally shared between modules. These final clearances were measured and the titanium links between internal ringpieces were machined accordingly and then fixed on the ring-pieces. The last operation done with the cylinder axis vertical was the installation of presampler rails. The mounting rails for the presampler were screwed on vertically to the internal rings of the calorimeter with a clearance of $0.1 \mathrm{~mm}$. Since the rails are made of a material which has a lower shrinkage coefficient than the absorber bars, all mounting holes except the one at $\eta \simeq 0$ are oblong to let the rails shrink differently than the calorimeter.

Before rotating the cylinder, it was hermetically sealed for dust protection. The roof of the clean room was opened to use the hall cranes. To rotate the cylinder, a jig fixed to the lateral backbones was used (Fig. 30). The cylinder with its axis horizontal was put on a dedicated stand.

To insert the presampler, an adjustable platform was built. The position and the height of the platform were adjusted in front of the half barrel. The aluminium profile containing a presampler sector was then installed onto an insertion tool placed on the platform (Fig. 31). The position, alignment and tilt of the insertion tool and the profile, i.e. the sector, was adjusted using a laser pointing system. The presampler sector was pulled out of the aluminium storage profile with a cable attached to a winch and thus pushed onto the rails at the internal face of the calorimeter like a drawer. Finally, the presampler sector was attached to the calorimeter at $\eta=0$. The gap between neighbouring sectors is nominally $0.4 \mathrm{~mm}$ at room temperature and it turned out to be necessary to adjust the shell tightly around each presampler to maintain sufficient clearance.

After the insertion of each presampler sector, its electrical connections were systematically tested by injecting low frequency sinusoidal signals and test pulse signals in a similar way as for the validation tests at the production sites. An insulation test at $1.4 \mathrm{kV}$ was also performed for at least $1 / 2$ hour. In a few instances when problems were found (three HV short circuits and one broken solder joint), the sector was removed and repaired or replaced by a spare one.

The last operations consisted in the installation of the heat exchangers through the $\eta>0$ external rings, of the $\eta=0$ external ring equipped with position monitors and of the sliding rails needed for the insertion of the calorimeter inside the barrel cryostat. The $\eta=0$ ring (very close to the plane $z=0$ ) was fixed to the sliding rails, whereas the other rings, thanks to oblong holes, are allowed to slide on the sliding rails during the cool down. As the sliding rails 
were made of the same aluminium alloy as the cryostat itself, one can fix them at the end of the cryostat and have a negligible displacement of the $\eta=0$ ring with respect to plane $z=0$ during the cool down. The calorimeter was decoupled from the 10 bottom backbones before being transported.

\section{Detector insertion into the cryostat - warm and cold tests}

\subsection{Half barrel insertion, connection and test}

In order to insert a half barrel into the cryostat an insertion stand was set up in front of the open cryostat - a massive support structure with rails of similar shape as the support rails inside the cryostat. Stand and rails were aligned with respect to the cryostat rails. The half barrel was lifted out of the assembly clean room with an overhead crane (one wall and the ceiling had to be dismounted therefore) and placed on the insertion stand. The remaining 6 backbones were then removed. A temporary clean room was erected enclosing the insertion stand, the half barrel and the cryostat opening.

Before pushing the half barrel inside the cryostat several tests have been carried out. At first a high voltage test was performed applying up to $1000 \mathrm{~V}$ to the EM calorimeter and Presampler. High voltage problems which had occurred during the half barrel assembly, rotation and transportation were then treated with the techniques described in section 4.3 for one single module.

Finally the electrical insulation of the calorimeter ground (absorbers) relative to the external rings was verified. The half barrel and its associated aluminium sliding rails were inserted inside the cryostat by sliding them onto the cryostat rails. The half barrel was positioned at $2 \mathrm{~mm}$ from the $z=0$ plane thanks to the position monitors and the sliding rails were fixed on the cryostat rails at $z=3200 \mathrm{~mm}$.

Different geometrical measurements were then performed. Using the reference points placed on the external and internal absorber G10 bars during the half barrel assembly, the circularity of the half barrel at the inner and outer radius and at $z=0 \mathrm{~mm}$ and at $z=3200 \mathrm{~mm}$ was measured. The deformation due to the weight of the calorimeter was clearly seen (Fig. 32) and is in a fair agreement with a finite element calculation as shown in Fig. 15 for the outer radius for both half barrels. The centers of the fitted inner and outer circles coincide to better than $0.5 \mathrm{~mm}$. Finally the sagging of the absorbers was measured as a function of the azimuthal angle, with a maximal value of $2.5 \mathrm{~mm}$ near the horizontal plane. 
Due to difficulties in the positioning of the half barrels inside the cryostat, both half barrels will be at cold $4 \mathrm{~mm}$ too low and $2 \mathrm{~mm}$ laterally displaced with respect to the cryostat's central axis [24]. Part of the vertical misalignment will be compensated by positioning the cryostat inside ATLAS about $2 \mathrm{~mm}$ higher than originally planned. The remaining misalignments will be corrected by software.

The electrical insulation was checked again before connecting the feedthrough harnesses. These cables were first checked for possible permutations not detectable by the calibration tests as described in section 4.3. In total for each half barrel, 960 signal harnesses and 96 high voltage harnesses were connected.

The standard sequence of tests (TPA, TBF, high voltage at $1400 \mathrm{~V}$ ) was performed from the base planes of the front-end crates, the front-end electronics being installed later. TPA test results for the entire calorimeter, including the presampler, are shown in Fig. 33. Acceptation criteria for missing channels have been defined beforehand and were compared with the test results in Table 3. The number of dead signal channels for calibration over the entire calorimeter is below the rejection thresholds except for the barrel end $(0.15 \%$ instead of $0.05 \%$ ). There were no attempts to repair these faulty channels, as it was considered too risky for the detector.

The TBF tests were performed. Pulsing a HV line with a low frequency signal and reading out the associated signal cells allows a full debugging of the geometrical mapping of the HV lines, especially the spare ones.

No additional high voltage problem at $1400 \mathrm{~V}$ appeared after insertion of the calorimeter in the cryostat. In total, 21 electrode sectors $(\Delta \eta=0.2)$, out of a total of $14336(0.15 \%)$, having shown a problem in one side at warm are powered separately. Furthermore 23 electrode sectors, out of a total of 448, which had a problem only at cold have been subdivided in $2(2.6 \%$ of half sectors). The effect of not supplying high voltage to one side of the electrode was studied in the test beam. A slight deviation to the expected factor 2 in the signal height observed at the transition region with a good sector was understood as a geometrical effect and is well reproduced by simulation and is therefore easy to correct for. For the presampler, out of almost 44000 anodes, 6 were completely disconnected due to HV short circuits, 5 anodes had one side disconnected for similar reasons. In addition $18 \mathrm{HV}$ resistors (out of almost $175000)$ showed a bad contact after installation in the cryostat, i.e. in total less than $0.03 \%$ of the HV resistors are removed or faulty.

The LC value, product of the inductance by the capacitance of a readout cell, needs to be known to better than $1 \%$ to achieve precise signal reconstruction [28]. Moreover, a control of the final geometry was performed comparing the capacitance values with expectations. The measurements proceeded as 
Table 3

Table showing the number of channels in each calorimeter layer, the predefined acceptable maximum rate and number of faults, the faults found after insertion at warm and afterwards at cold.

\begin{tabular}{|l|l|l|l|l|l|}
\hline Layer & Nb of channels & $\begin{array}{l}\text { Max. rate } \\
\text { accepted }\end{array}$ & $\begin{array}{l}\text { Max. nb. } \\
\text { accepted }\end{array}$ & $\begin{array}{l}\text { Nb. found } \\
\text { at warm }\end{array}$ & $\begin{array}{l}\text { Nb. found } \\
\text { at cold }\end{array}$ \\
\hline presampler & 7808 & $0.05 \%$ & 4 & 0 & 0 \\
strips & 57216 & $2 /$ mod. & 64 & 9 & 11 \\
middle & 28672 & $0.05 \%$ & 14 & 2 & 5 \\
back & 13824 & $0.05 \%$ & 6 & 0 & 4 \\
barrel-end & 2048 & $0.05 \%$ & 1 & 3 & 3 \\
calibration & 8192 & $0.05 \%$ & 4 & 1 & 1 \\
\hline \hline
\end{tabular}

follows: a sinusoidal signal produced by a network analyser, with frequencies ranging from $10 \mathrm{kHz}$ to $150 \mathrm{MHz}$ by steps of $100 \mathrm{kHz}$, was injected into the calibration input of the base-plane connectors. The output signal had a resonance frequency which gave the desired information $\left(\nu_{0}=1 /(2 \pi \sqrt{L C})\right)$. All readout cells of middle and back layers of the central calorimeter were measured at room temperature. The obtained output signal is shown in Fig. 34, for a typical middle cell. The resonance frequency $\nu_{0}$ is plotted as a function of the middle cell position in $\phi$ in Fig. 35, for cells belonging to the four middle regions $\eta<0.4,0.4<\eta<0.8,0.8<\eta<1.2$ and $1.2<\eta<1.4$. Inside each such region, the observed small variation in $\phi$ agrees with the inductance variation from channel-to-channel due to the summing board. The absolute resonance frequency decreases from the first to the last region, as expected from the increasing cell capacitance with pseudorapidity.

Similar operations (cable connections, TPA, TBF, HV and LC tests) were repeated for the second half barrel. All this work was performed in a closed and clean environment, without any specified cleanliness classification.

\subsection{Calorimeter cooling operations}

Before cooling the barrel calorimeter, it was necessary to remove all the contaminants present in the cryostat or in the calorimeter. This was achieved by pumping and then filling the cryostat with clean and dry argon gas. This operation was repeated until the remaining traces of air and water in the argon contained in the cryostat correspond to a purity better than $100 \mathrm{ppb}$ of $\mathrm{O}_{2}$-equivalent. 
The cryostat and the calorimeter are made of materials having different thermal coefficients. Therefore small temperature differences may induce unacceptable constraints or displacements. For example the two half barrels lie on aluminium cryostat rails and are fixed at the end of these rails. Under small temperature differences between the half barrels and the cryostat, the two half barrels may come closer to each other and can be damaged.

In total 7 temperature and 8 displacement criteria were defined to form a common interlock stopping the cooling process. Thermal models [26] were made, by decomposing the calorimeter into several subsets (cryostat, external rings, absorbers, internal rings) to estimate a reasonable exchange power $(4 \mathrm{~kW})$ in a free convection mode. A temperature difference $(\delta \mathrm{T}=40 \mathrm{~K})$ between the input and the output of the heat exchangers satisfies the above temperature criteria and allows to extract the $8 \mathrm{GJ}$ of the cryostat and of the calorimeter at a speed of $0.25 \mathrm{~K} /$ hour. The calorimeter cooling indeed lasted 800 hours.

From warm temperature down to $120 \mathrm{~K}$ the cooling procedure was achieved by a forced circulation of cold gaseous nitrogen in the heat exchangers of the half barrels ( 4 in total). By adjusting the temperature of the incoming cold nitrogen and its flux, one was able to satisfy the $\delta \mathrm{T}=40 \mathrm{~K}$ condition. The cryostat was filled with gaseous argon maintained at a pressure of $1300 \mathrm{mb}$. At about $120 \mathrm{~K}$, the exchange power became ineffective, therefore one circulated pressurized liquid nitrogen in the heat exchangers instead of gaseous nitrogen.

At the end of the cooling, around $90 \mathrm{~K}$, a small amount of argon (about 100 l) was liquefied in order to homogenize the temperature inside the cryostat. This was done to ease the transfer to the cryostat of the necessary 400001 of liquid

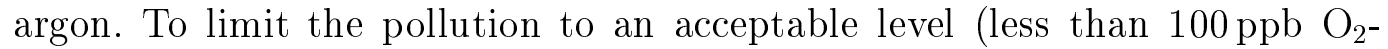
equivalent) the liquid transfer was done through an oxygen filter.

The temperature stabilisation inside the cryostat was achieved by adjusting the pressure and the liquid percentage of the nitrogen circulating in the 4 calorimeter heat exchangers and the 2 feedthrough heat exchangers. The temperature differences between the input and output of the heat exchangers were small, therefore meaning that all the $\delta$ Ts in the calorimeter were also small. The total heat leak was estimated to be less than $2 \mathrm{~kW}$ in agreement with expectations. The non-uniformity of the argon bath in this free convection mode was less than $500 \mathrm{mK}$ between the top and the bottom of the calorimeter and less than $50 \mathrm{mK}$ between the 2 half barrels and between the left and the right part of the calorimeter.

After emptying the cryostat of the liquid argon, the calorimeter underwent the warming up process. The warming power $(4 \mathrm{~kW})$ was supplied by resistors lying on the bottom of the cryostat outer cold cylinder. The warming operation respected the same temperature and displacement criteria defined for the 
cooling operation. Fig. 36 shows the temperature variation from the cooling down to the warming up of the calorimeter and the temperature difference between the warmest and the coldest temperature probes of the calorimeter, including its rings and supports.

\subsection{Calorimeter cold tests}

The cryostat stayed full of liquid argon for 2,5 months. During that period a sequence of electrical tests and measurements was performed on the detector to qualify it. As a first step, the nominal high voltage $(2000 \mathrm{~V})$ was applied on the entire calorimeter and presampler. However, a reduced voltage $(1200 \mathrm{~V})$ was applied on sectors cut in two parts (see subsection 4.3). Short circuits were found on seven $\mathrm{HV}$ lines corresponding to $\Delta \eta \times \Delta \phi=0.2 \times 0.2$ sectors, on one line corresponding to a $\Delta \eta \times \Delta \phi=0.2 \times 0.1$ half-sector and on a few isolated electrodes. (No acceptance loss is expected as it concerns only one side of the electrodes). The voltage was applied on all valid channels for an averaged duration of 10 full days with a satisfactory stability in time.

The purity of the liquid argon was measured over the full period with the 10 monitors located in the cryostat. The purity was found to be stable in time with a value around $\approx 100-150 \mathrm{ppb}$ of $\mathrm{O}_{2}$-equivalent.

The TPA test was repeated as before cooling. A total of 23 dead channels (11 in the strip layer, 5 in the middle, 4 in the back, 3 in the barrel end and one open calibration line affecting 8 back channels) were found. This is below the level of 0,5 per mil given as acceptation criteria (see Table 3 ).

Other measurements to electrically qualify the system were undertaken. The calibration and adaptation resistances were measured with a 2 point method from the baseplane. This was performed using a precision ohmmeter, allowing to determine all resistor values to better that $0.1 \%$. The LC measurement was repeated. Finally time-domain-reflectometry (TDR) measurements on all the calibration channels will allow to understand the attenuation and the behaviour of these lines. These were also performed on dead signal channels in order to determine the location of the problem. All these data will be used later to understand and improve the signal reconstruction and the calorimeter response.

A front-end electronics crate, covering a zone $\Delta \eta \times \Delta \phi=1.4 \times 0.2$, or half a module, was installed. The final front-end electronics [25] was tested as well as its associated final low voltage power supplies. The incoherent and coherent noise levels recorded in the three gains were fully compatible with the design values, in the presence of all the monitoring probes and with the high voltage system connected and operational. 
In addition the central solenoid embedded in the cryostat was tested at 1.9 Tesla for 5 hours. Under this condition high voltage was partially raised on the calorimeter with no problem and the purity monitors were demonstrated to work correctly. Finally the effect of the field on the temperature probes was found to be negligible.

\section{Test beam results}

Four series modules, including the associated presampler modules, have been subjected to test-beam, with a setup including a dedicated cryostat and moving table allowing detailed position scans. These tests have been carried out on CERN's SPS H8 beam line, using electron and positron beams with various energies. A comprehensive analysis of module performance, including the data from the four tested modules together, is being finalised [27]. Of utmost importance to the physics performance of the finished calorimeter are the energy resolution and response uniformity. Since these performance parameters depend heavily upon the quality and reproducibility of the construction of the detector, we present here some testbeam results related to the resolution and to the response uniformity. At low energy, the calorimeter response to minimum ionizing particles has allowed us a detailed exploration of the material structure of the detector.

The energy resolution has been studied using electron beams of energies varying from 10 to $245 \mathrm{GeV}$. Electron candidates have been selected by requiring a minimum amount of reconstructed energy in the calorimeter. Pions, muons and off-momentum particles in the beam have been removed by applying various quality cuts on the information from the beam instrumentation. The electron energy is reconstructed by summing the calibrated energies deposited in the various calorimeter compartments. In the middle compartment, the energies of $3 \times 3(\eta \times \phi)$ cells, centered around the cell with the largest energy deposit, are summed together. The energies deposited in clusters in the other compartments, $24 \times 1$ cells in the front compartment, $2 \times 3$ cells in the back compartment and $3 \times 1$ cells in the presampler are summed together. These cluster sizes have been chosen to cover at least the same area as in the middle compartment, and are similar to those foreseen for ATLAS. The fraction of the total electron energy collected within the whole cluster amounts to more than $90 \%$. To correct for the energy loss upstream of the calorimeter (mainly due to the cryostat walls), for longitudinal leakage at the back of the detector, and in order to optimize energy resolution, weights have been applied to the energy deposited in the presampler and back compartment. The experimental 
measurements, after noise subtraction, have been fitted with the expression :

$$
\frac{\sigma_{E}}{E}=\frac{a}{\sqrt{E}} \oplus b
$$

where $a$ is the stochastic term, and $b$ the local constant term reflecting local non-uniformities in the response of the calorimeter. The energy $E$ is expressed in $\mathrm{GeV}$. Fig. 37 shows a typical fit result for one beam position in $\eta$, $\phi$ with $a=9.4 \%$ and $\mathrm{b}=0.1 \%$, the constant term is relative to only one cell. All these results are in agreement with dedicated Monte-Carlo simulations of the test-beam setup and with measurements done with a full size prototype [28].

The response uniformity has been measured using an electron beam of 245 $\mathrm{GeV}$. Non-uniformities in the detector response, coming from mechanical, geometrical, calibration and material effects, translate into a constant term in the energy resolution formula. The ATLAS goal is to achieve a constant term of $0.7 \%$ or smaller over the full calorimeter acceptance. The measured cell non-uniformity is $0.6 \%$ as shown in Fig. 38. This result was obtained by considering 515 middle compartment cells which corresponds to a whole module, excluding cells close to the edges and those close to the transition between electrodes A and B. The performance obtained, both for the energy resolution and for the response uniformity, meet the physics requirements.

Since muons are non interacting particles, their energy deposit is much more localized than the electron energy deposits. Typically, they only cross one middle cell in $\eta$ and 1 or 2 in $\phi$. They can be used to study the fine structure of the calorimeter, without having to deconvolute the effects of the showering process. Fig. 39 left shows the muon energy deposit in the front compartment as a function of $\eta$. The variation with $\eta$ is linked to the depth of this compartment (cf Fig. 7). The signal over noise ratio, evaluated as the ratio of the most probable energy deposit divided by the r.m.s of the pedestal, goes from 7 to 12 for middle cells. Fig. 39 right shows the signal for the $\eta=0.6$ cell.

\section{Conclusions}

The physics to be studied at the LHC imposes very stringent constraints on the electromagnetic calorimetry. Furthermore the harsh environment of the LHC requires the techniques to be pushed to the limit. These considerations led ATLAS to choose a liquid argon / lead sampling calorimeter with an accordion geometry. Four out of 32 series modules have been calibrated with electrons. The results obtained on the uniformity response, the energy and position measurements meet the needed specifications. The construction of the barrel calorimeter has taken eight years from the first designs to the finished detector. All along the construction, from component construction, 
module assembly, cylinder assembly to barrel insertion inside the cryostat, quality assurance measures were taken to minimize detector imperfections. The same care was brought to the construction of the cryostat and associated feedthroughs. At the end of the construction phase, i.e. after the cold test of the whole barrel, the number of defects, such as dead signal channels or faulty HV sectors, is well below the predefined acceptation criteria. The next important step is the installation in the ATLAS pit in its final position.

\section{Acknowledgements}

We are indebted to our technicians for their excellent contribution to the construction and the running of the barrel modules, of the barrel cryostat, of the feedthroughs and of the associated electronics.

We would like to thank J. Ballansat, J-F. Ballansat, J-Ph. Baud, P. Baudin, Y. Beeldens, J. Boniface, H. Bonnefon, P. Brion, G. Broutinel, M. Cailles, V. Clap, L. Journet, P. Letournel, D. Stanchina and J. Tassan from LAPP (Annecy), L. Baisin, L. Bonnefoy, B. Duthion, R. Huber, G. Juban, P. Lançon, J. P. Recour, G. Sannier, W. Seidl, A. Sigrist, W. Wilkens and E. Wood from CERN (Geneva), A. Béteille, R. Blanc, B. Boyer, P. Cavalli, L. Eraud, J-P. Girard, A. Menthe, G. Mondin, S. Muggeo, A. Patti, J-P. Scordilis and M. Yamouni from LPSC (Grenoble), A. Andreani, R. Bertoni, G. Braga, F. Manca, B. Monticelli and F. Sabatini from Milano, L. Bonnemaison, J. Bo-

nenfant, C. Bourgeois, P. Cornebise, O. Dalifard, N. Gregurict, P. Hallin, E. Jules, P. Monneaux, T. Roulet and A. Thiébault from LAL (Orsay), Ph. Beauchet, A. Commerçon, G. Descote†, Ph. Etienne, Ph. Laloux, D. Laporte, Ph. Repain, D. Steyaert and Y. Tréguier from LPNHE (Paris), G. Decock, J.L. Dominique, P. de Girolamo, P. Hardy, A. Marcel, V. Marques, J.P. Mols, G. Prono and F. Senée from DAPNIA (Saclay) and J. Thadome from Wuppertal university.

The construction of the electromagnetic barrel calorimeter would not have been possible without the important financial support of our funding agencies. This support is kindly acknowledged.

\section{References}

[1] ATLAS Collaboration, ATLAS : Detector and Physics Performance ; Technical Design Report, Volume I and II, CERN LHCC-99-014 and CERN LHCC-99015.

ATLAS Collaboration, Calorimeter Performance Technical Design Report, CERN LHCC-96-040. 
[2] RD3 Collaboration, Nucl. Instrum. Methods A 309 (1991) 438.

[3] RD3 Collaboration, Nucl. Instrum. Methods A 321 (1992) 467.

[4] RD3 Collaboration, Nucl. Instrum. Methods A 325 (1993) 118.

[5] RD3 Collaboration, Nucl. Instrum. Methods A 364 (1995) 290.

[6] ATLAS Collaboration, Liquid Argon Calorimeter Technical Design Report, CERN LHCC-96-041.

[7] M.L. Andrieux et al., Nucl. Instrum. Methods A 479 (2002) 316.

[8] B. Lund-Jensen et al., The Printed Circuit Board Electrodes for the ATLAS Liquid Argon Barrel Presampler, paper in preparation.

[9] F. Fleuret et al., Constant Term Evolution with Lead Plate Thickness Fluctuations in EM Module 0, ATLAS note ATL-LARG-2000-002, september 1999 .

[10] B. Mansoulié, Non-uniformity of lead plates and gaps : analytical estimates of the shower averaging effect, ATLAS note ATL-LARG-98-099, may 1998.

[11] J.P.Baud et al, Load tests of the external rings of the barrel EM calorimeter, ATLAS note EDMS ATL-AB-EN-0025, november 2001.

[12] B. Aubert et al., Nucl. Instrum. Methods A 539 (2005) 558.

[13] F. Berny et al., A Protection Scheme for the Calibration resistive Networks, ATLAS note ATL-LARG-2003-005, october 2002.

[14] W. Bonivento et al., Nucl. Instrum. Methods A 451 (2000) 492.

[15] P. Perrodo et al., Cabling procedure for the ATLAS electromagnetic barrel calorimeter modules, ATLAS note ATL-AB-EN-0014, november 2001.

[16] H. Williams, ATLAS policy on grounding and power distribution, ATLAS note ATL-ELEC-98-004, june 1997.

H. Williams, ATLAS policy on grounding and power distribution, ATLAS note ATL-GE-ER-0001, june 1999.

[17] J. Boniface et al., Test bench of the barrel calorimeter modules, ATLAS note ATL-LARG-2001-007, february 2001.

[18] J. Collot et al., Nucl. Instrum. Methods A 350 (1994) 525.

[19] M. Adams et al., Nucl. Instrum. Methods A 545 (2005) 613.

[20] V.Datskov et al., Calibration of temperature probes for the ATLAS liquid argon calorimetry, ATLAS note in preparation

[21] J. Sondericker et al., Design of the ATLAS Barrel Cryostat, ATLAS note EDMS ATL-AB-CD-0002, december 2003.

[22] D. Axen et al., Rev. Sci. Instrum. 76, 063306 (2005). 
[23] M.Rijssenbeek et al., High Voltage Feedthroughs for the ATLAS liquid argon calorimeters ATLAS note in preparation.

[24] ATLAS, LArg barrel assembly in hall 180, summary of the geometrical axis and main points for LAr cryostat, ATLAS note ATL-AB-UR-0057, may 2004.

ATLAS, Position of the LAr barrel in the tile barrel 64 modules after adjustement, Measurements of week 02 of 2005, ATLAS note ATL-LB-UR-0055, january 2005.

[25] H. Wilkens, on behalf of the ATLAS-LAr electronics group, First Experience with the Readout Electronics on the ATLAS EM Barrel Calorimeter at Cold, to be published in Nuclear Science Symposium and Medical Imaging Conference Record (IEEE2004).

[26] M. Chalifour et al, Studies for cooling down the ATLAS electromagnetic barrel calorimeter, ATLAS note EDMS ATL-AB-EN-0029, september 2003.

[27] ATLAS Electromagnetic Barrel Calorimeter Collaboration, Linearity and Resolution of a ATLAS Electromagnetic Barrel Calorimeter module in the CERN Electron Test-beam.

Study of the Response Uniformity on the Modules calibrated with an Electron Beam.

Characterization of the ATLAS Electromagnetic Calorimeters with muons. papers to be submitted to NIM.

[28] B. Aubert et al., Nucl. Instrum. Methods A 500 (2003) 202. 


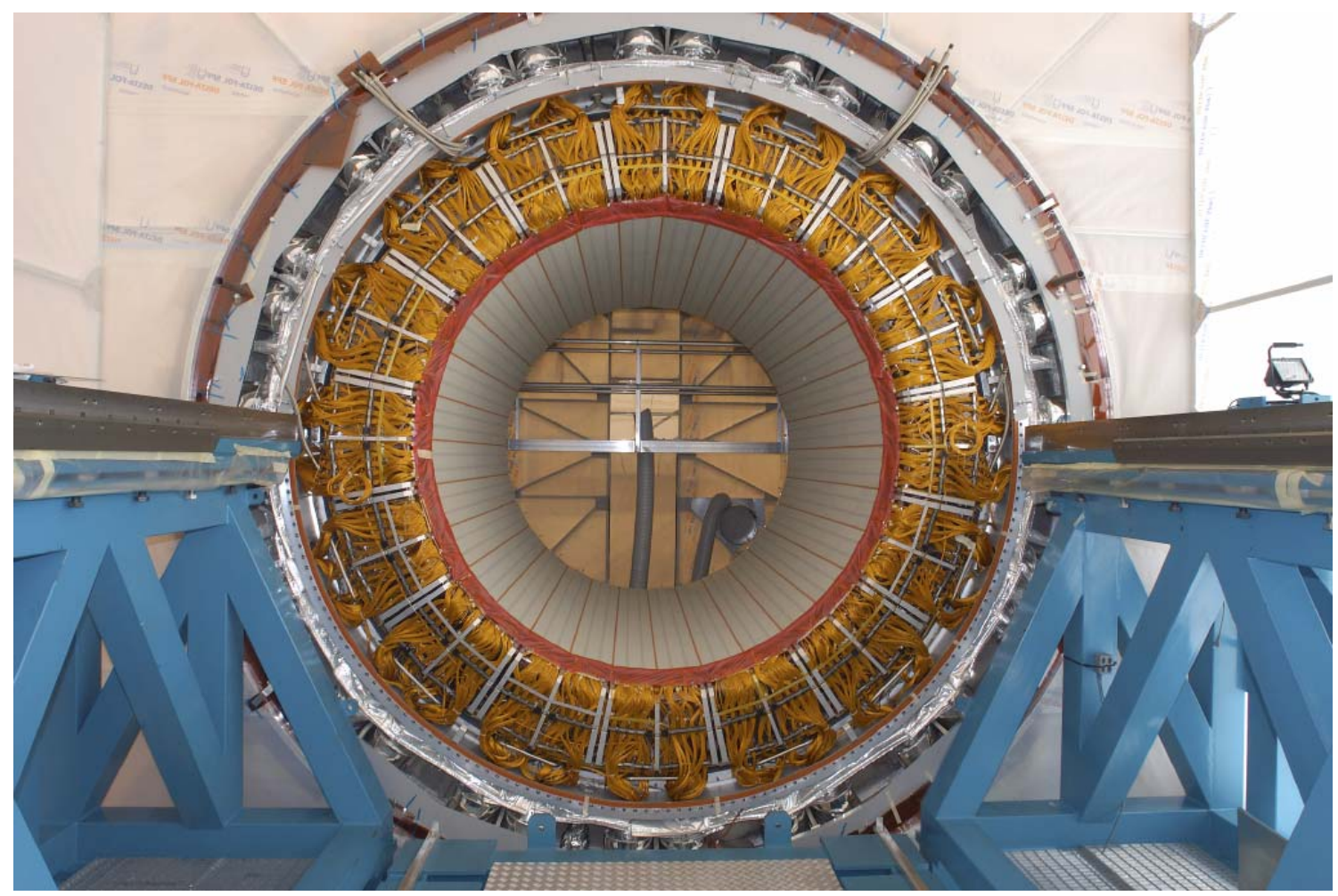

Fig. 1. First half barrel inserted inside the ATLAS cryostat. 


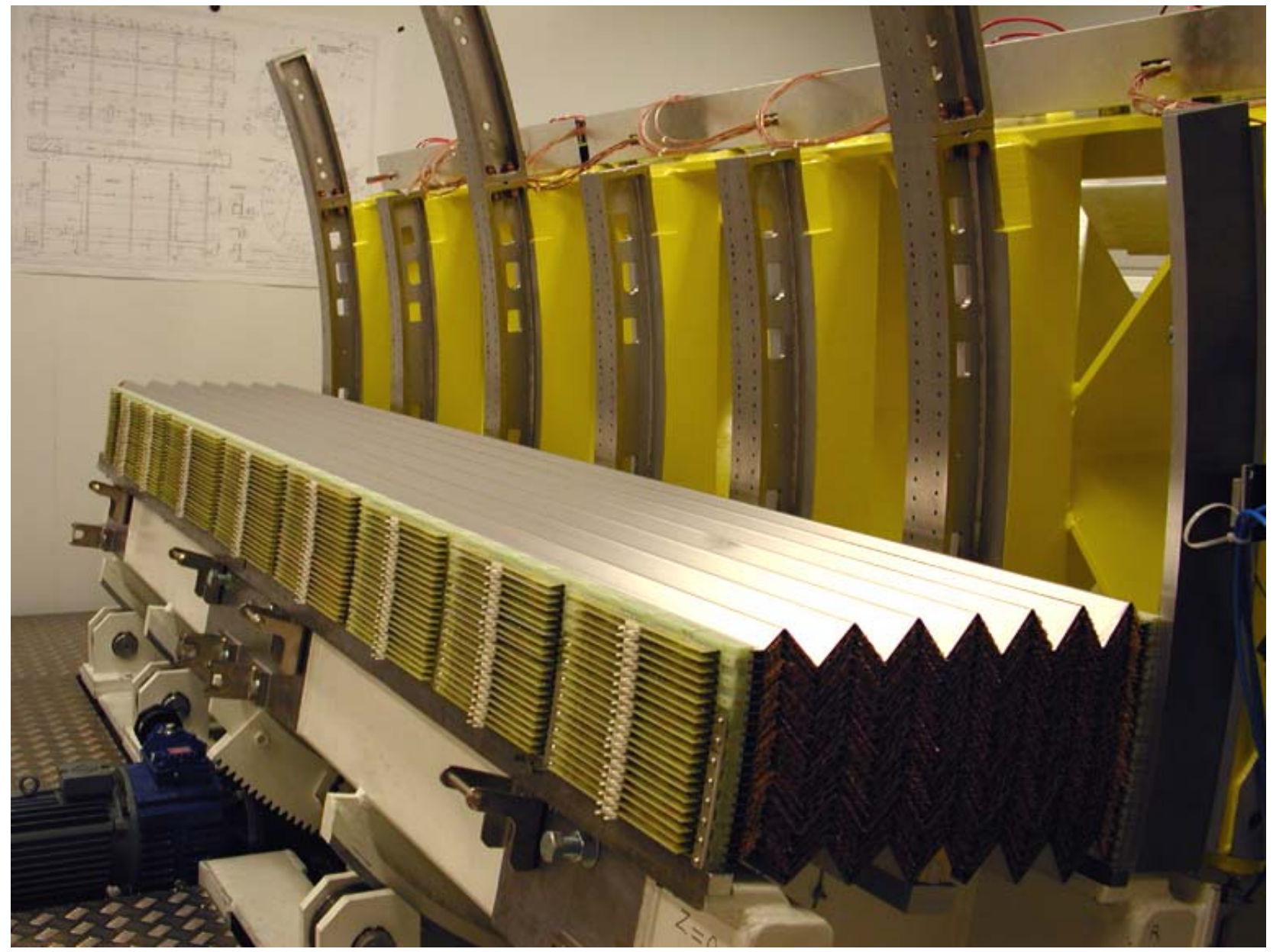

Fig. 2. One partly stacked module. The 6 outer rings on which are screwed the absorbers can be seen. Also the backbone (yellow part) and the assembling bench (white part) are visible. 

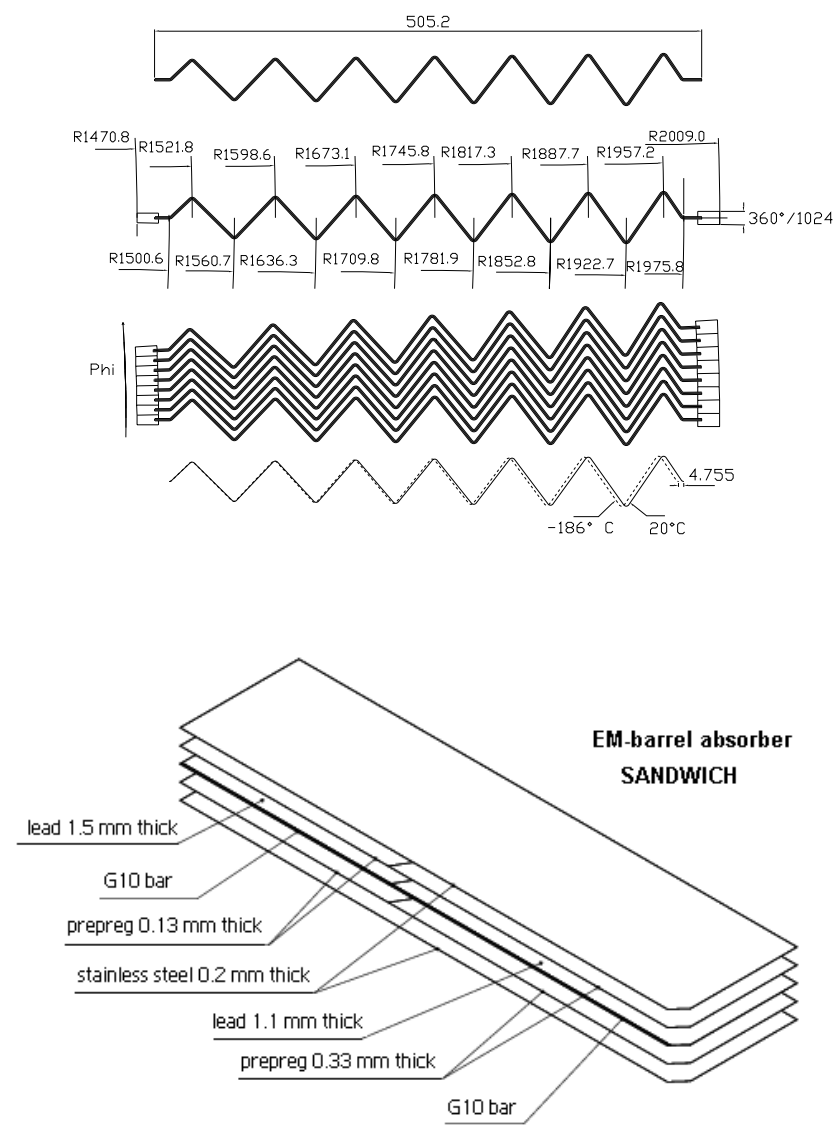

Fig. 3. The upper figure shows the absorber profile and a sketch of a stack of 5 absorbers. The lower figure shows the piling up of the absorber sandwich with the 2 lead thicknesses, before folding the absorber in an accordion shape. 

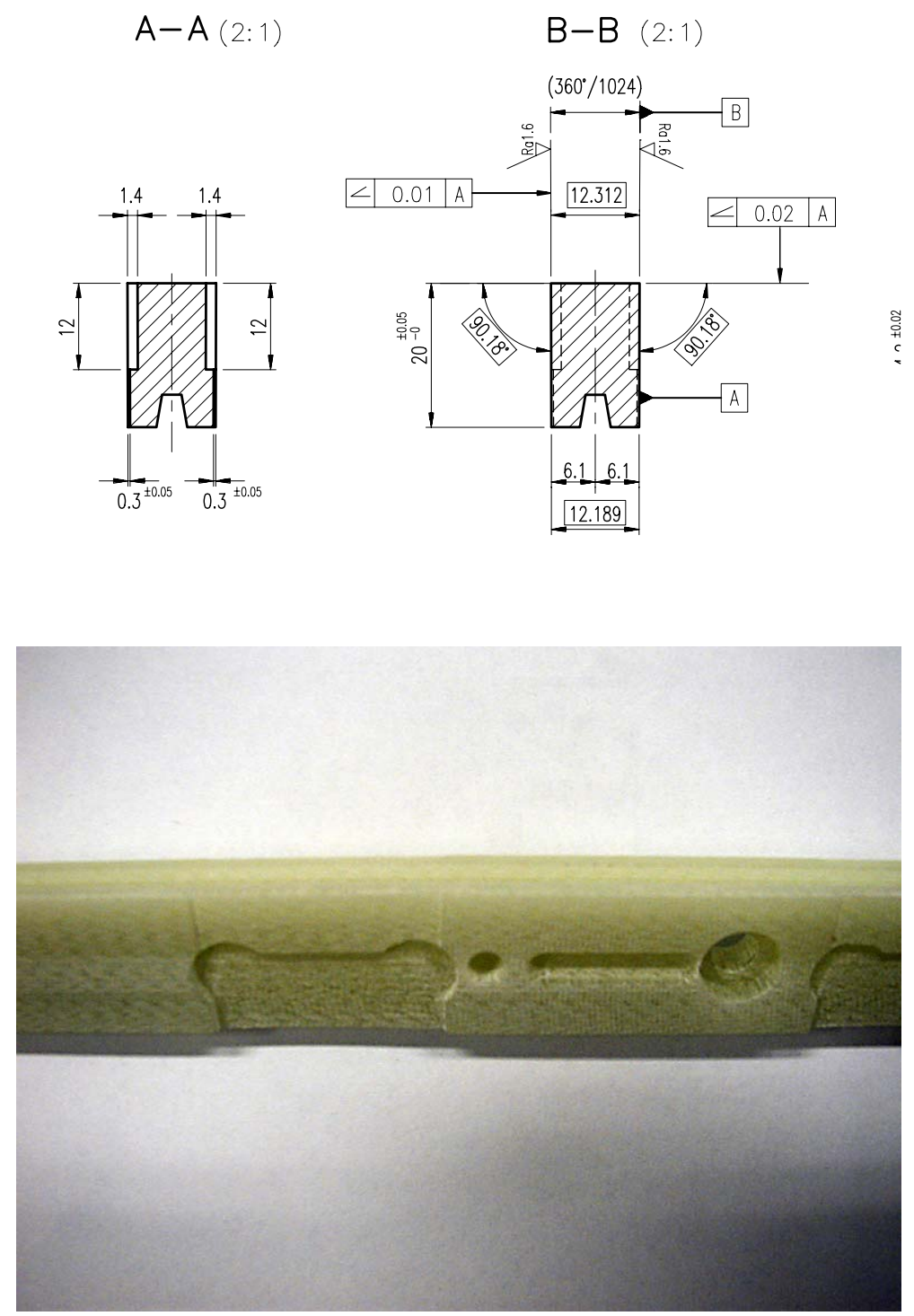

Fig. 4. G10 bar: transverse view drawing showing the $2 \pi / 1024$ angle and picture of a bar section (10 cm long) showing holes for dowel pins and cut-outs for connectors. 

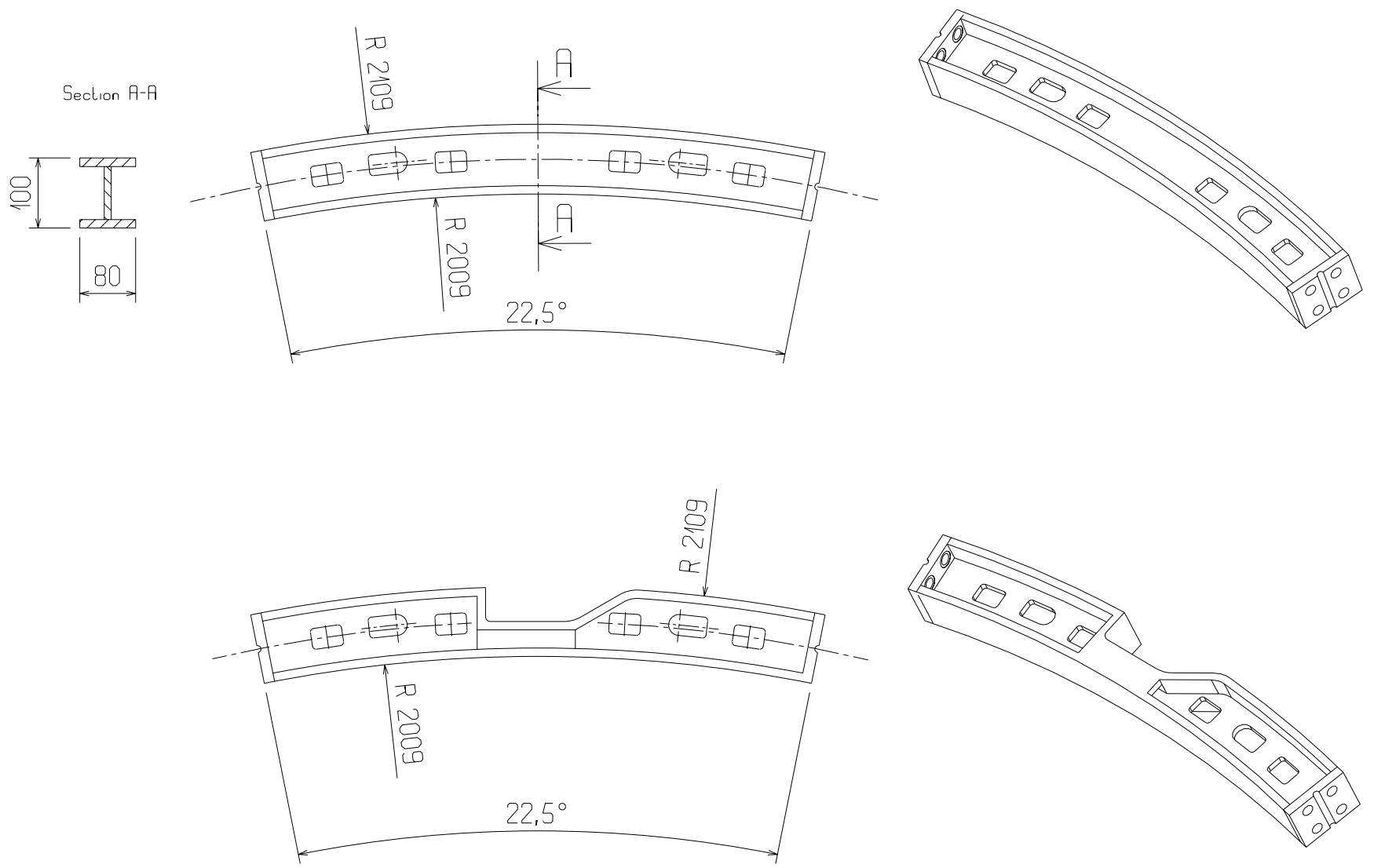

Fig. 5. Upper drawings : standard ring-piece ; lower drawings : ring-piece at the level of the cryostat rail.

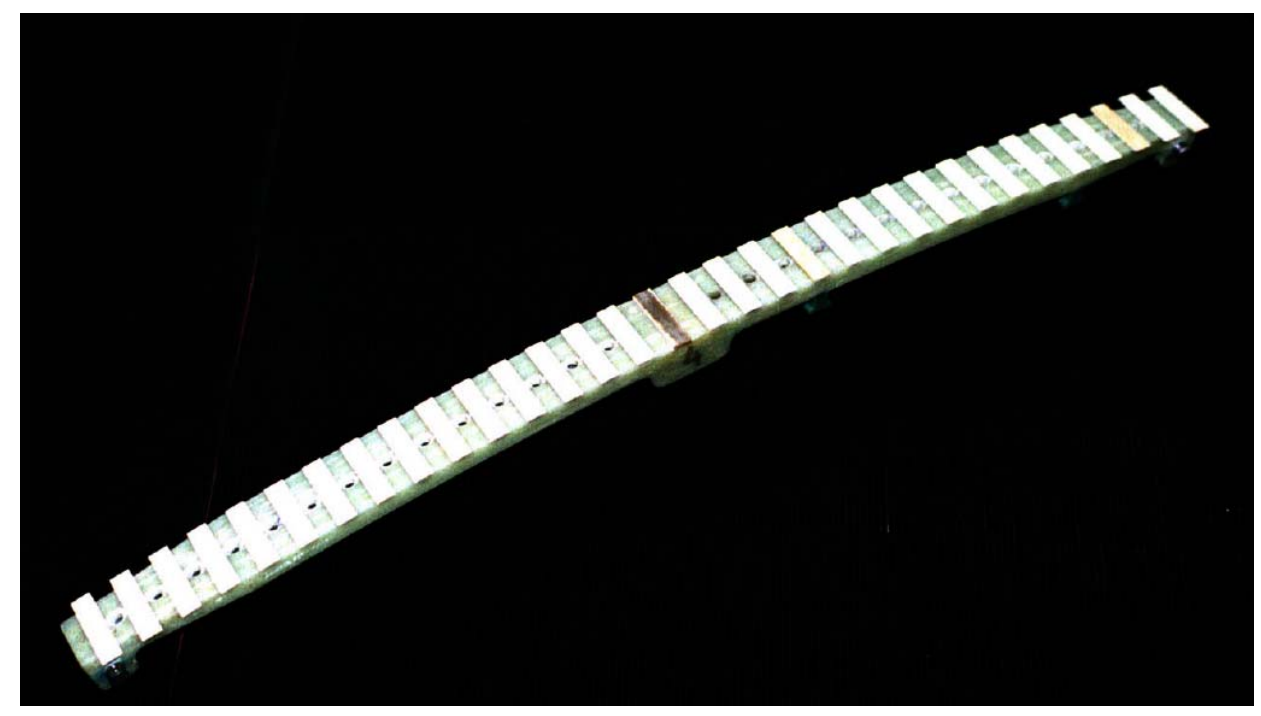

Fig. 6. Inner ring picture. 

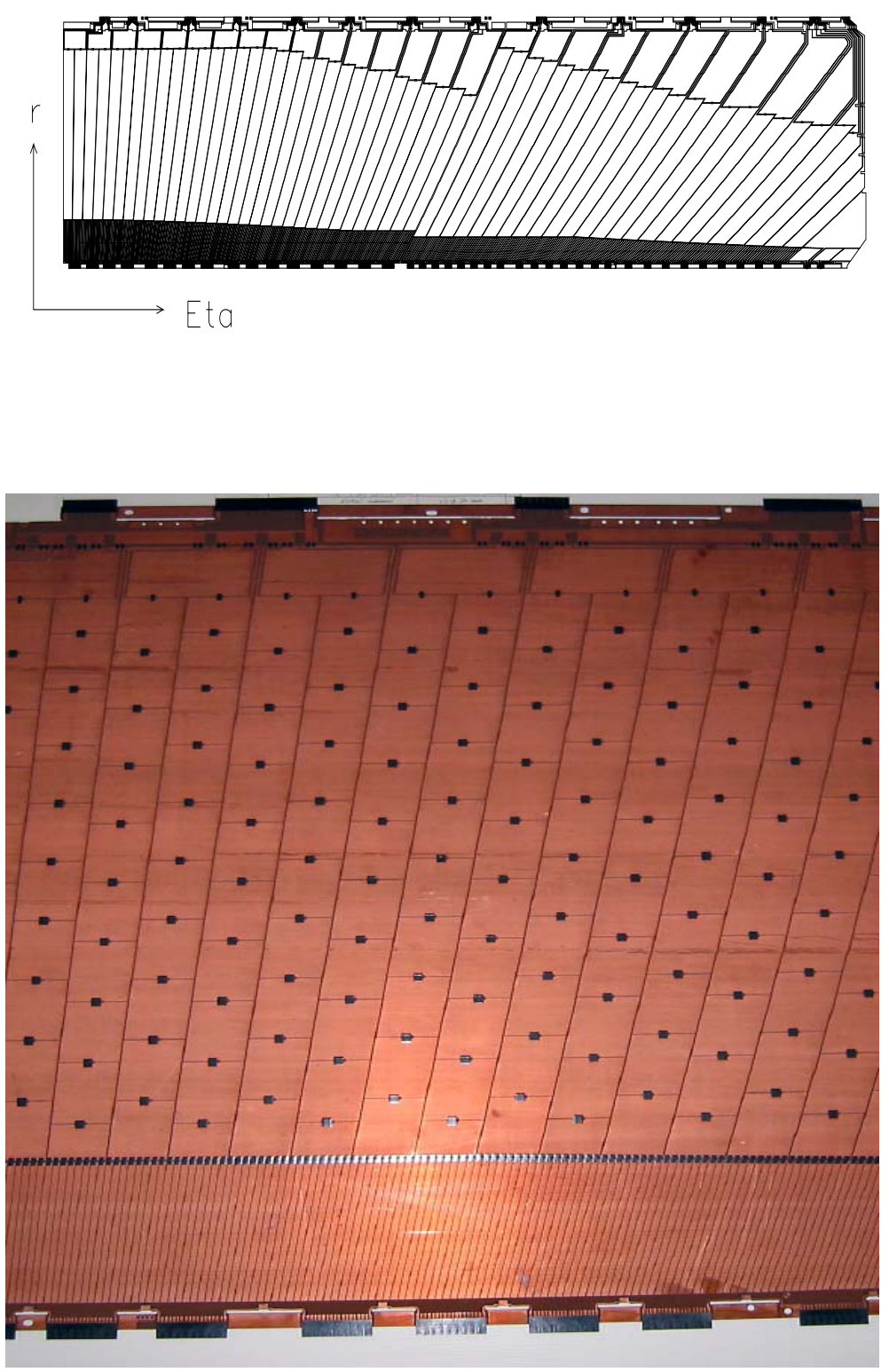

Fig. 7. Upper part: etched pattern, before folding, on A+B electrodes signal layer. Lower part: picture of a section, about $50 \mathrm{~cm}$ long, of an A electrode, before folding, showing the etched pattern of the HT layer where the resistive silk-screened pads of carbon-loaded epoxy ink can easily be seen. (The copper strips between connectors have not yet been removed.) 


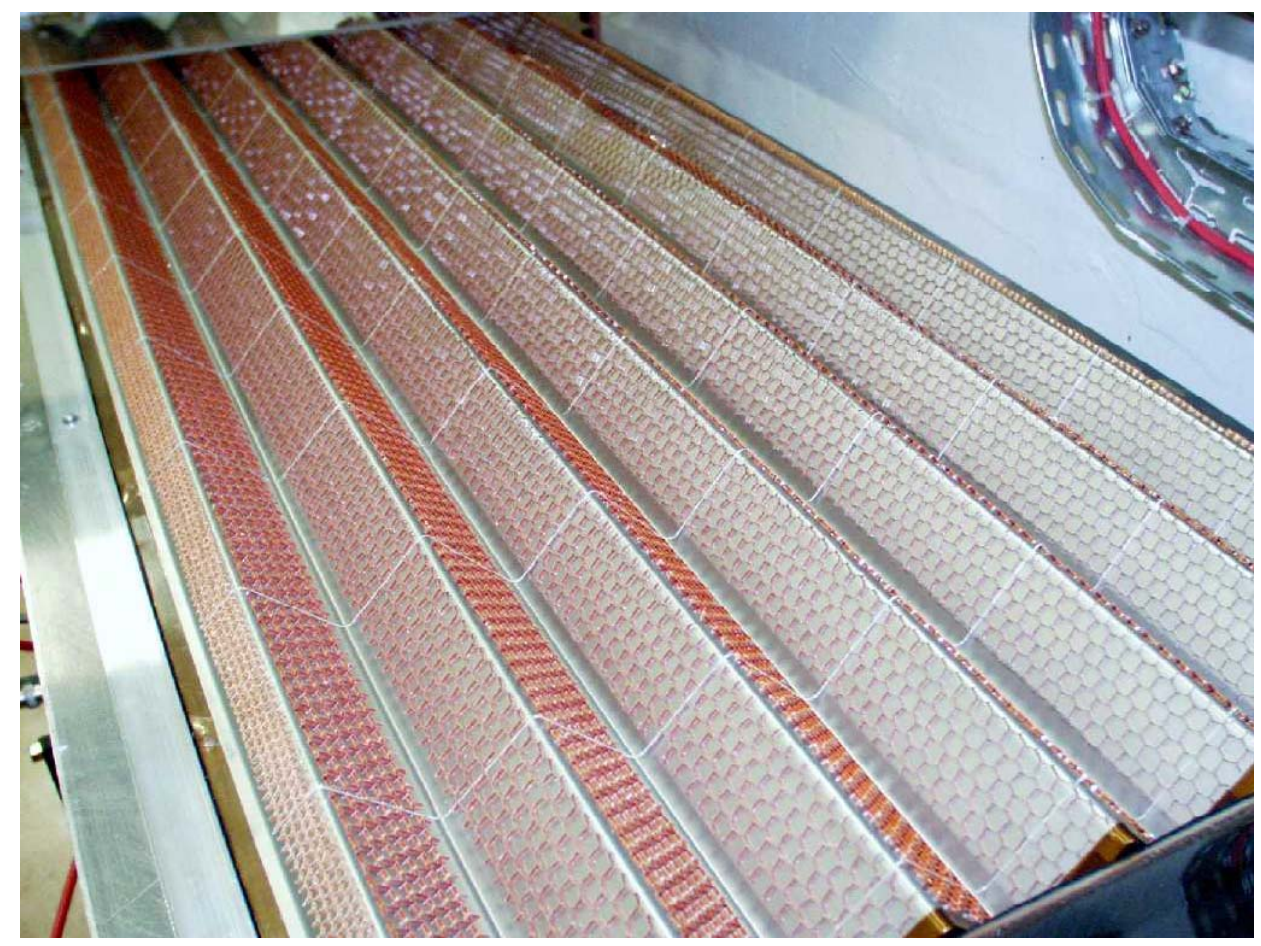

Fig. 8. One spacer resting on one absorber.

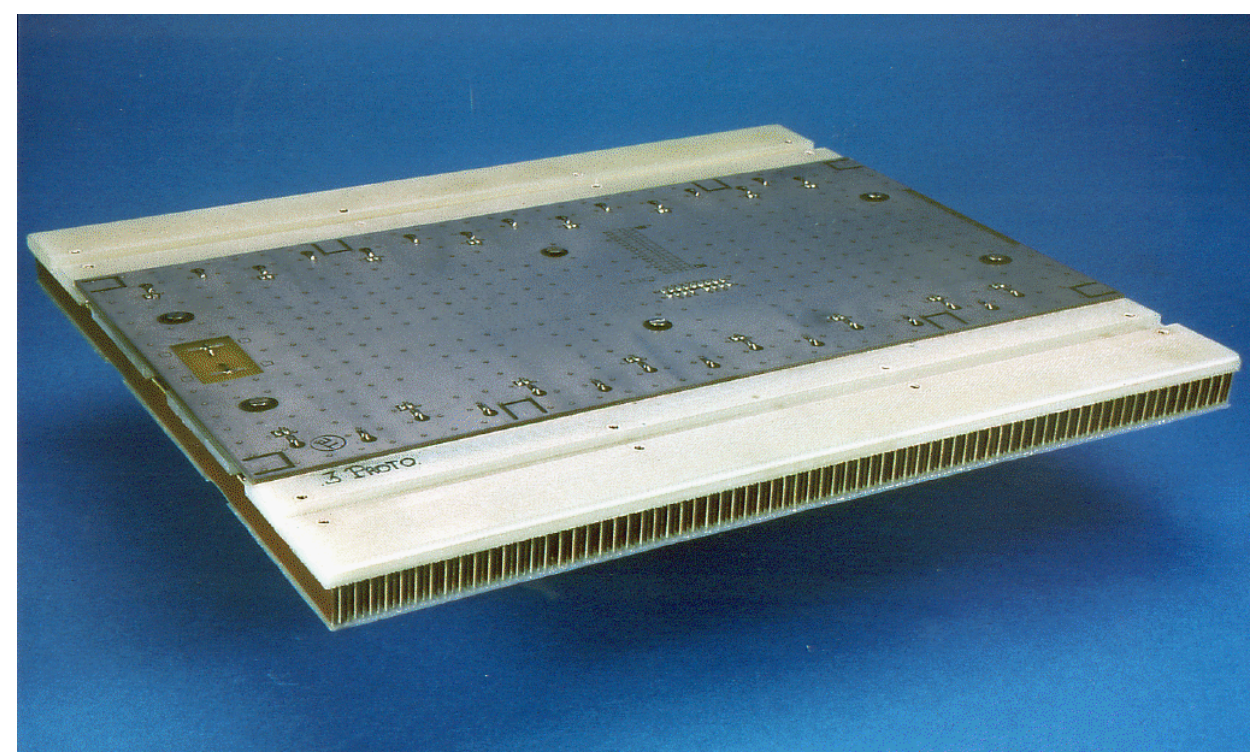

Fig. 9. A presampler module with its motherboard. 

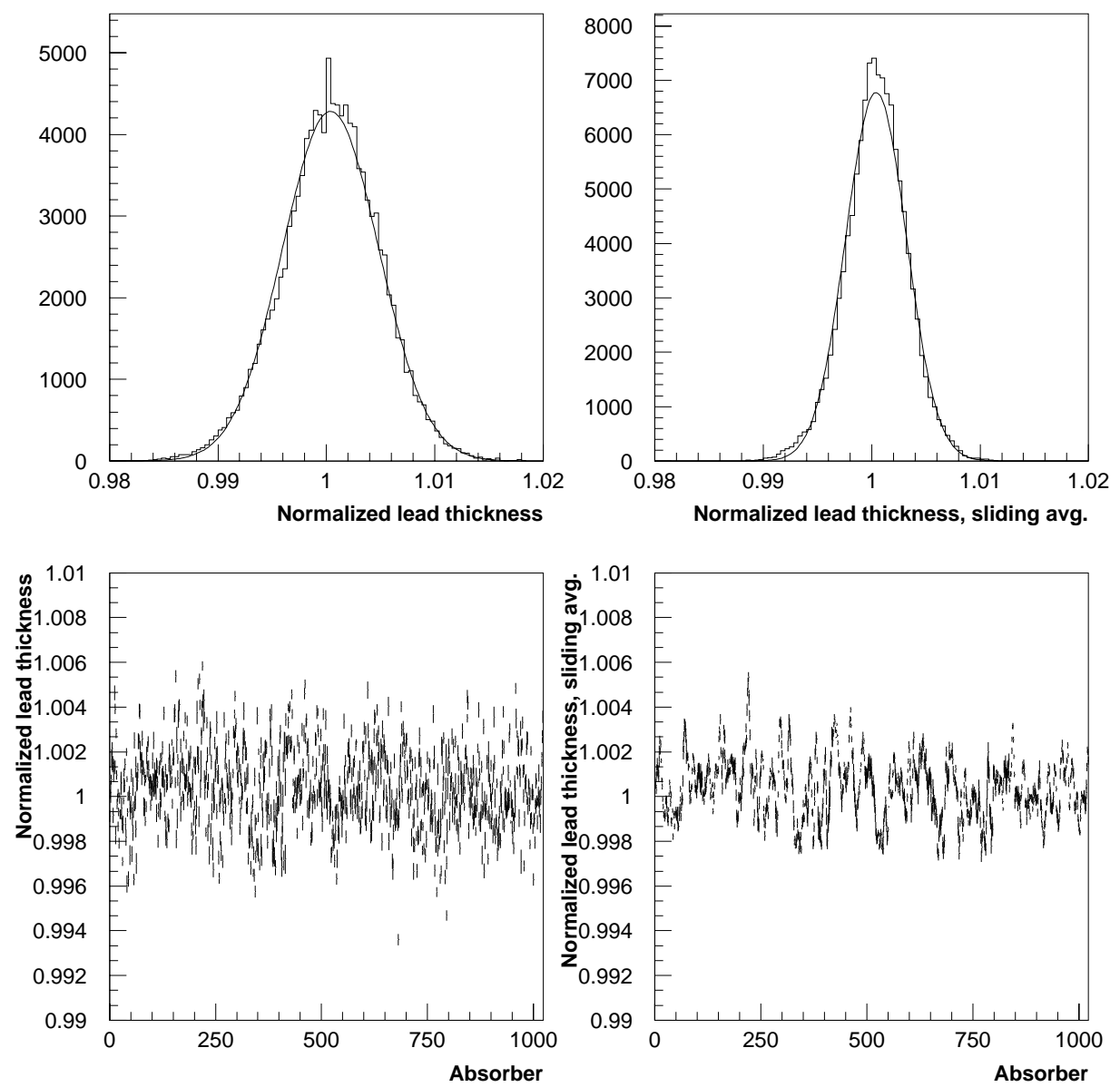

Fig. 10. Upper plots : Distribution of the normalized lead thickness for all the $\eta$ cells of all the absorbers of the barrel calorimeter. The effect of optimizing the arrangement of consecutive absorbers can be seen in the reduction of the rms of the sliding average, $0.31 \%$ (right upper plot) instead of $0.44 \%$. The thickness for each middle sampling cell is calculated by taking the average of all the measurements on the lead plate, that fall within the corresponding cell $\eta$ range. From the r.m.s. of the distribution of the sliding average of the normalized thickness over 5 consecutive absorbers (upper right plot), it is possible to deduce that the contribution of the residual lead thickness inhomogeneities to the constant term $[9]$ is $0.31 \% \times 0.6=0.19 \%$. The normalized lead thickness as a function of $\phi$ or absorber number shows that there are no systematic trends (lower plots). 


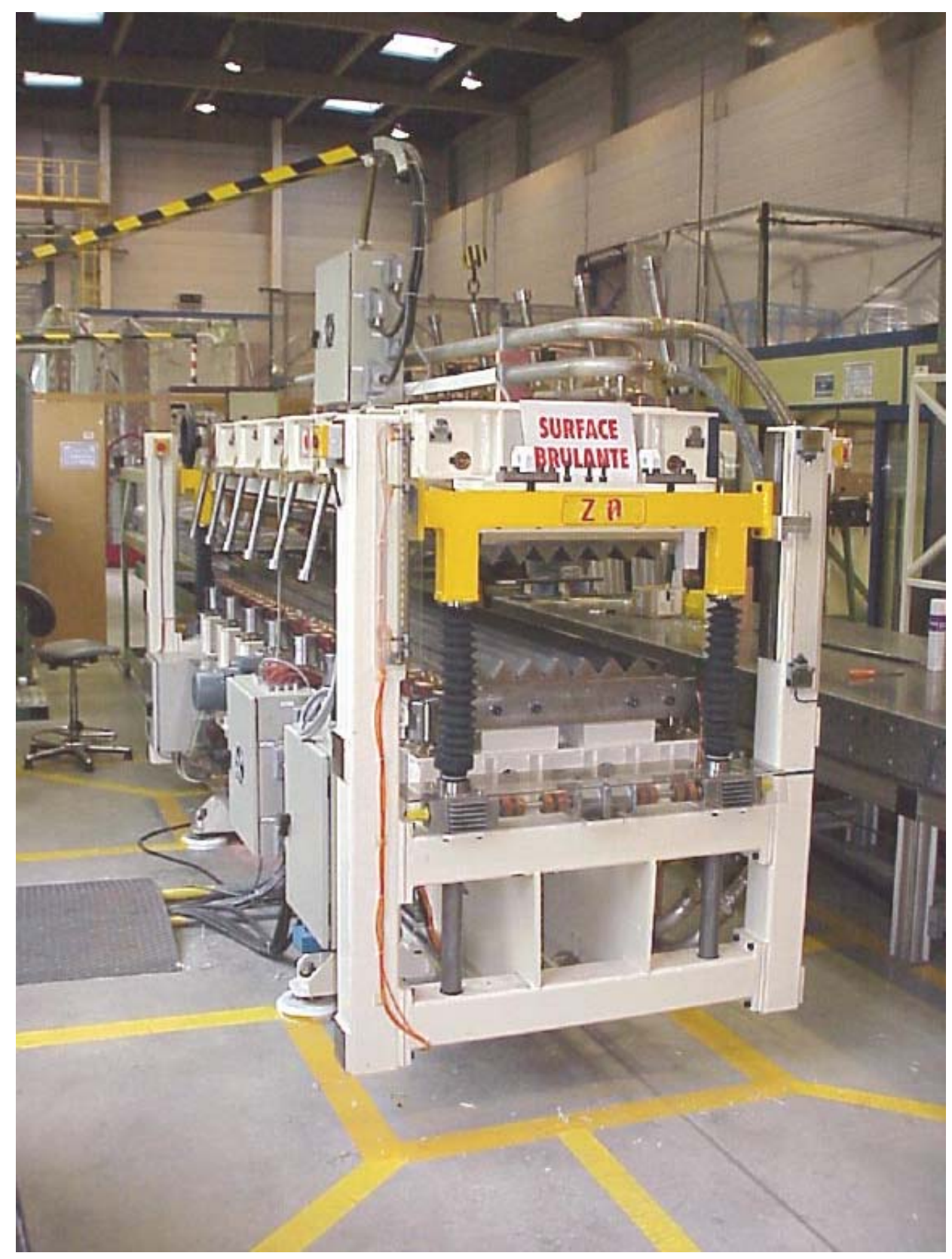

Fig. 11. Picture of the heating press where the absorber is placed for curing after folding. 

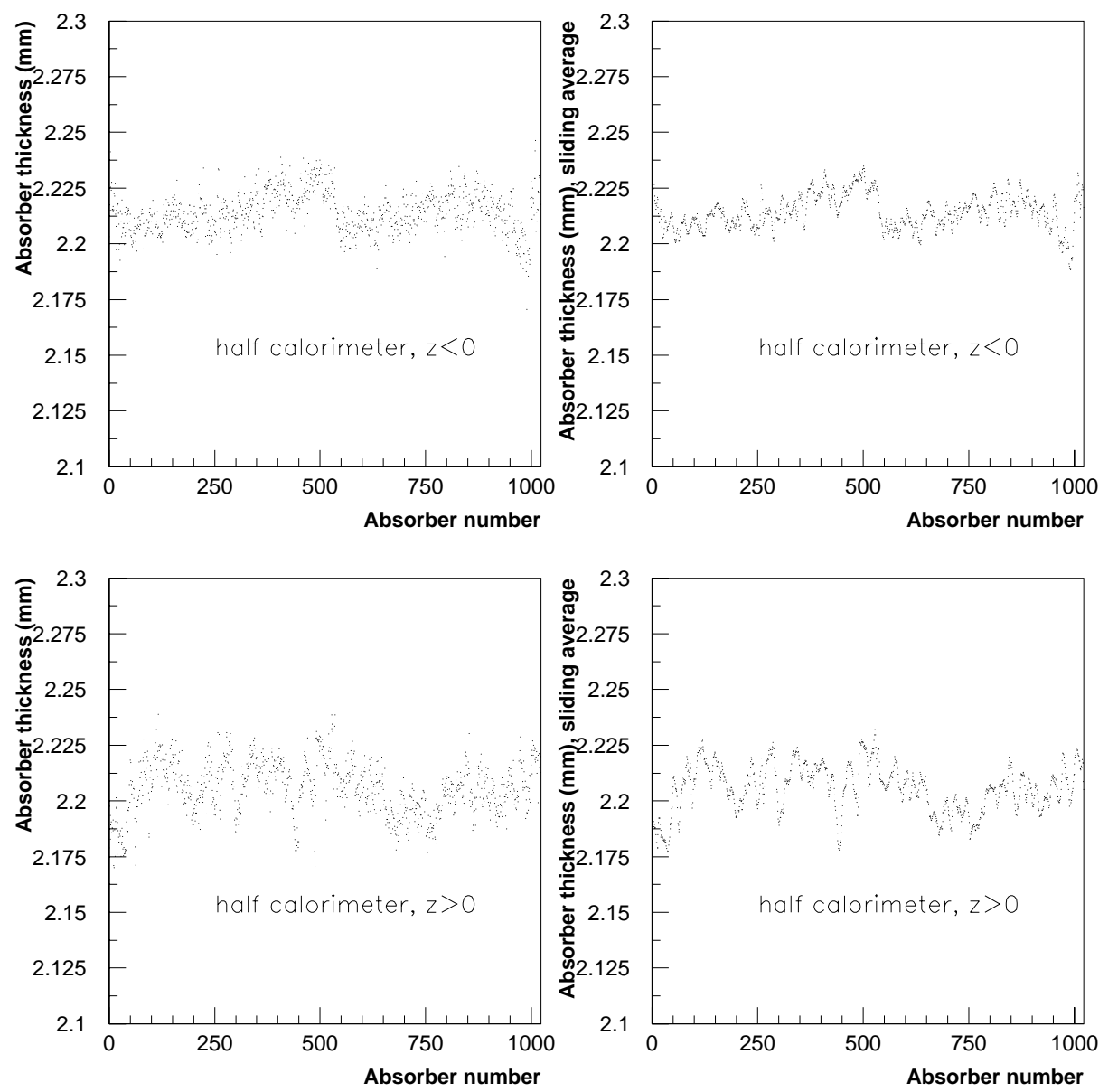

Fig. 12. Distribution of the absorber mean thickness (left plots), and of the thickness averaged over 5 consecutive absorbers (right plots) versus $\phi$ for the 2 half calorimeters. The stability of the production chain is at the level of $1 \%$. 

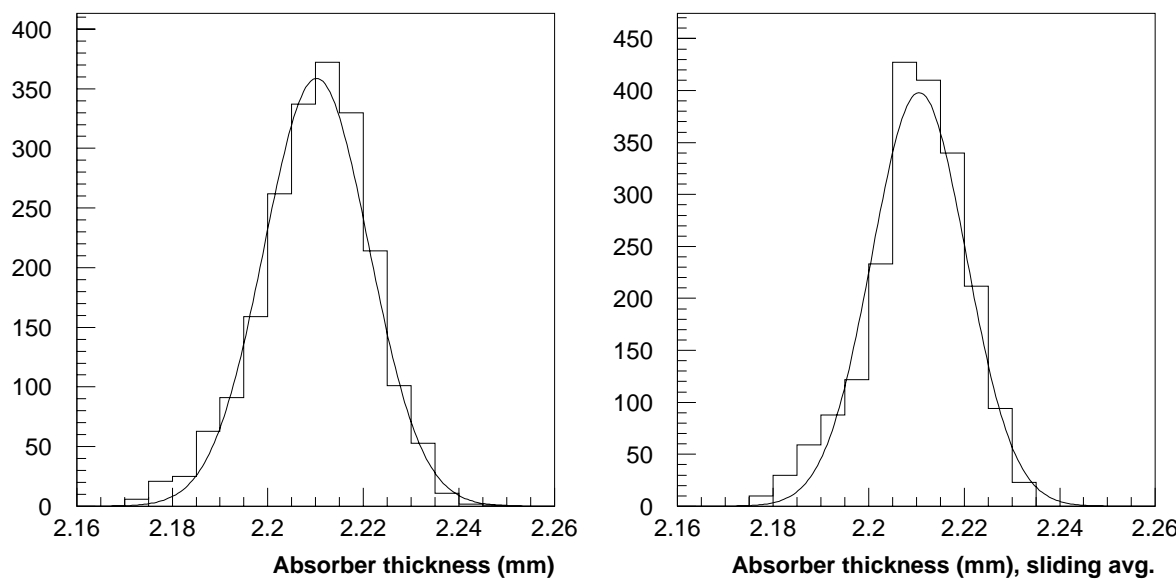

Fig. 13. The left plot shows the distribution of the absorber thickness and the right plot shows the distribution of the absorber thickness sliding average. The mean thickness is $2.21 \mathrm{~mm}$, and the $\mathrm{rms}$ are respectively $0.011 \mathrm{~mm}$ and $0.010 \mathrm{~mm}$. As for the lead, the r.m.s. of the distribution of the sliding average of the thickness of 5 consecutive absorbers (right plot), is used to give an estimation of the corresponding contribution to the constant term, as being this r.m.s. divided by the distance between two absorbers and multiplied by a numerical coefficient $(0.3)$ due to the drift velocity dependence on the electrical field [10]. This gives a contribution to the constant term of $0.07 \%$. 


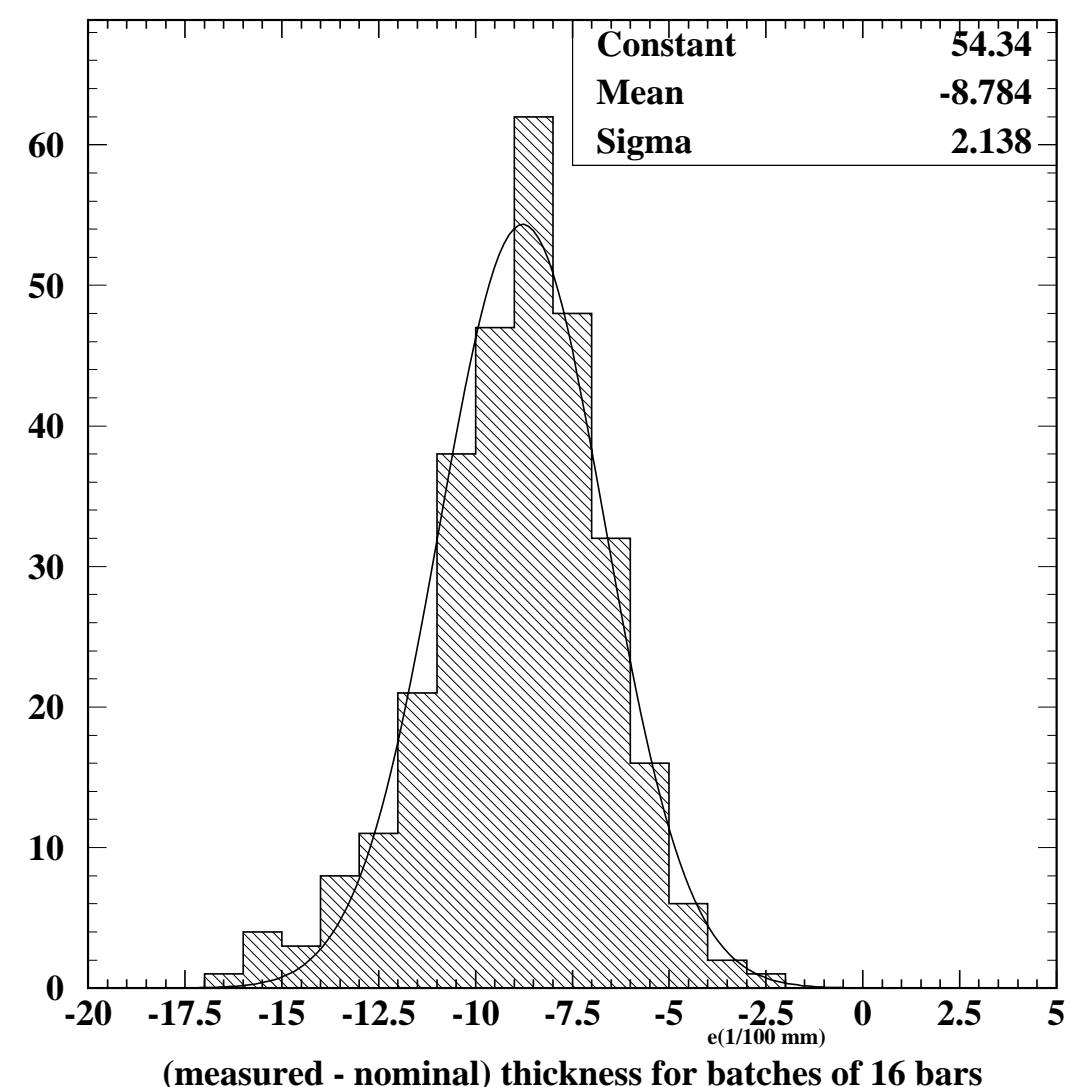

Fig. 14. Difference between actual and nominal thickness for 30 batches of 16 external bars and 30 batches of 16 internal bars. On average the batches are a bit too thin (average $-0.09 \mathrm{~mm}$, rms $0.02 \mathrm{~mm}$ ). 

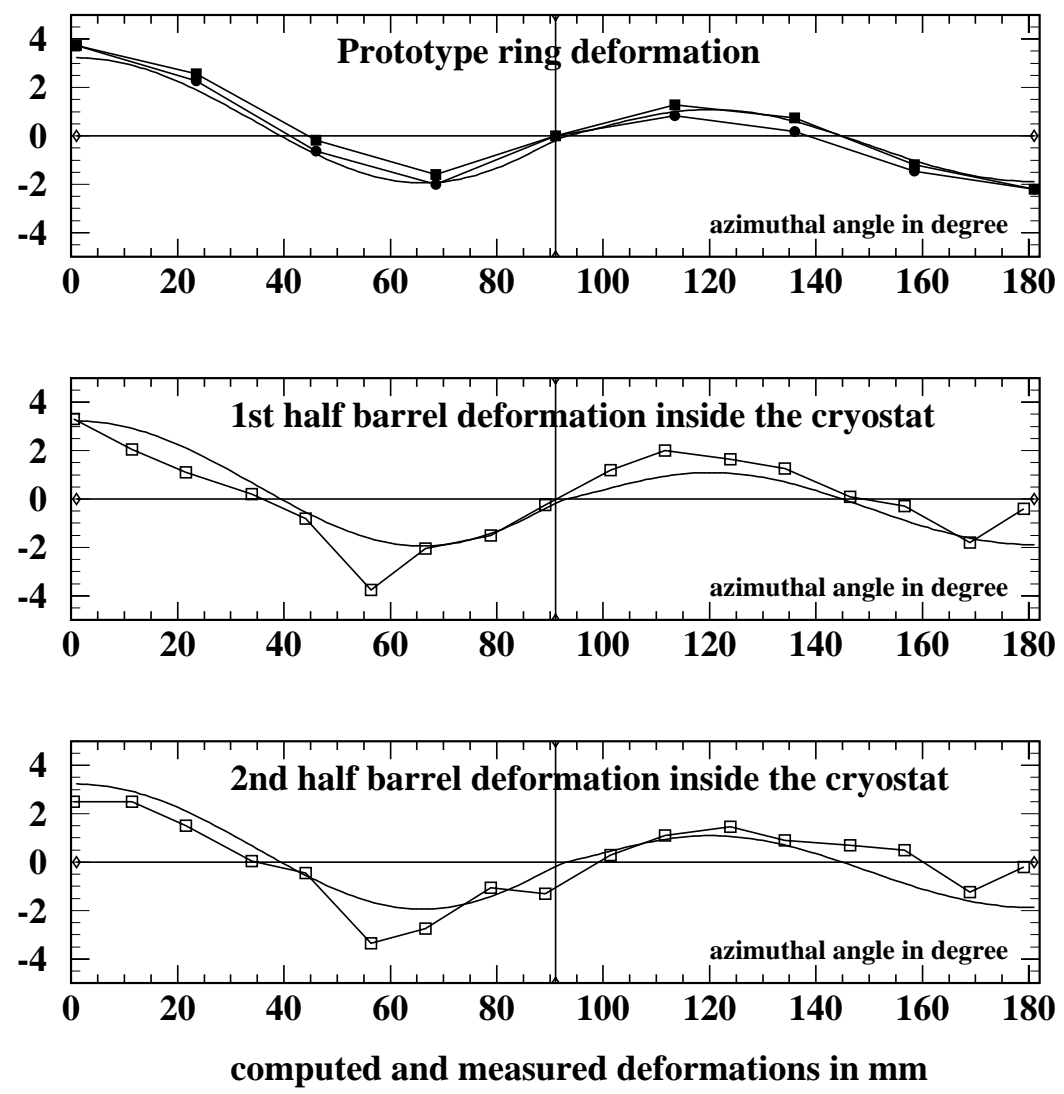

Fig. 15. The upper plot shows the radial deformation in mm under load of the prototype ring as a function of the azimuthal angle $\phi . \phi=0^{\circ}$ and $\phi=180^{\circ}$ correspond respectively to the bottom and top ring-pieces and $\phi=90^{\circ}$ to the 2 support ring-pieces. A positive (negative) value means an increase (a decrease) of the nominal ring radius. Black dots and black squares correspond respectively to the left and right side of the ring. The continuous curve is the result of a finite element calculation. The 2 lower plots show the deformation of each half barrel at $|z|=3200 \mathrm{~mm}$ and at the level of the external ring, once inserted in the cryostat. The open squares show the average deformation between the left and right side of the barrel. 


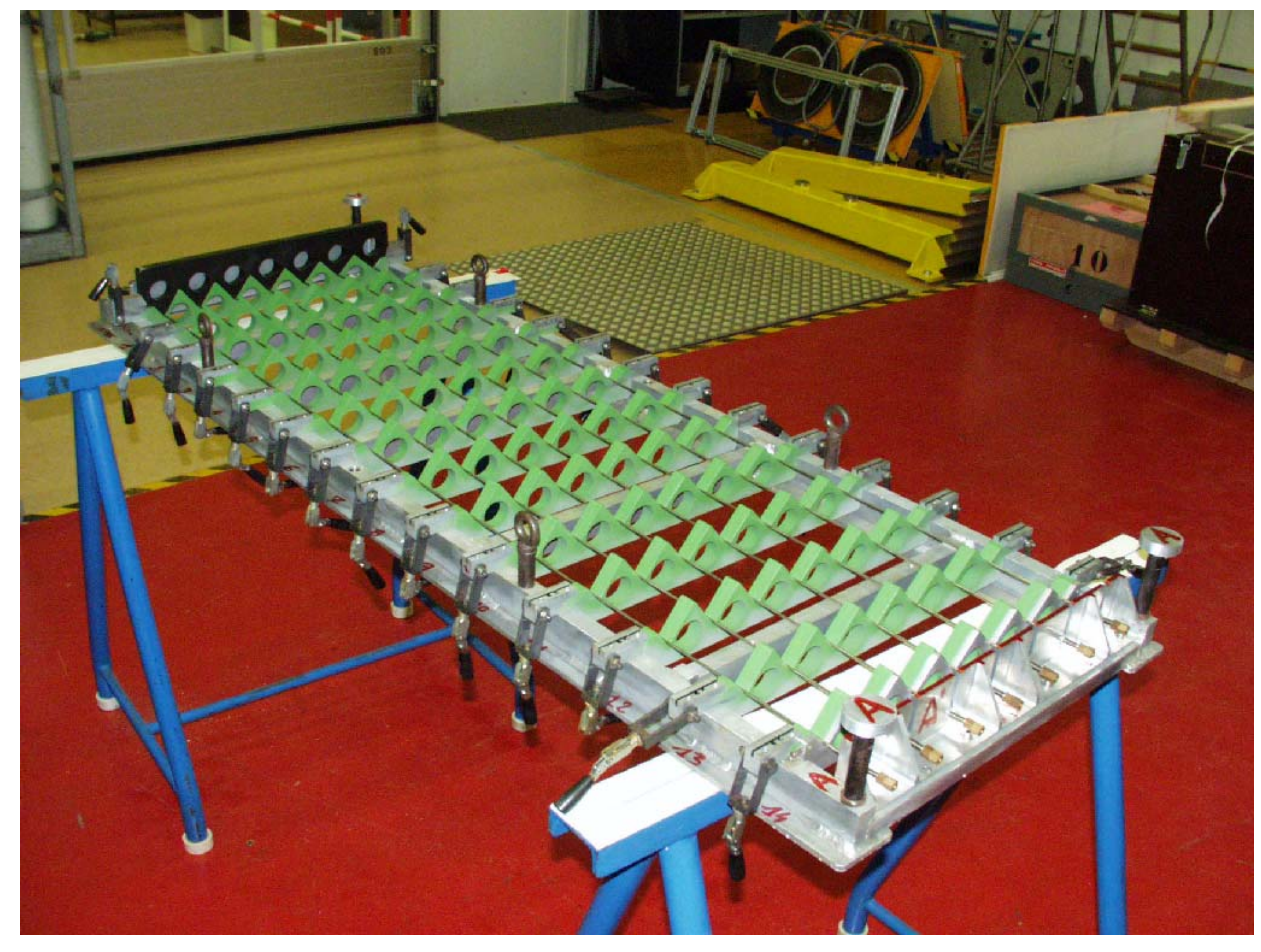

Fig. 16. Tooling used to produce a spacer. 

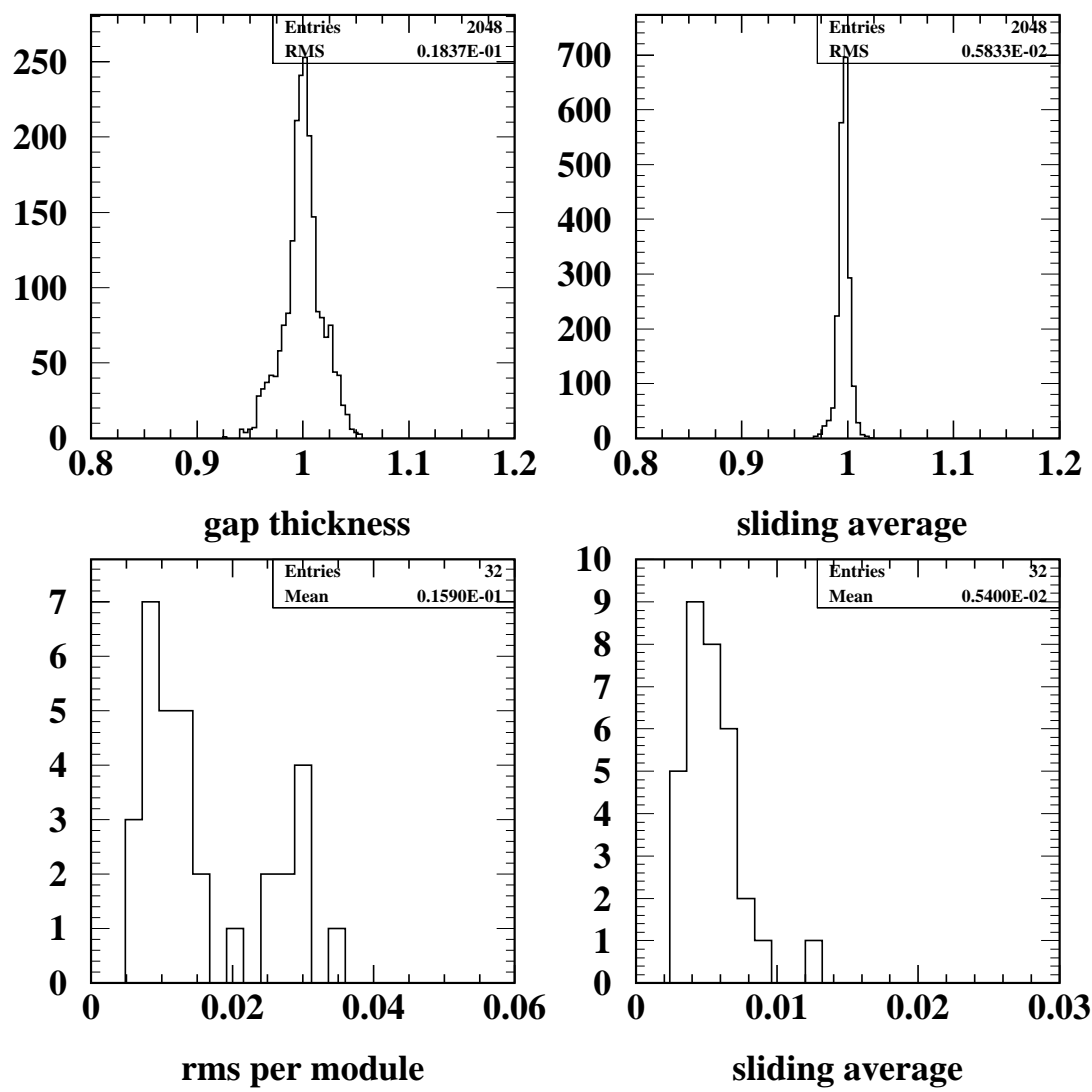

Fig. 17. The top left plot shows the normalized gap thickness deduced from the gap capacity mesurements. The top right plot shows the same distribution using a sliding average over 5 consecutive gaps, quantity used to determine the contribution to the constant term. The bottom plots present the rms of the normalized gap thickness for each module. 

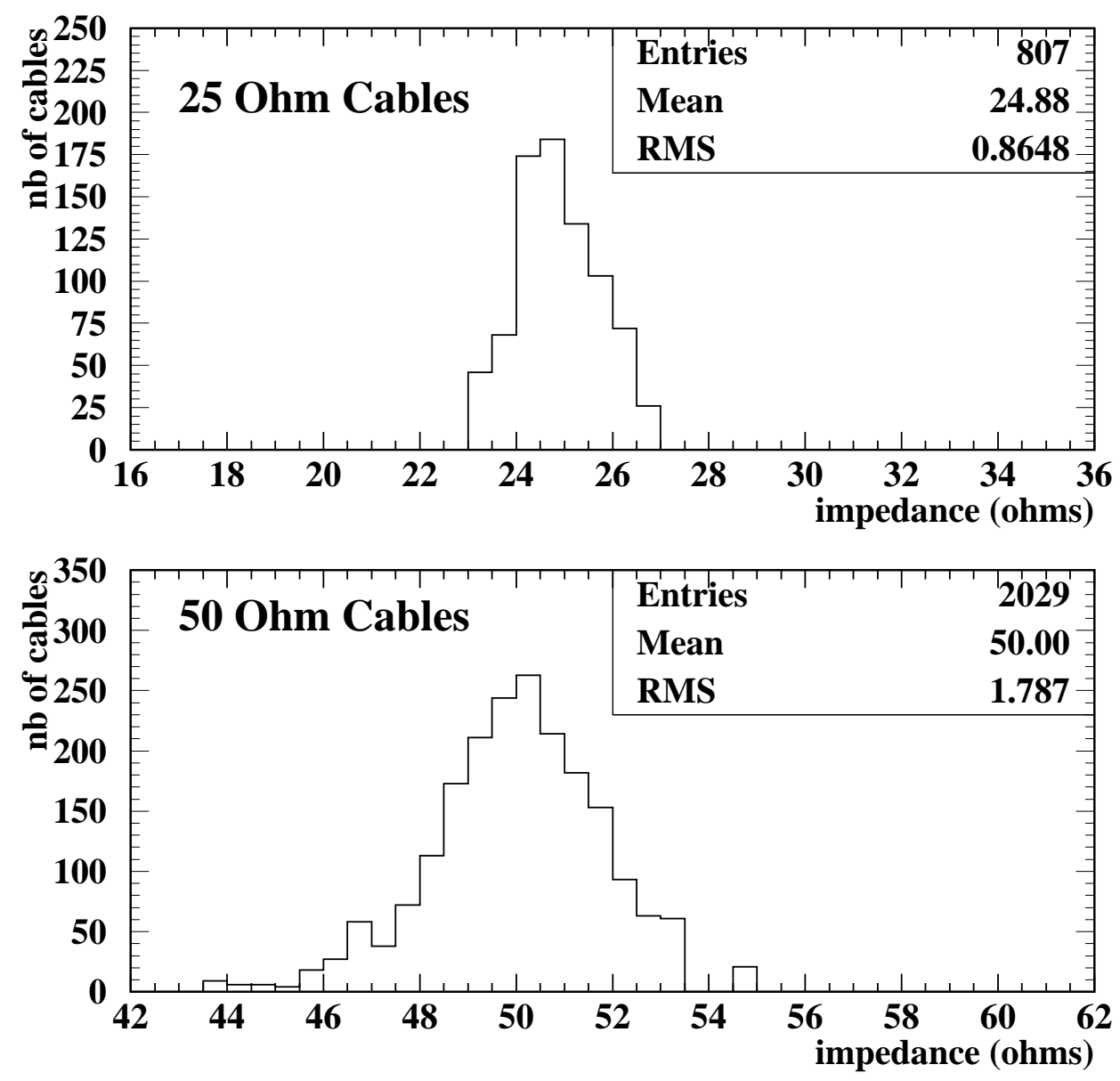

Fig. 18. Distribution of the impedances of the $25 \Omega$ and the $50 \Omega$ cables. This measurement was performed by cutting a $70 \mathrm{~cm}$ piece at the end of each $200 \mathrm{~m}$ long cable coil. 


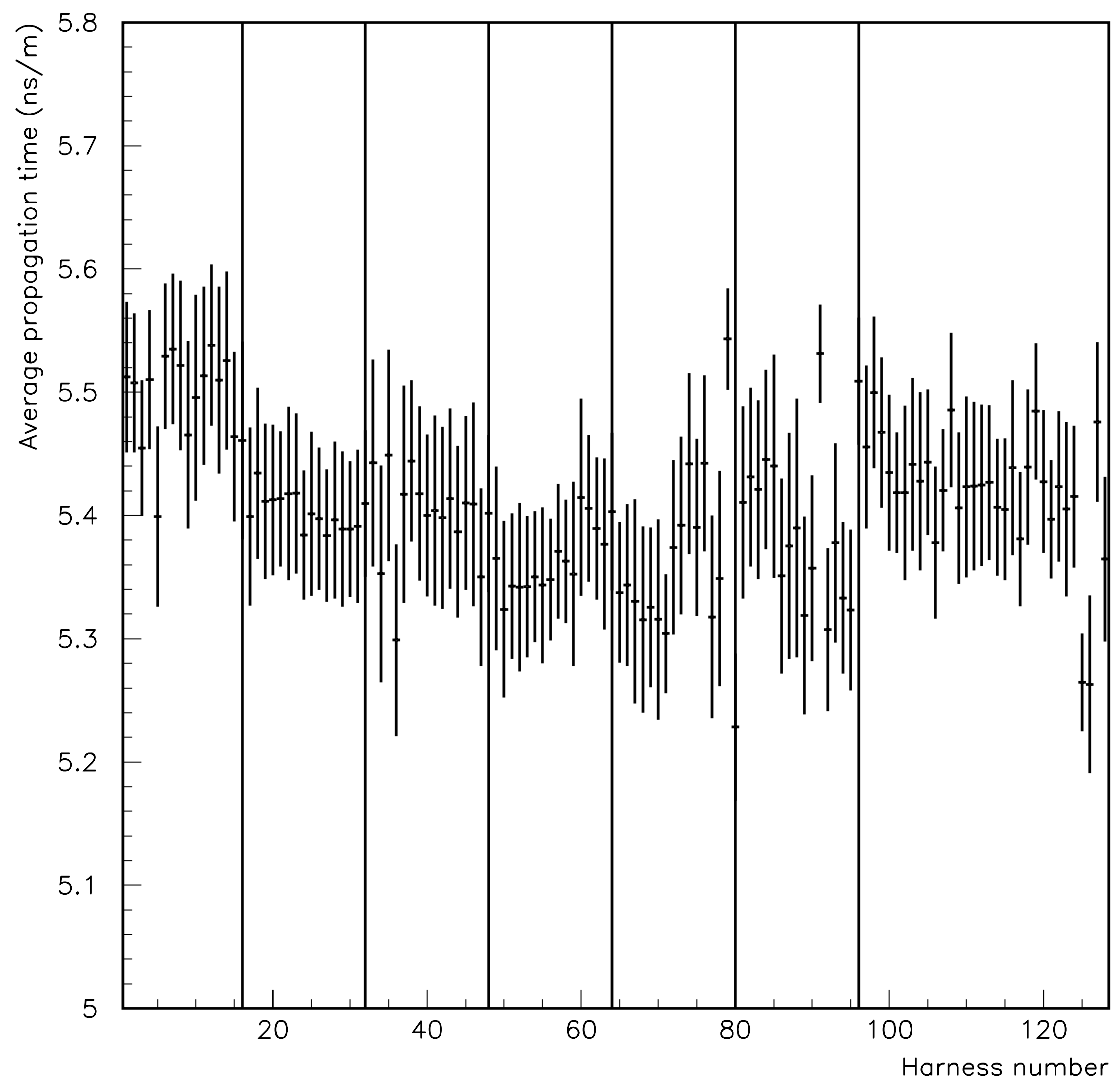

Fig. 19. Average and rms propagation time (per meter) of $4 \mathrm{~m}$ long 64-channel harnesses equipping the barrel from $\eta=0$ to $\eta=0.2$. The vertical lines separate different batches from the manufacturer. 


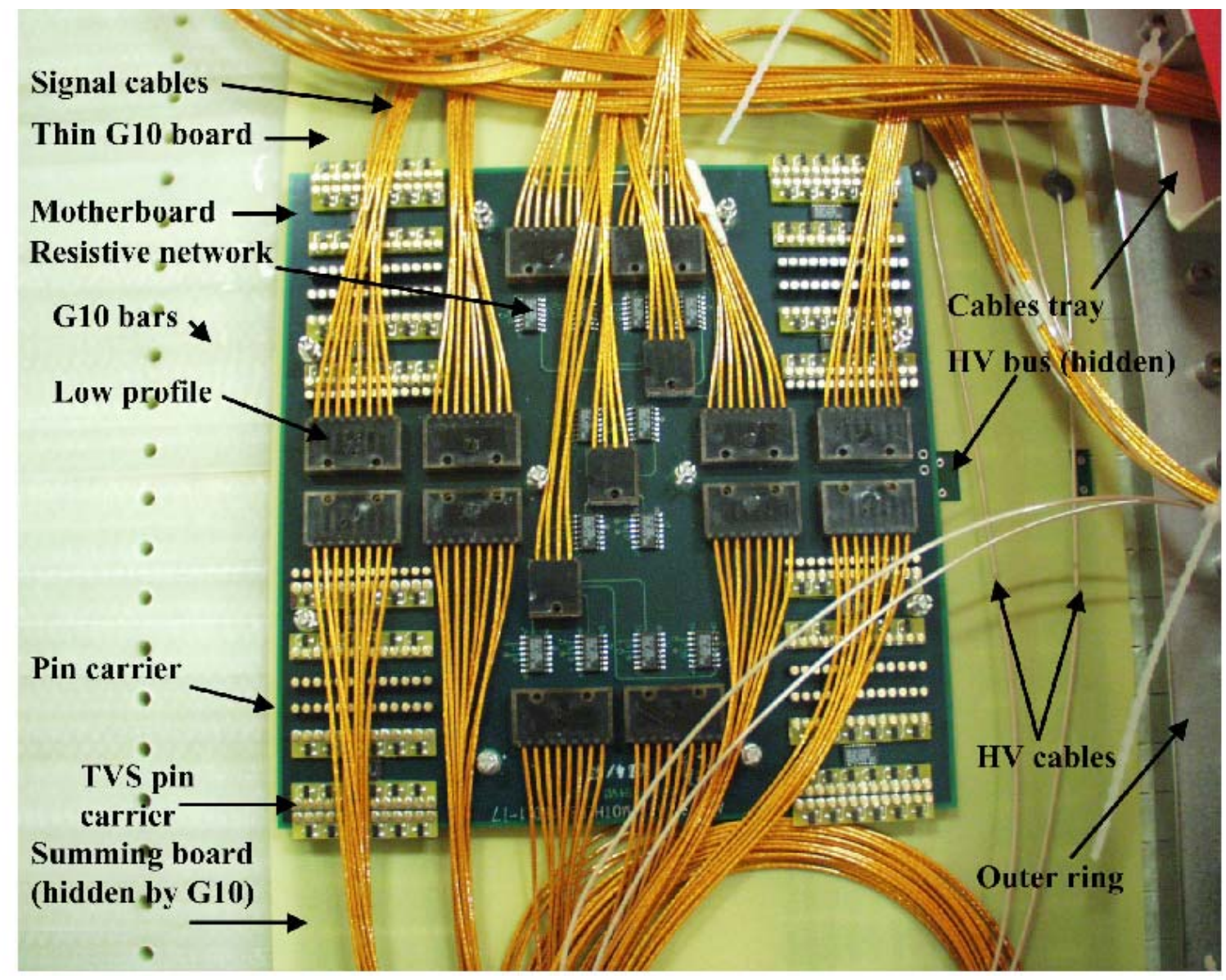

Fig. 20. Back mother board on a module after cabling. The summing boards are hidden by the thin G10 boards. 
Strip layer

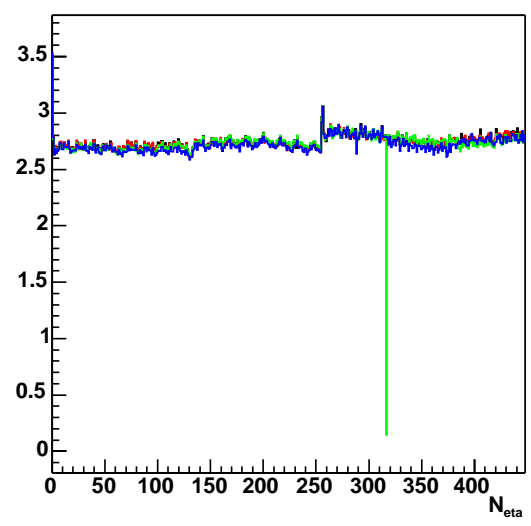

Back layer

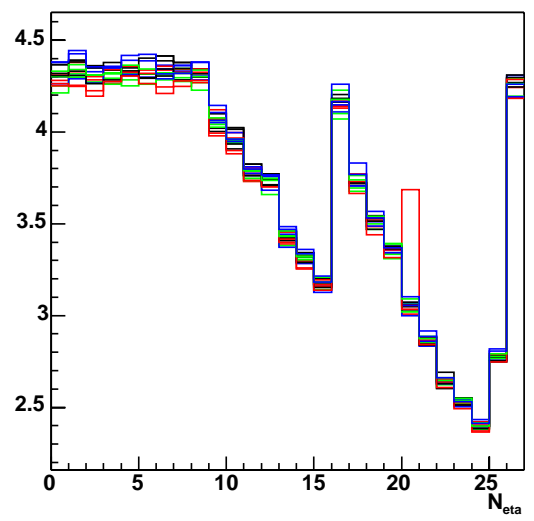

Middle layer

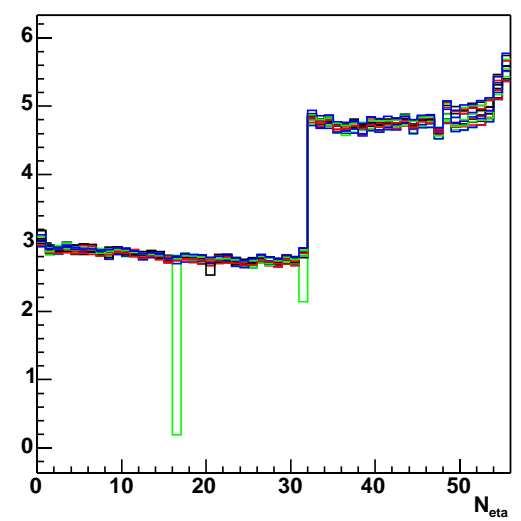

Barrel end

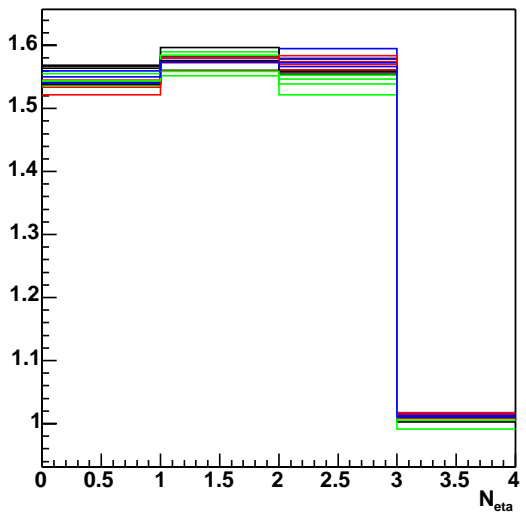

Fig. 21. Maximum, after shaping, of the TPA test signal as a function of the $\eta$ number for all the $\phi$ lines for one barrel module, for the three layers and the barrel-end. The shaped signal varies according to the detector capacitance. Several categories of problems can be seen here: channel with zero signal, with too fast or too slow rising time, corresponding to bad connections at different places. 


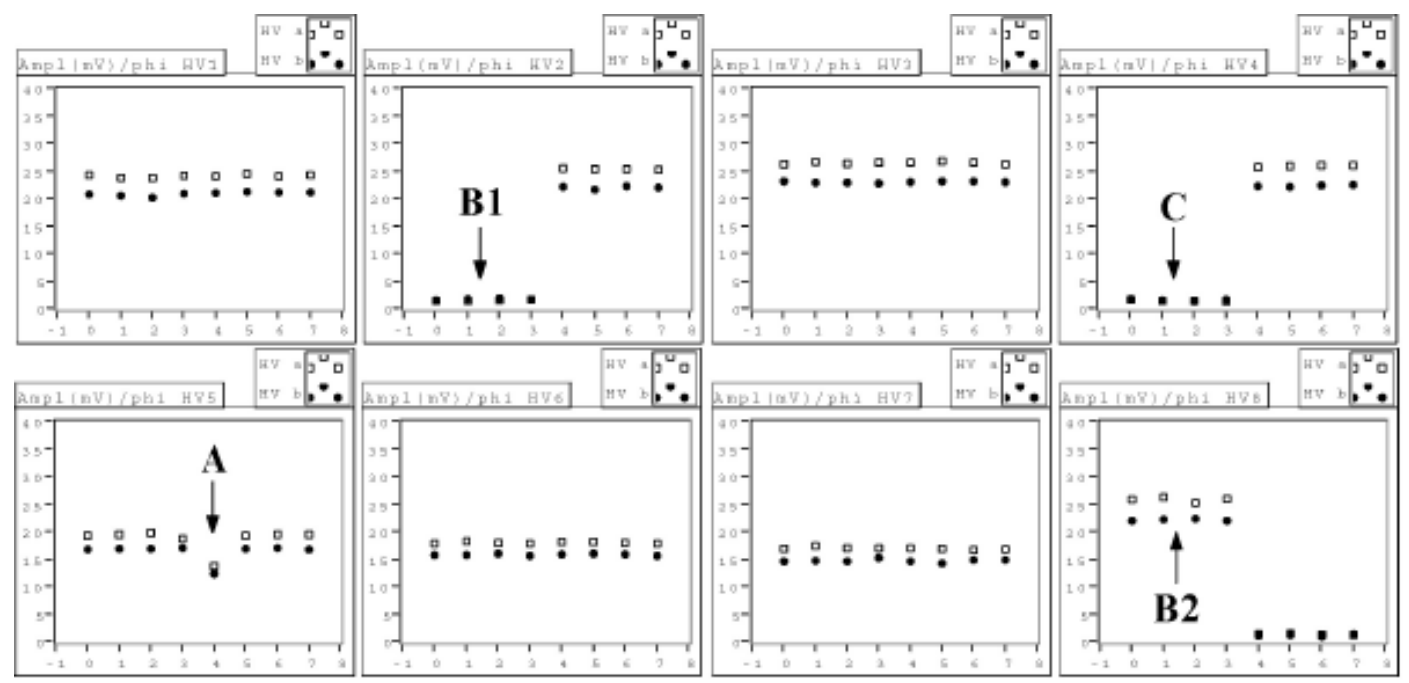

Fig. 22. Result of the TBF continuity test as a function of $\phi$ (horizontal axis) for 7 regions in $\eta$. The open squares (HV a) and full circles (HV b) correspond to the 2 high voltage lines supplying the same zone. The plots "phi HV2" and "phi HV4" correspond to sectors where the high voltage buses have been cut in two in $\phi$ (arrows B1 and C). In the plot "phi HV5", one electrode has been disconnected from the high voltage bus (arrow A). The missing half sectors or electrodes are supplied by spare lines, also tested, e.g. the plot "phi HV8" shows the second half of the sector shown in the plot "phi HV2" (arrow B2).

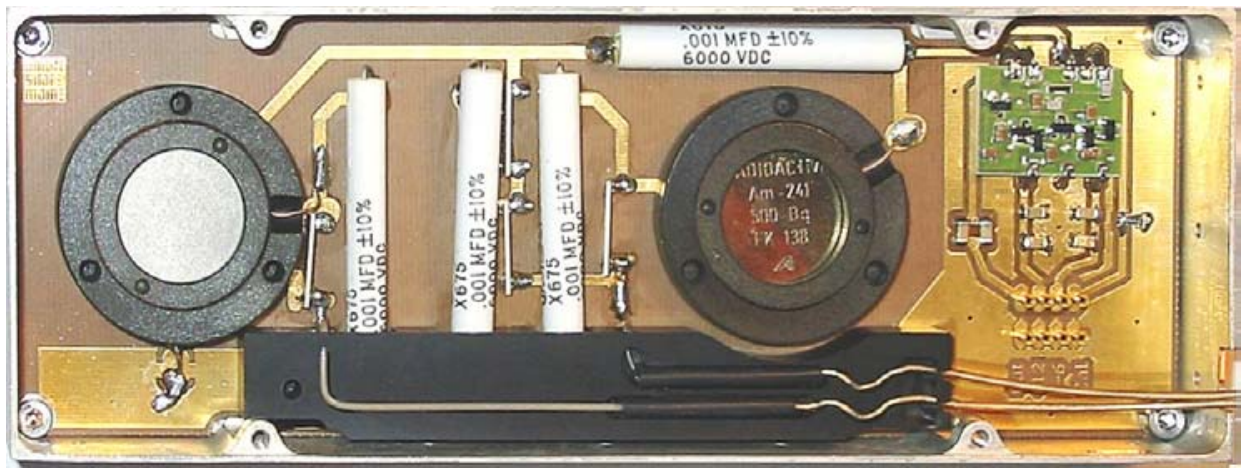

Fig. 23. The liquid argon purity monitoring device in its shielding box. 


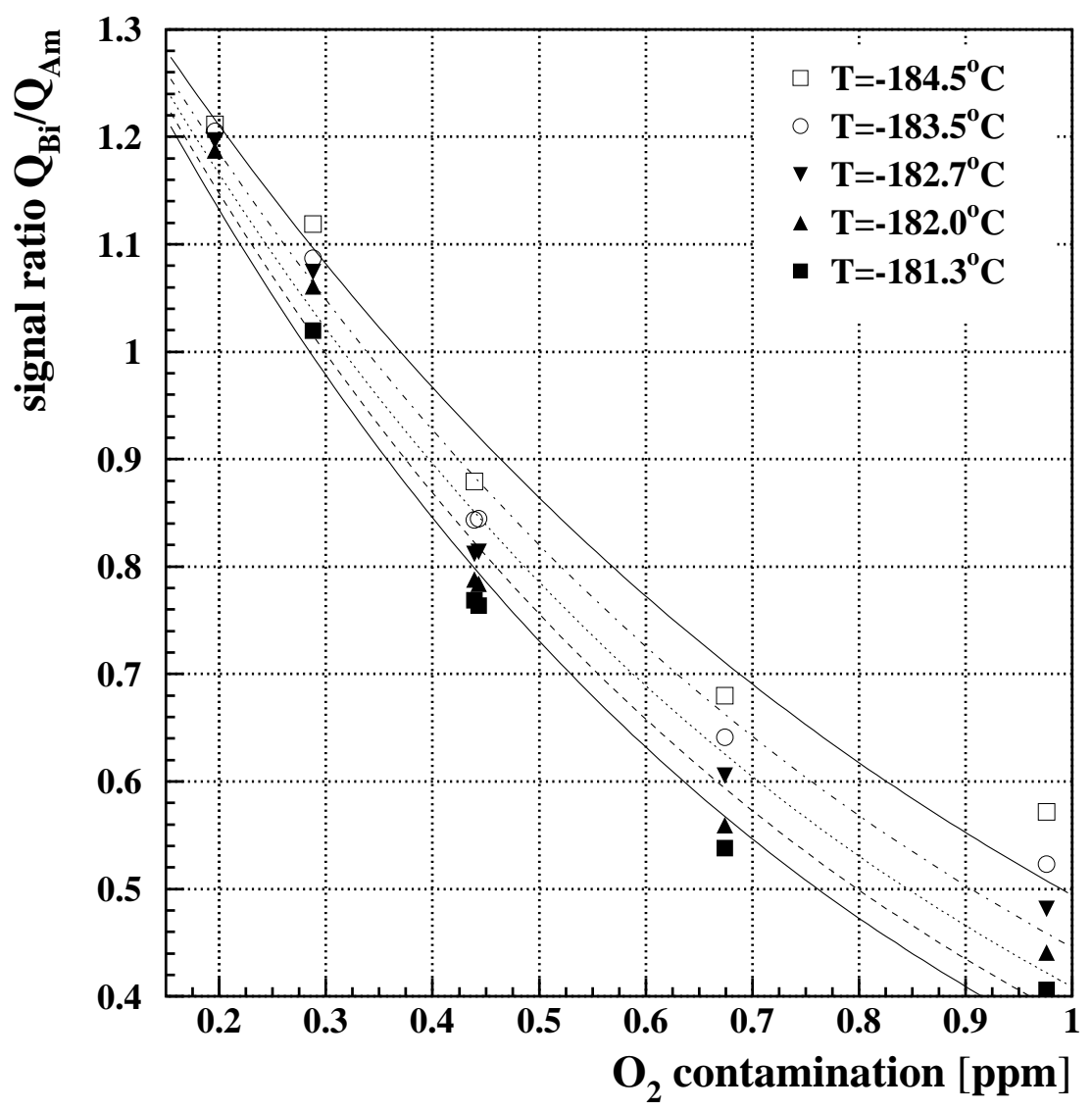

Fig. 24. Calibration of the signal ratio of the measured charges in the two cells of the purity monitors. 


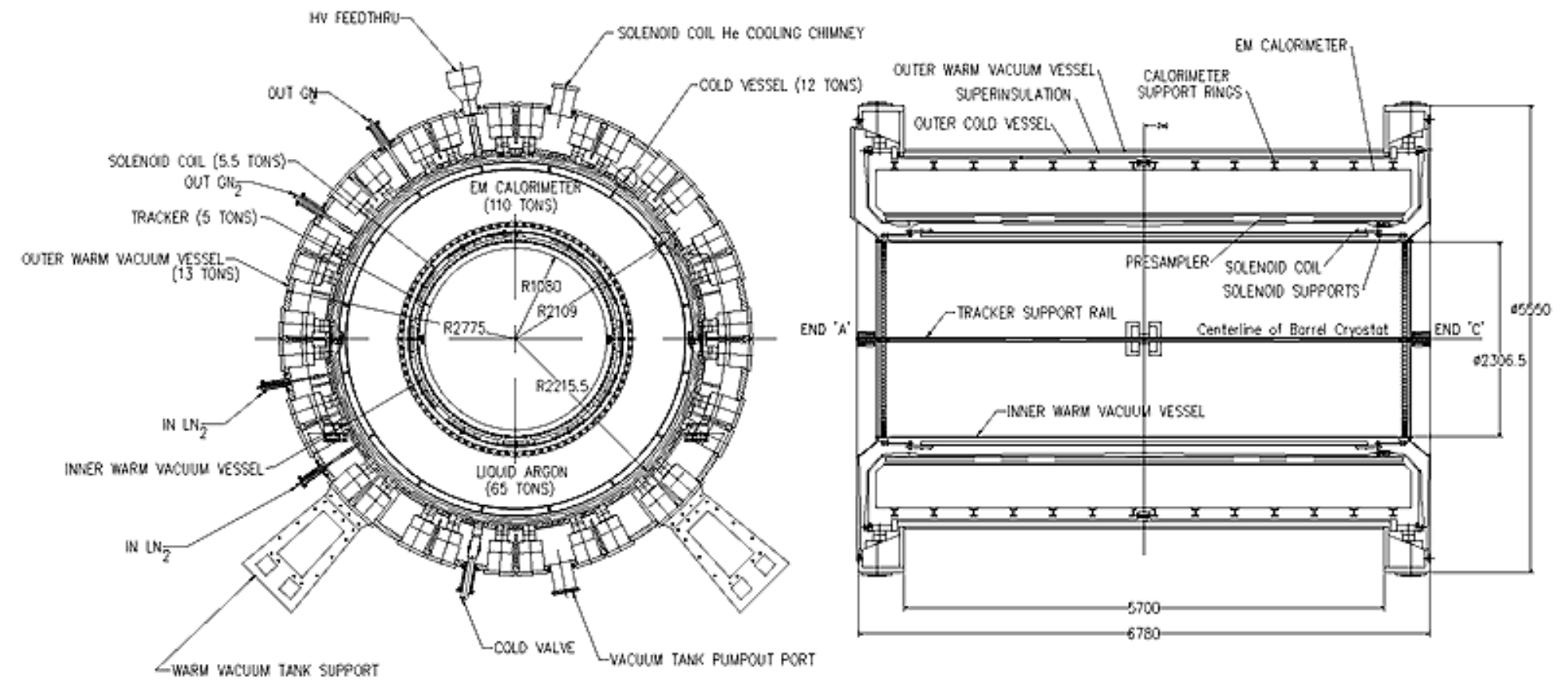

ATLAS BARREL CRYOSTAT ASSEMBLY

Fig. 25. ATLAS barrel cryostat drawing. 


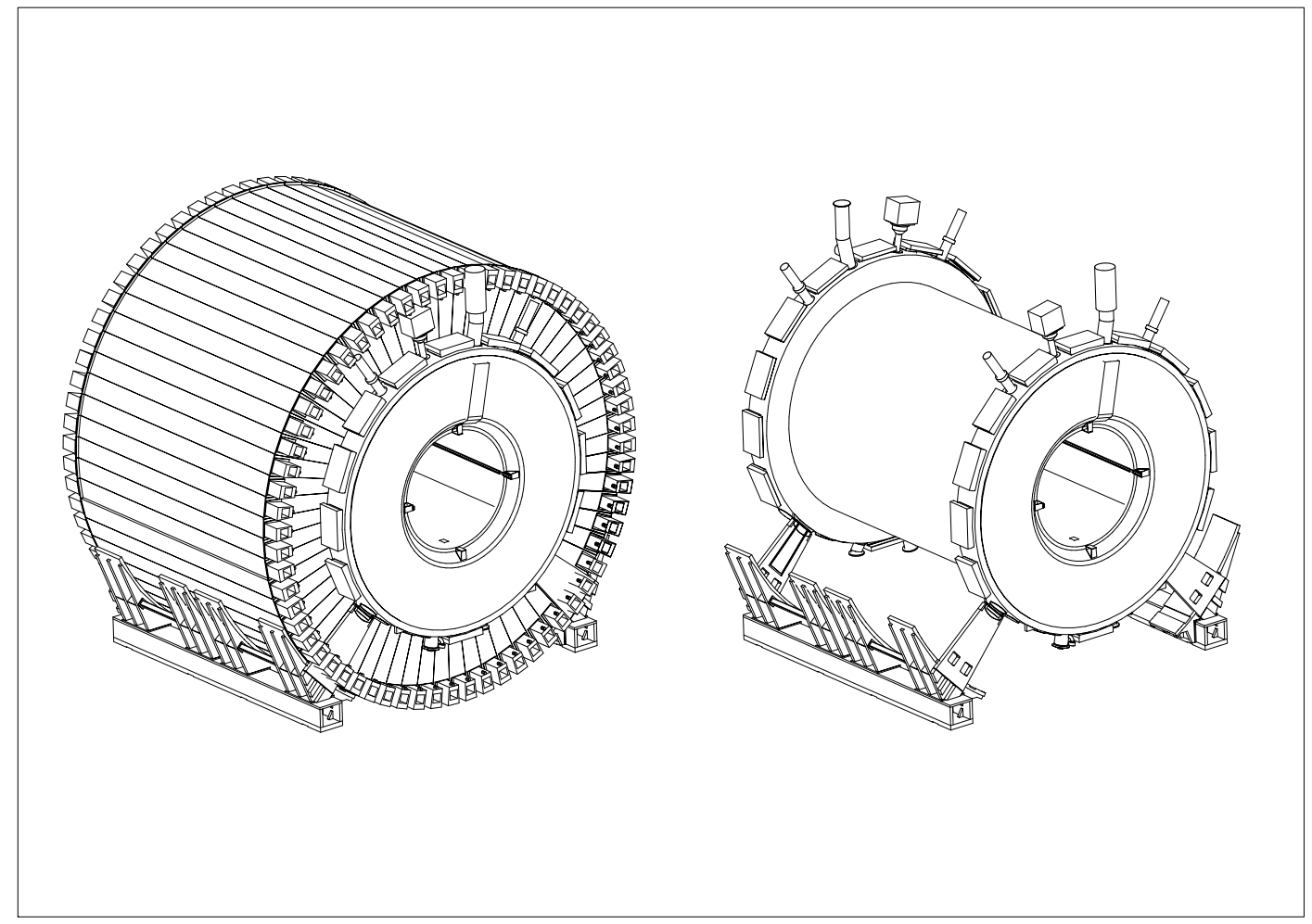

Fig. 26. Barrel Calorimeter assembly: The left drawing shows the entire barrel calorimeter assembly consisting of LAr barrel cryostat, Tile barrel and support structures. In the right drawing the Tile barrel is masked in order to illustrate better how the LAr barrel cryostat is supported. 


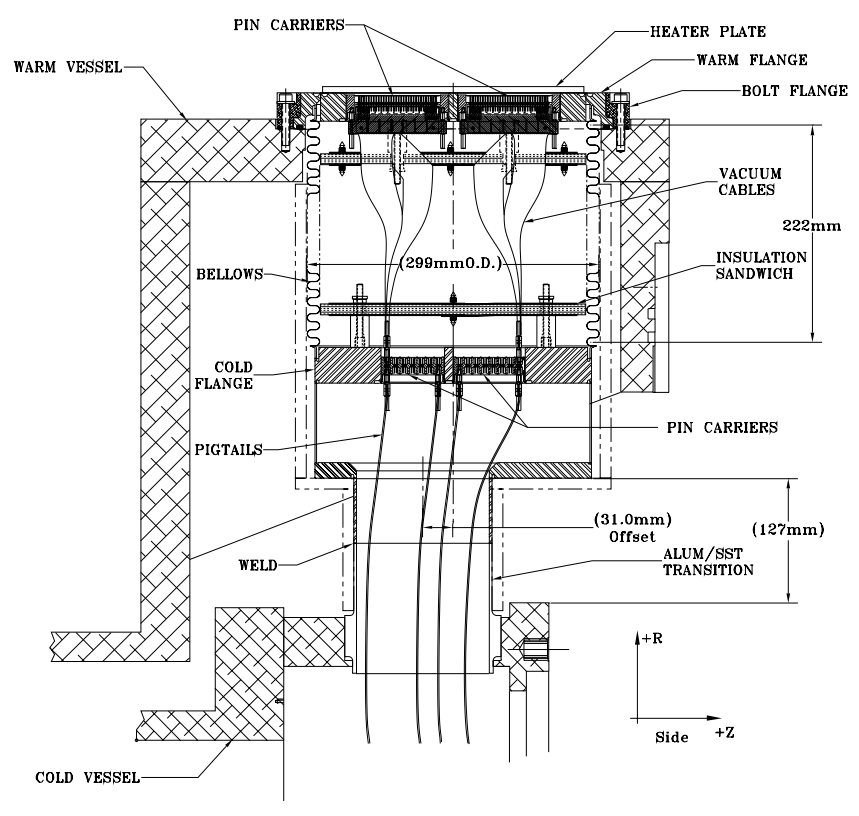

Fig. 27. An installed signal feedthrough. The figure shows the "envelope" of all 30 vacuum cables 


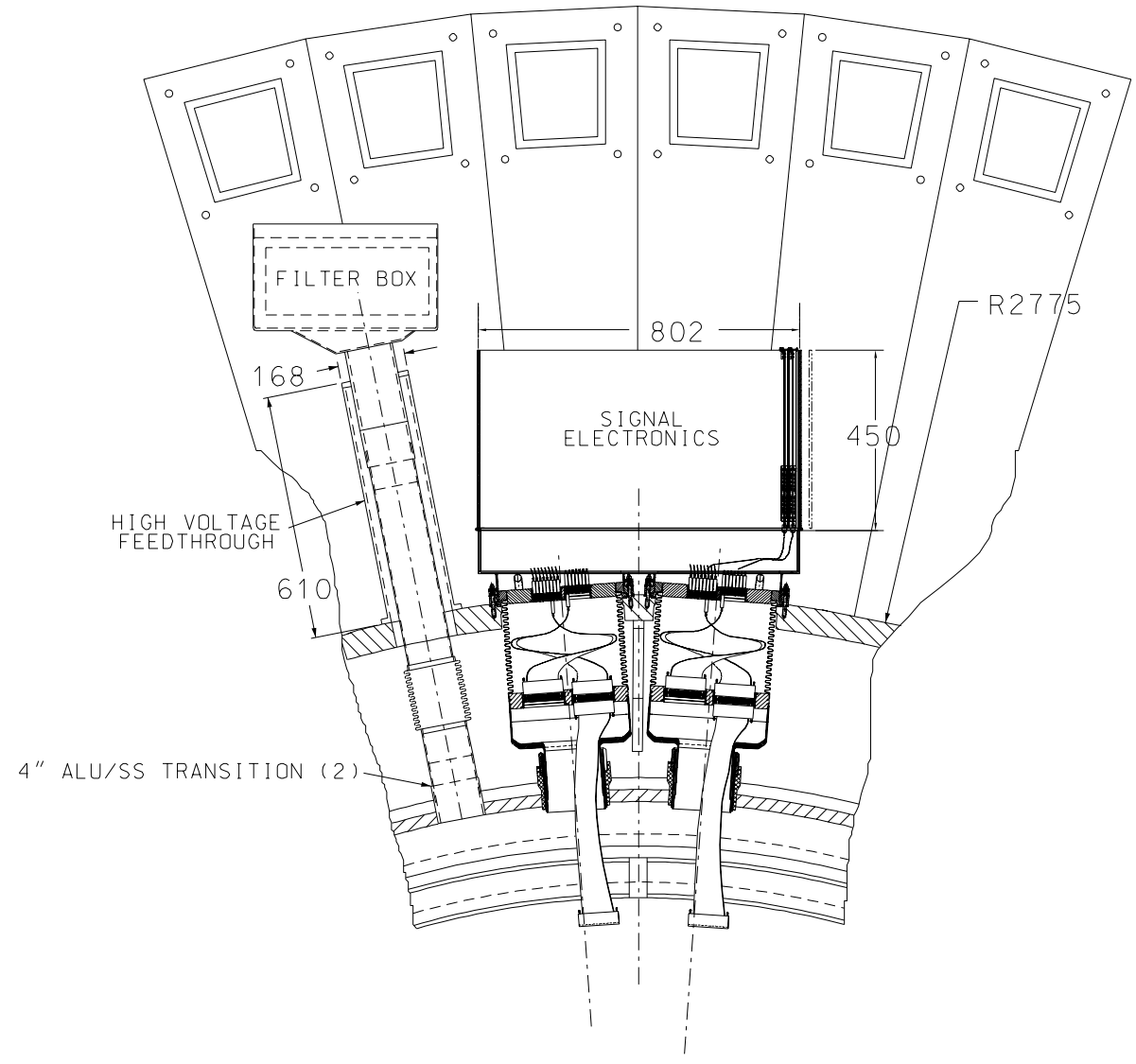

Fig. 28. A high voltage feedthrough for $840 \mathrm{HV}$ lines. The feedthrough is from air to argon gas. The filter box contains separate $\mathrm{RC}$ filters for each line. The figure shows only a representative pair of vacuum cables and one B harness in each of the two signal feedthroughs. The sectors in the background are representative of Tile Calorimeter sectors. 


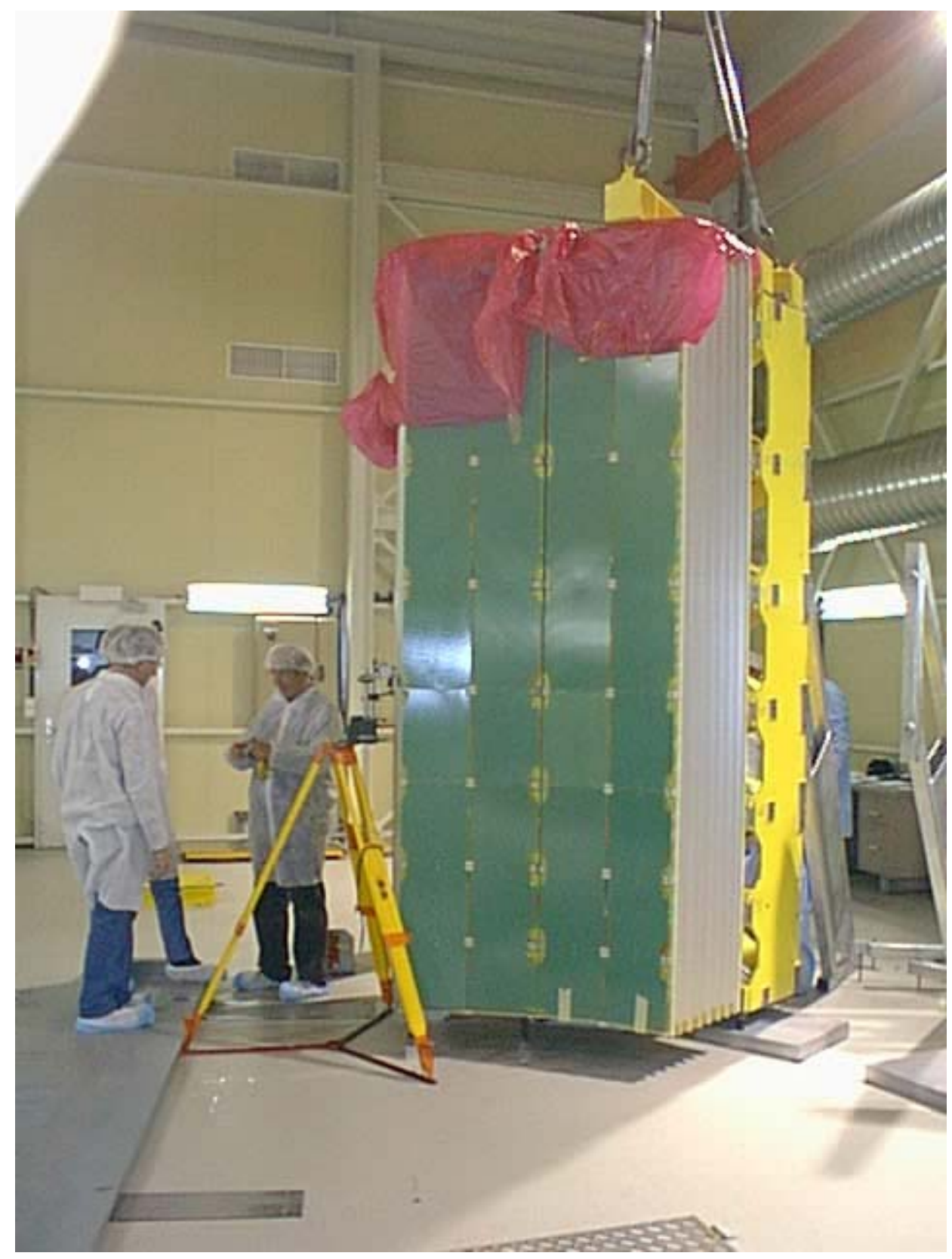

Fig. 29. The first 2 modules are joined together. 


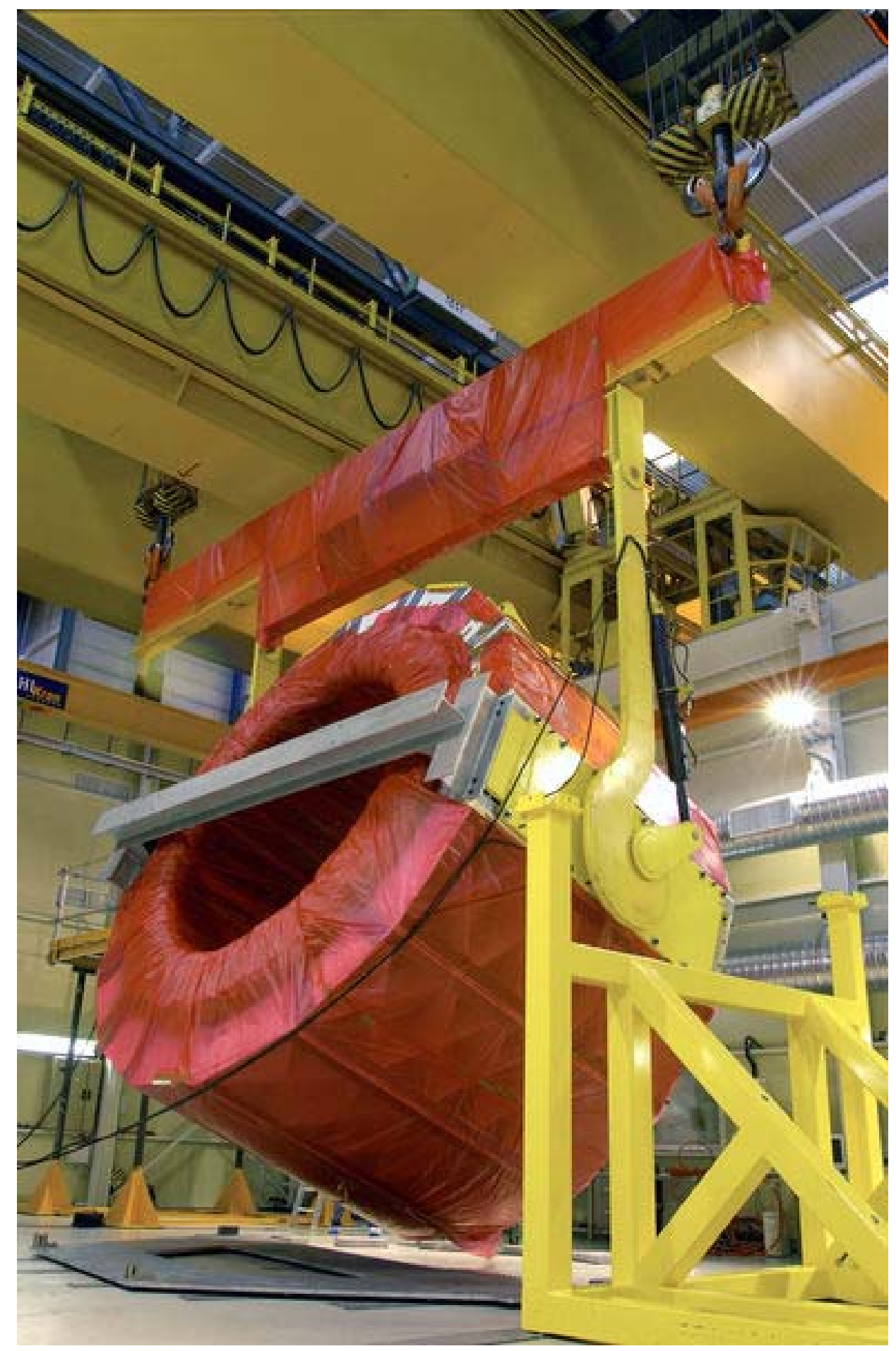

Fig. 30. Rotation of a half barrel from vertical axis to horizontal axis. 


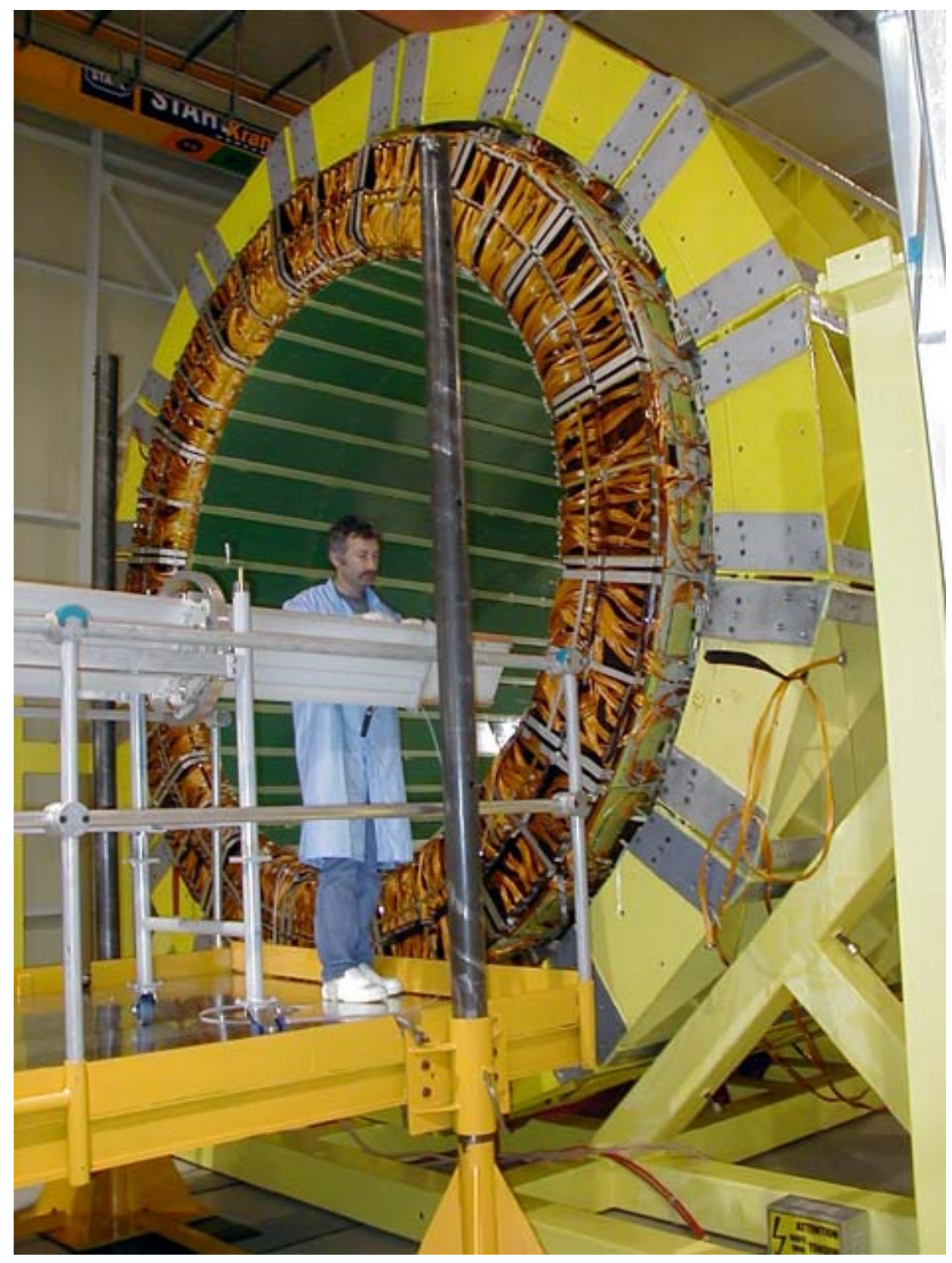

Fig. 31. Insertion of a barrel presampler sector. 


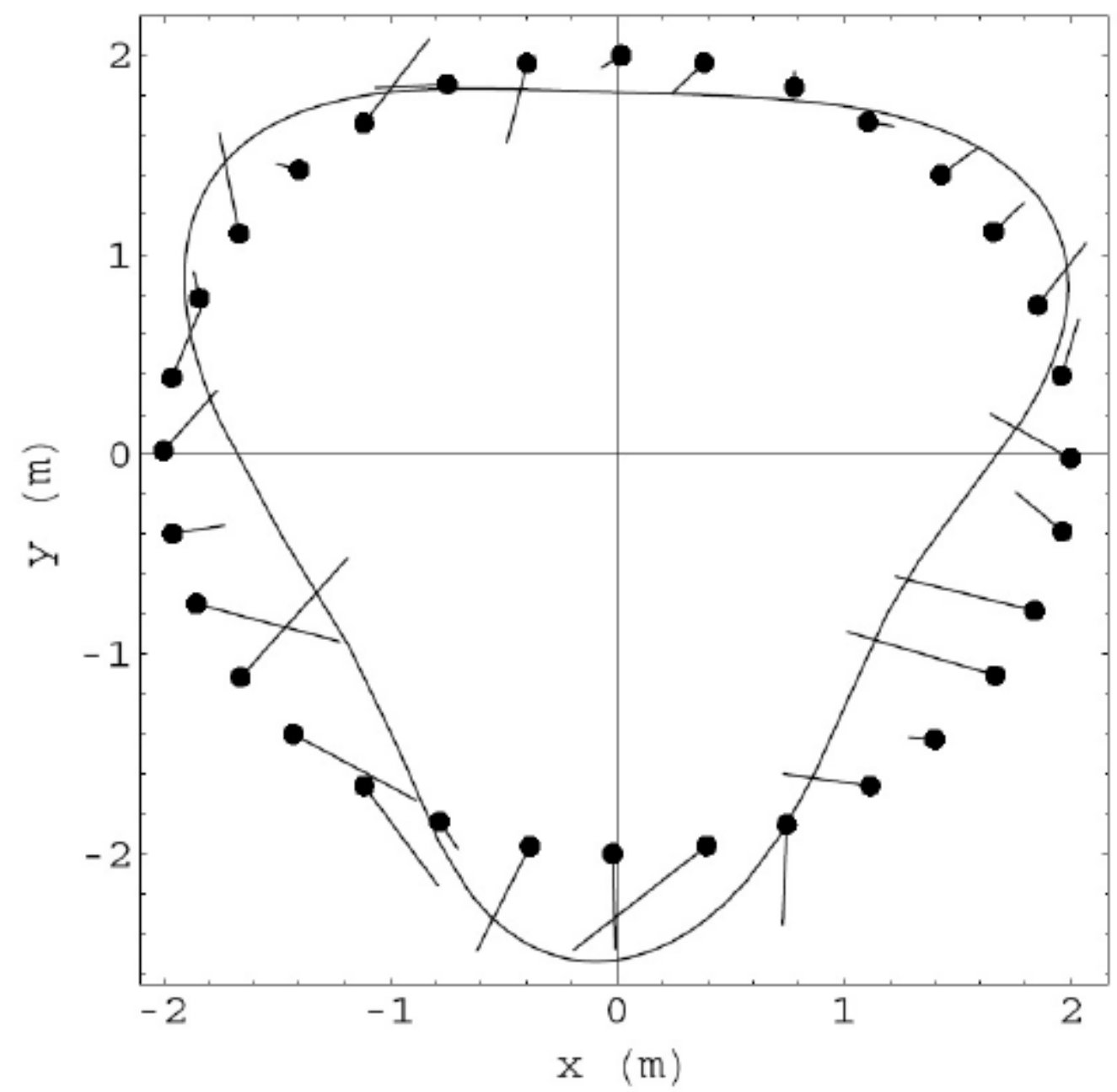

Fig. 32. Deformation of one half barrel measured at the outer survey marks. The so-called pear shape arises from the gravitational sag while the support rails remain fixed in the horizontal plane. The dots indicate the ideal, i.e. circular position of the survey marks. The dashes show the measured deviations with respect to the ideal positions - the results are exaggerated by a factor 200 . The continuous line is a fit of the half barrel shape. 

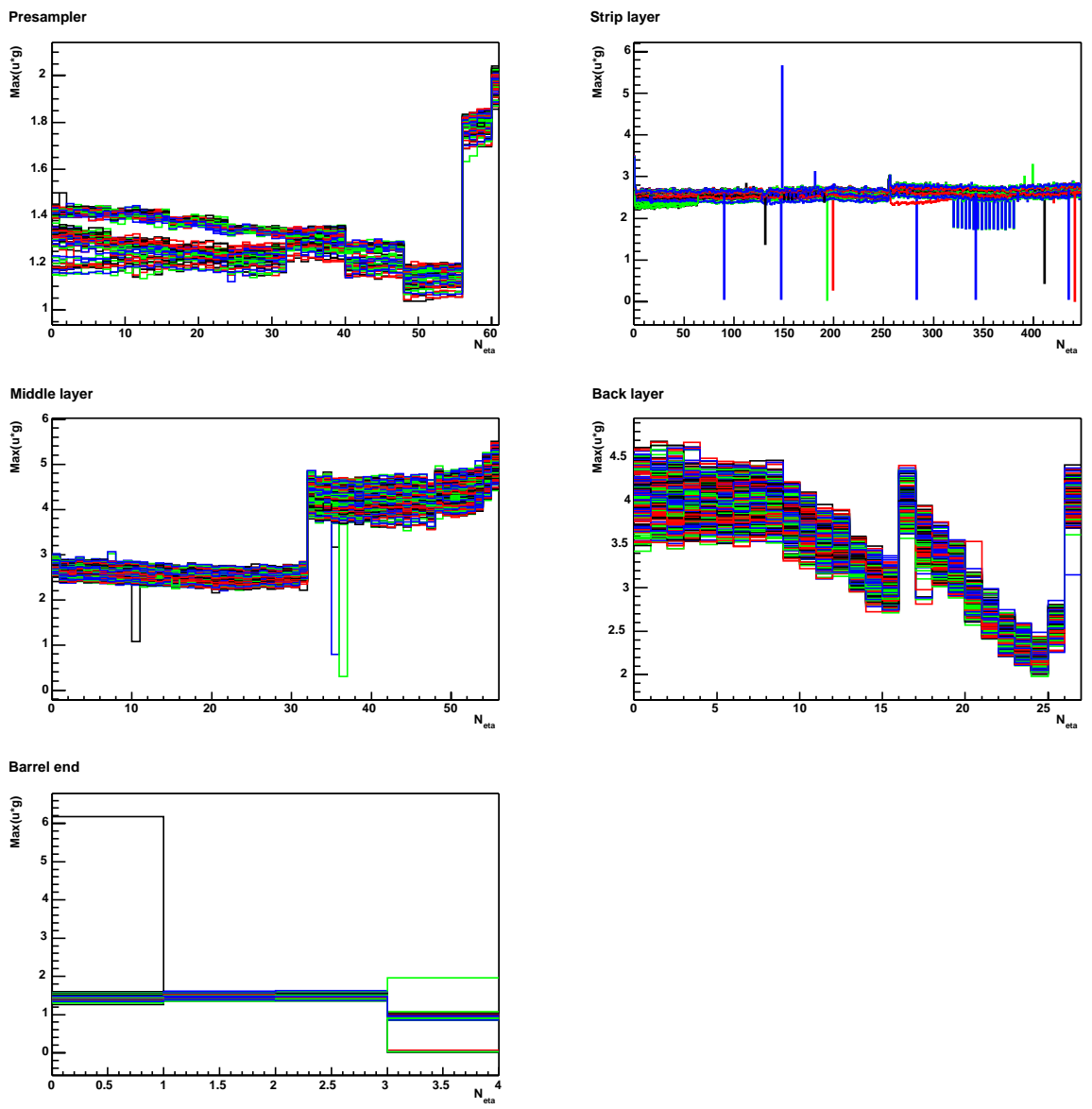

Fig. 33. Maximum, after shaping, of the test signal as a function of the $\eta$ number for all the $\phi$ lines of the whole calorimeter. The 5 plots correspond to the presampler (7808 channels), the three calorimeter layers (57216, 28672 and 13824 channels) and the barrel-end (2048 channels). Cells with problems (e.g. no signal) can be clearly identified. 


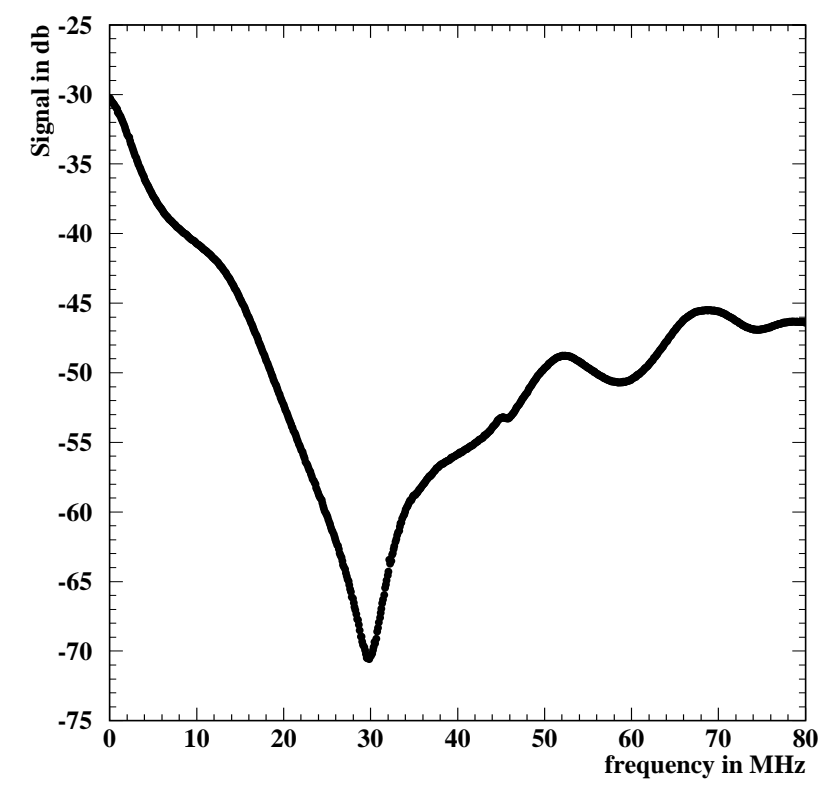

Fig. 34. Transfer function signal coming out of the network analyser, in dB.

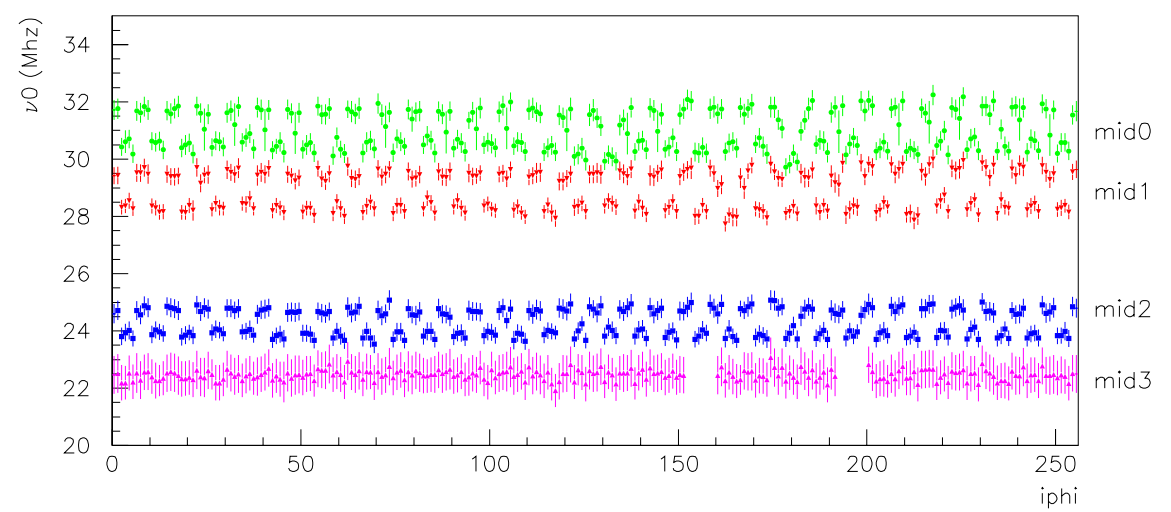

Fig. 35. Resonance frequency ( $\nu_{0}$ in $\mathrm{MHz}$ ) per $\phi$ unit, for four $\eta$ regions of the middle compartment of the first half barrel: mid0 in green $(\eta<0.4)$, mid1 in red $(0.4<\eta<0.8)$, mid2 in blue $(0.8<\eta<1.2)$ and mid3 in purple $(1.2<\eta<1.4)$. For clarity, in mid3 region, $\nu 0-2 \mathrm{MHz}$ instead of $\nu_{0}$ is plotted. 

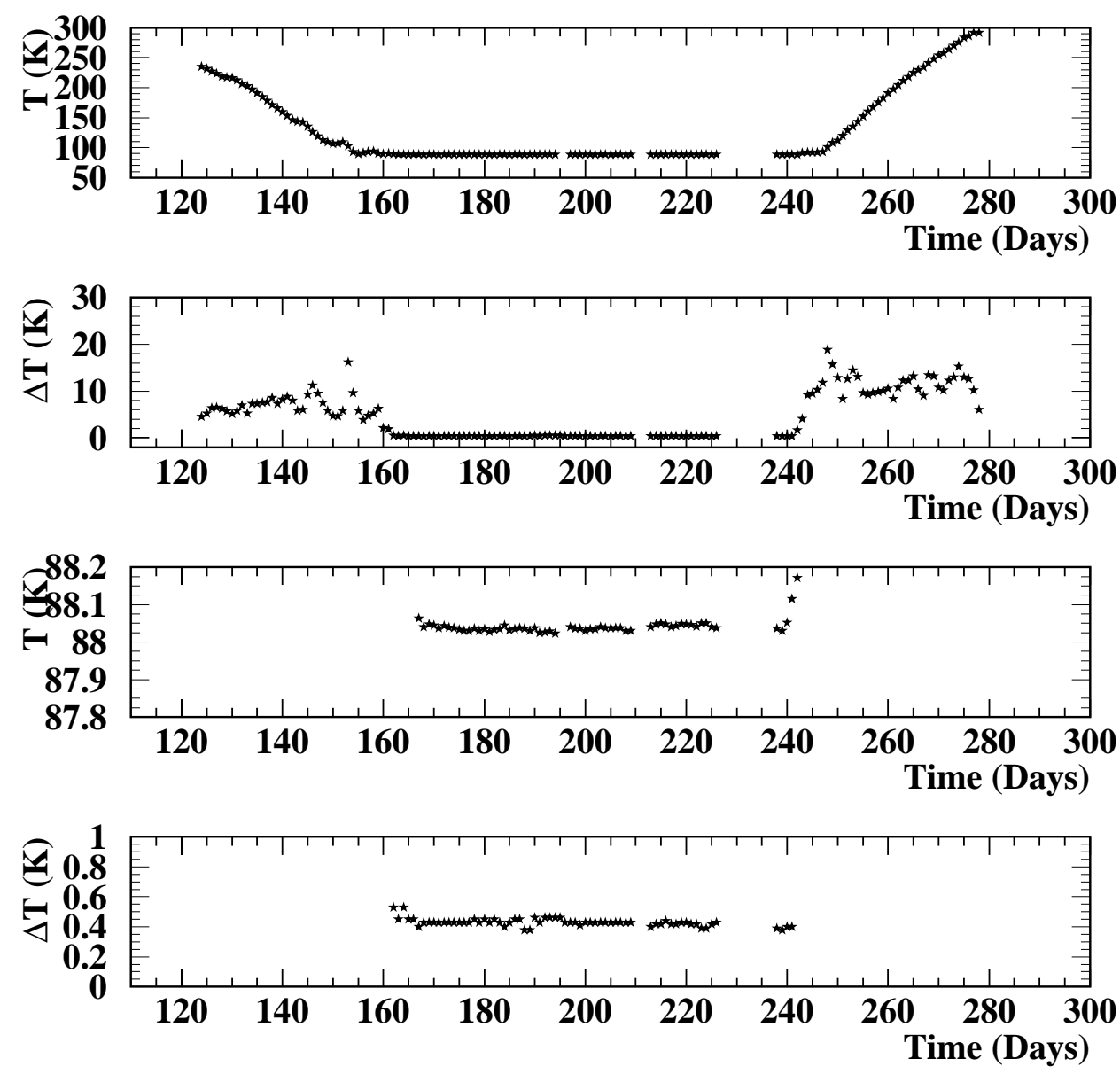

Fig. 36. The 2 upper plots show the temperature variation and the temperature difference between the warmest and coldest points inside the barrel for the whole cooling period whereas the 2 lower plots show only the part where the cryostat was filled with liquid argon (and with an enlarged scale). 


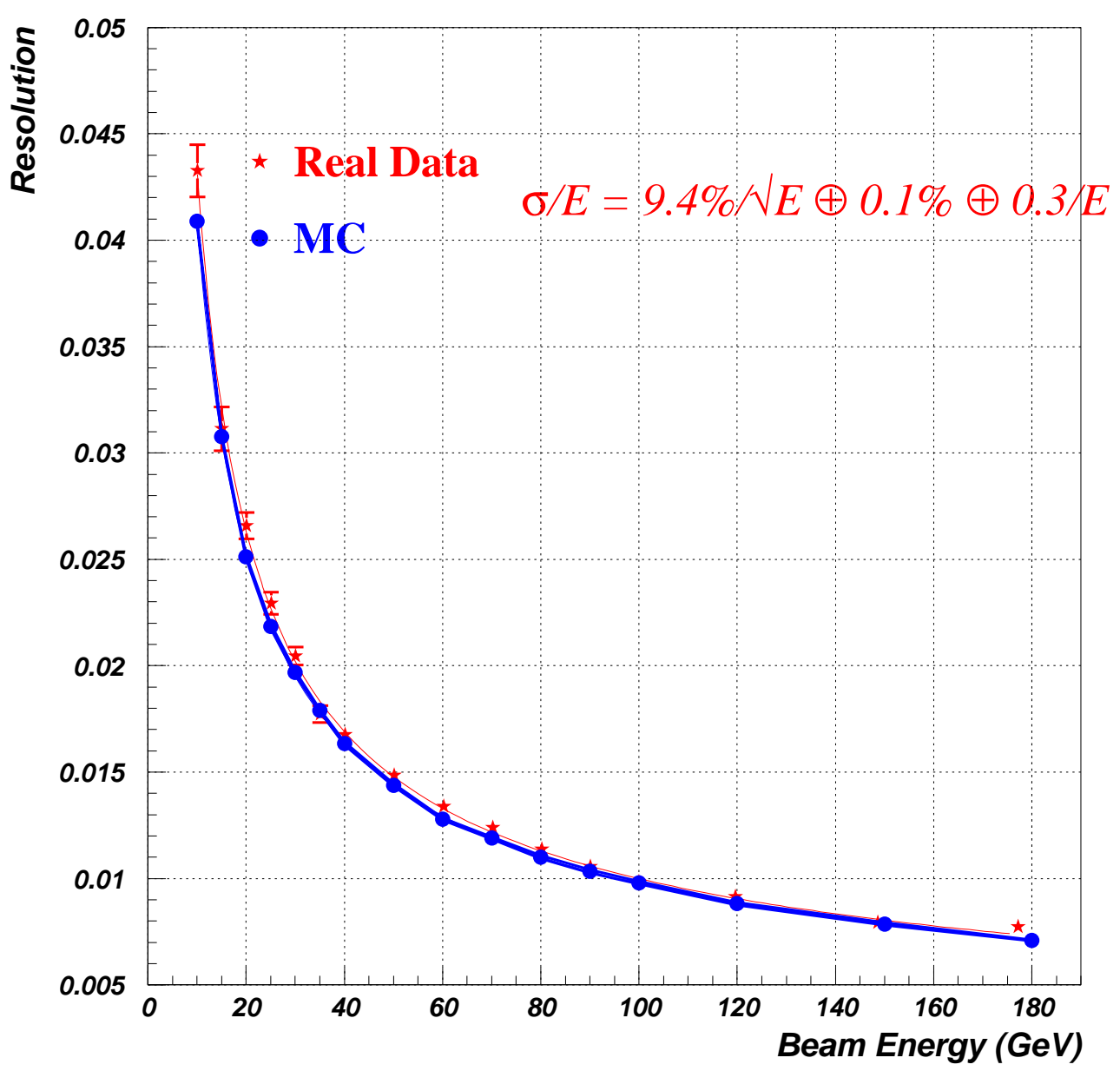

Fig. 37. Example of energy resolution. 


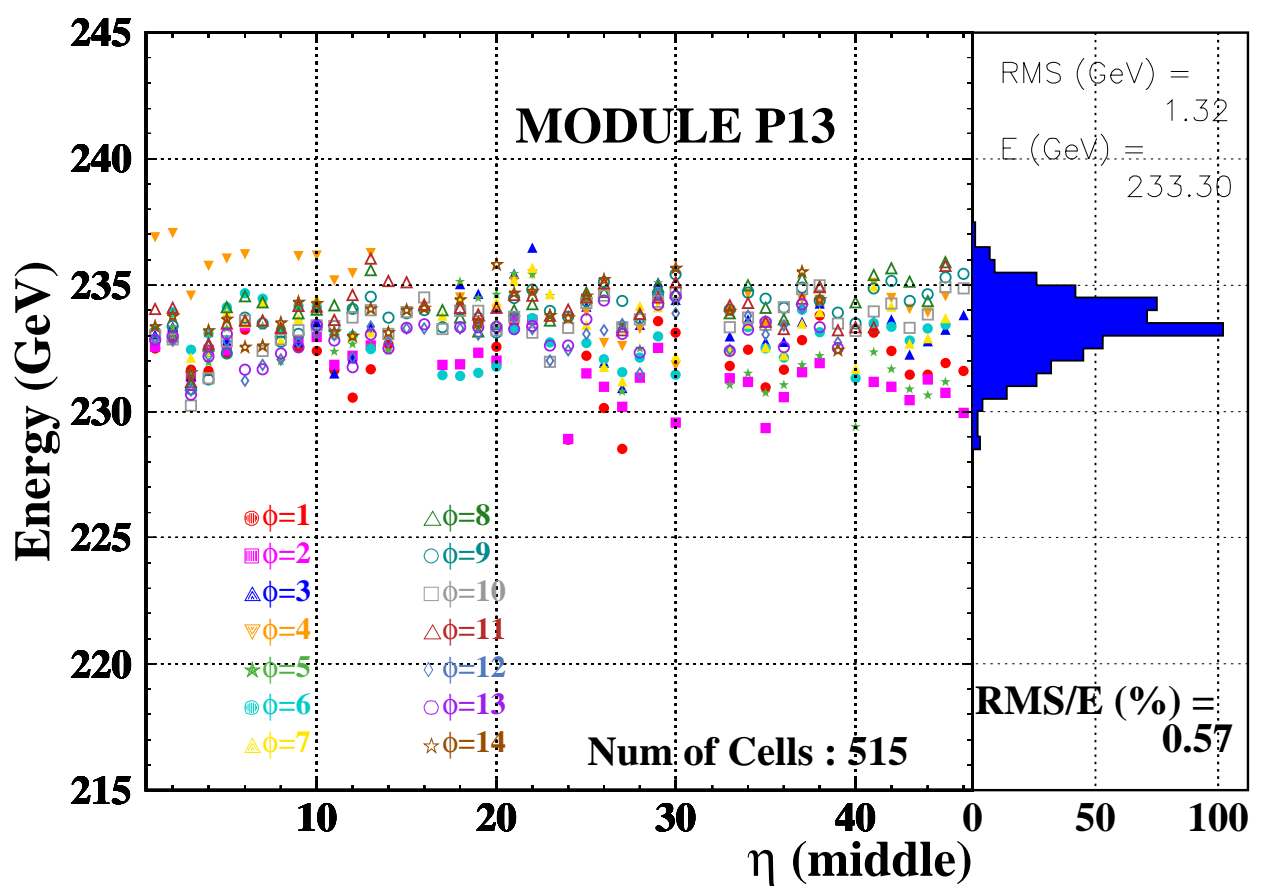

Fig. 38. Uniformity in module P13: a uniformity of $0.57 \%$ has been obtained on a total of 515 middle cells. The electron shower is not fully contained in the chosen cluster size. The seen fraction (95.2\%) of electron energy is in agreement with Monte Carlo simulation. 

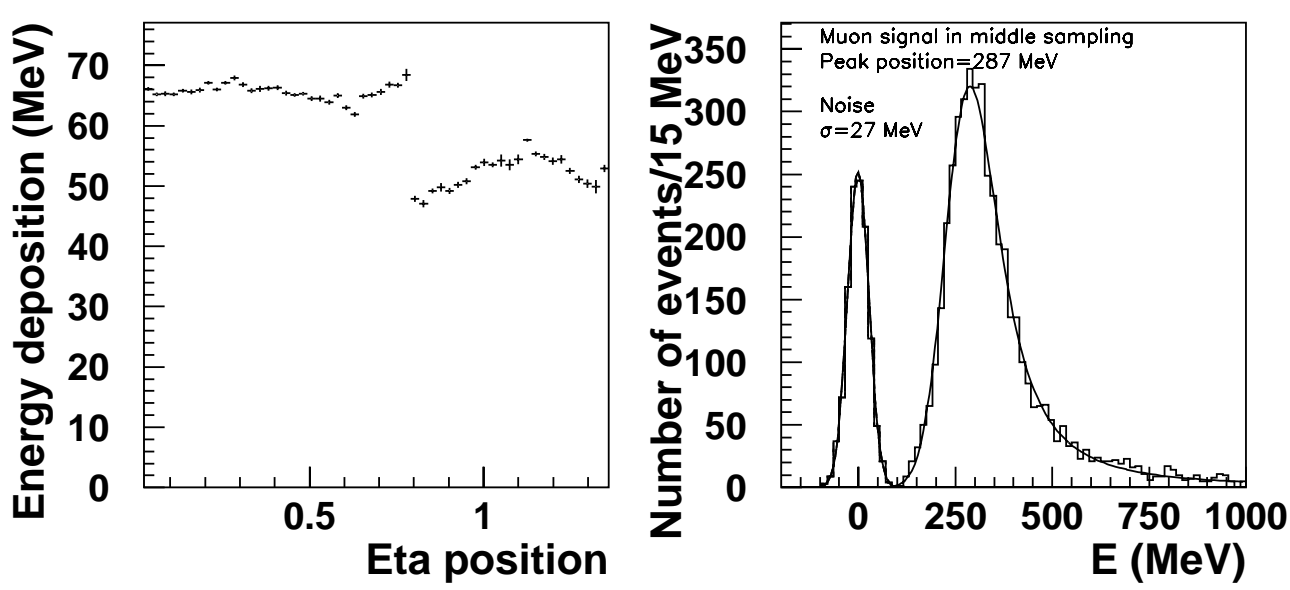

Fig. 39. The left plot shows the average muon deposit (in $\mathrm{MeV}$ ) in the front compartment as a function of $\eta$. The error bar is the error with which the Landau fit determines the average deposit. The right plot shows the muon signal in a middle cell $(\eta=0.6)$. The ratio of the mean signal over the noise RMS is 10.6 in this cell. 